\title{
Minimizing thermal conductivity in laser deposited multilayers
}

\author{
Dissertation
}

zur Erlangung des mathematisch-naturwissenschaftlichen Doktorgrades

"'Doctor rerum naturalium"”

der Georg-August-Universität Göttingen

im Promotionsprogramm ProPhys

der Georg-August University School of Science (GAUSS)

vorgelegt von

Florian Döring

aus Heilbad Heiligenstadt

Göttingen, 2016 


\section{Betreuungsausschuss}

Prof. Dr. Hans-Ulrich Krebs, Institut für Materialphysik, Georg-August-Universität Göttingen Prof. Dr. Hans Hofsäss, II. Physikalisches Institut, Georg-August-Universität Göttingen

\section{Mitglieder der Prüfungskommission}

Referent: Prof. Dr. Hans-Ulrich Krebs, Institut für Materialphysik, Georg-August-Universität Göttingen

Korreferent: Prof. Dr. Hans Hofsäss, II. Physikalisches Institut, Georg-August-Universität Göttingen

\section{Weitere Mitglieder der Prüfungskommission}

Prof. Dr. Cynthia Volkert,

Institut für Materialphysik, Georg-August-Universität Göttingen

Prof. Dr. Philipp Vana, Institut für Physikalische Chemie, Georg-August-Universität Göttingen

Prof. Dr. Michael Seibt,

IV. Physikalisches Institut, Georg-August-Universität Göttingen

Prof. Dr. Vasily Moshnyaga,

I. Physikalisches Institut, Georg-August-Universität Göttingen

\section{Tag der mündlichen Prüfung:}




\section{Contents}

1. Introduction $\quad 1$

1.1. General Introduction . . . . . . . . . . . . . . . . . 1

1.2. Thesis outline ...................... 5

2. Scientific Background 7

2.1. Heat transfer and scattering . . . . . . . . . . . . . . . . 7

2.2. Multilayers and superlattices ... . . . . . . . . . . . . . . . . 13

2.3. Heat transport models . . . . . . . . . . . . . . . . . . . 17

2.3.1. Diffusive Transport . . . . . . . . . . . . . . . . 19

2.3.2. Boundary Resistance . . . . . . . . . . . . . . . 23

2.3.3. Ballistic Transport . . . . . . . . . . . . . . . 24

2.4. Transient thermoreflectometry . . . . . . . . . . . . . . 29

3. Phonon localization in ultrathin layered structures 37

4. Confinement of phonon propagation in laser deposited Tungsten/Polycarbonate multilayers

5. Thermal conductivity measurement by transient thermoreflectometry using high-fluence excimer laser pulses at $248 \mathrm{~nm}$ wavelength

6. Minimized thermal conductivity in highly stable thermal barrier $\mathrm{W} / \mathrm{ZrO}_{2}$ multilayers

7. Further unpublished results

7.1. Thermal conductivity of atomic layer deposited thin films of $\mathrm{Pt}$ and

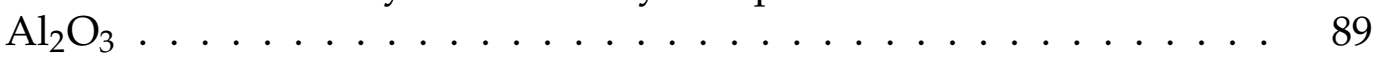

7.2. TTR-measurements on the first PAzoPMA polymer . . . . . . . . . 92

7.3. Thermal conductivity measurements on W/PC multilayers . . . . 93

$\begin{array}{lr}\text { 8. Conclusion } & 97\end{array}$

$\begin{array}{ll}\text { Bibliography } & 103\end{array}$

$\begin{array}{ll}\text { A. Author contributions } & 125\end{array}$

$\begin{array}{ll}\text { B. List of Publications } & 127\end{array}$ 



\section{Introduction}

\subsection{General Introduction}

Heat, light and electricity are three foundation pillars not only of physics, but also of our daily lives. Therefore, these have been studied by scientists for centuries and an always better understanding of the underlying exchange particles, phonons, photons and electrons, was gained. In physics, core disciplines opened up in the fields of phononics, optics and electronics [1-3]. While the latter had great technological impact on our society, the field of phononics is rather new and currently seen in the ambitious light of potentially following up on the impact of electronics almost a century ago [4].

The field of phononics deals with the propagation of mechanical waves in condensed matter [5] and is studied in different bulk materials [6], as well as nanostructures [7], special metamaterials [8,9], phononic crystals [10, 11], superlattices $[12,13]$ and multilayers $[14,15]$ with possible applications in fields of acoustics [16, 17], vibration [18], communication [19] as well as thermal storage $[20,21]$ and transport $[22,23]$. Especially the aspects of thermophysics are of great interest today, since by far most of the energy we consume results from thermal processes $[24,25]$. Thus, a better understanding of thermal processes could help with current and prevailing energy issues worldwide.

Therefore, the reduction of thermal conductivity in modern materials is an exciting question. This issue is not only important for thermal insulation [26] and storage [27] and could then find applications in cryogenics [28], aerospace industry [29] or damping [30], but also for energy conversion for instance in the field of thermoelectrics [31], solid state refrigeration [32] or as thermal barrier coatings [33] that allow thermal engines, like gas turbines, to run at higher temperatures and consequently with enhanced efficiencies.

In order to answer this question, one has to study thermal transport processes and thus the propagation of phonons. Phonons are elemental excitations of a quantized elastic lattice and belong to the family of bosonic quasi-particles [34]. They can be 
best understood in terms of lattice vibrations in a solid state object that carry wave energy through interatomic forces. In terms of long wavelengths this is known as sound, but as the frequencies get higher and therefore the wavelengths shorten, heat is conducted by lattice waves [4]. For minimizing thermal conductivity, it is essential to hinder phonon propagation through the material. This can be achieved by different approaches and will only be described briefly here in the introduction since more details will follow in chapter 2. The thermal conductivity scales with the velocity and the mean free path of the phonons. Since those values are comparably high in a periodic lattice with strong atomic bonds, crystalline materials have a much higher thermal conductivity than amorphous ones, which can be seen in Figure 1.1.

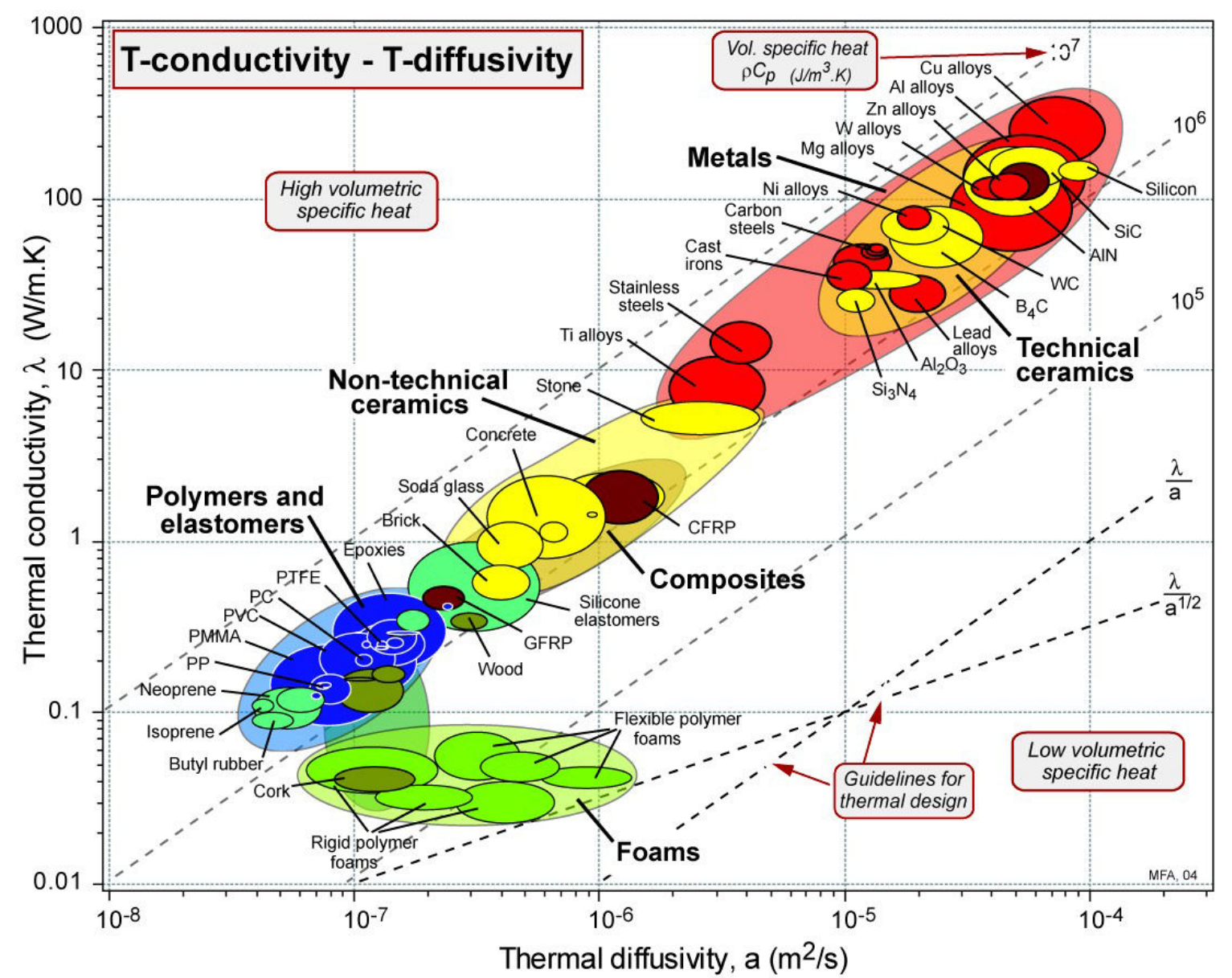

Figure 1.1.: Schematic comparison of typical thermal conductivities of different material classes. Note that thermal conductivity can be ordered from metals over crystalline materials down to amorphous materials, polymers and foams [35]. (Figure taken from [36])

In amorphous materials, phonon propagation is hindered due to the absence of the 
ordered lattice making them better thermal insulators [37]. But heat transport can be suppressed further by an increase of phonon scattering and thus a reduction of mean free path [38]. Phonon scattering is a typical process in heat conduction, but the number of scattering centers can be increased artificially, for instance by increasing the amount of boundaries which have to be passed. This is the case for thin film multilayers, where stacking of nanoscale individual layers allows for a great interface density [39]. At each interface, phonons can be scattered or even reflected, which can lead to interference and thus, to a change in phonon dispersion and the formation of band gaps [40]. At this point, phonon modes can be confined and localized so that they do not contribute to thermal transport anymore. Furthermore, a reduction in phonon group velocity and enhanced scattering rates can be seen for some frequencies [41-43]. In this way, a reduction of phonon mean free path and group velocity can be achieved, which results at the same time in a minimization of thermal conductivity $[44,45]$.

For fabricating such thin film multilayers, a variety of methods exist, for instance sputtering, atomic layer deposition, molecular beam epitaxy or pulsed laser deposition [46]. From all these methods, the pulsed laser deposition technique is outstanding, because it allows not only the fabrication of nanoscale thin films and multilayers with high quality, but is beyond that a very versatile method [47-52]. This means, it enables preparation of multilayers consisting of very different components such as metals [53], semiconductors [54], oxides [55] and even polymers [56], because almost every solid material can be pulsed laser deposited. The advantage for phonon blocking materials lies in the ability of the method to combine materials with very different properties, such as hard and soft or heavy and light materials, for instance metals and polymers [57]. Such multilayers have the potential for a high number of interfaces with very different material properties at each side of the interface. Since the interface resistance gets stronger with a greater difference in density and sound velocity at each side [58-60], pulsed laser deposition enables to fabricate multilayers with a high density of strongly resisting interfaces.

In order to get information about phononic transport in such structures, one has to measure the elastic dynamics on very short timescales [61,62]. Therefore, a close cooperation with the working groups of Henning Ulrichs from the $1^{\text {st }}$ Institute of Physics in Göttingen and Markus Münzenberg from the Institute of Physics in Greifswald has been established in order to perform fs-pump-probe measurements of the pulsed laser deposited samples. Here, an ultrashort laser pulse is split up in 
a pump and a probe beam that both hit the sample with a very short delay in time. Thereby, the sample gets excited by the pump pulse and its reflectivity dynamic is measured with the probe pulse repeatedly with different delays. In that way, a time-dependent reflectivity signal can be obtained from which information about phonon modes can be deduced.

For the measurement of thermal conductivities, different methods come into consideration, like pyrometry [63], the 3-omega technique [64], photodisplacement [65], laser flash [66] or thermoreflectance methods [67,68]. Since pulsed laser deposition allows the preparation of a great variety of thin film multilayers, the measuring method should also be highly flexible and applicable to a large group of samples with low preparation effort [69-72]. Therefore, the method of transient thermoreflectance (TTR) is prominent, which also works as a pumpprobe scheme, comparable to the phonon dynamics measurements [73]. Since thermal conductivity is a much slower, diffusive process, a measurement on the $\mu$ s timescale without the need of ultrafast lasers is applicable. This method works by heating up the sample surface with a laser pulse (this time with a pulse length in the ns regime) and utilizing at the same time a second, continuous wave laser in order to measure the reflectivity of the sample surface, which is tracked with a fast Si-photodiode and an oscilloscope. From the temporal evolution of the reflectivity after pulsed laser heating, the temperature at the surface can be obtained. Taking these measured information into account and fitting them to the solution of the heat conduction equation, one can get information about the thermal conductivity [74].

Aim of the present thesis is to fabricate high-quality, nanoscale multilayers of dissimilar materials with pulsed laser deposition and to minimize the cross-plane thermal conductivity of these structures. In order to achieve this final goal, first, the deposition of metal/polymer (mainly $\mathrm{W} /$ polycarbonate) and metal/oxide (e.g. $\mathrm{W} / \mathrm{ZrO}_{2}$ ) multilayers must be perfected and phonon transport will be studied in such structures. Secondly, a measurement method capable of obtaining information about thermal conductivities has to be installed and optimized for bulk materials and especially thin films. With this, information about heat transport in nanoscale multilayers will be obtained systematically. From the combination of all those efforts, this thesis could be developed, which shall be outlined in the following. 


\subsection{Thesis outline}

Starting point of this work is the study of phononic properties in simple bilayers of $\mathrm{Cu}$ and polycarbonate (PC), where localization of phonon modes shall be investigated. Going to more complex multilayer systems with different acoustic mismatch as well as different multilayer periodicity, band structures have to be considered for phonon transport. Here, phonon blocking could become more powerful with increasing acoustic mismatch because of the interface contributions, which shall be investigated. This work on bilayers as well as on first multilayers is described in chapter 3 [75].

From the work in this paper, the question arises how phonons can be confined more efficiently. Since nanostructured metal/polymer multilayers provide a strong resistance to phonon propagation, $\mathrm{W} / \mathrm{PC}$ are chosen for further studies, meaning that the pulsed laser deposition setup must be optimized in order to fabricate multilayers of the hard and heavy material $\mathrm{W}$ together with the soft and lightweight polymer polycarbonate. Such worldwide unique structures are investigated in chapter 4 pursuing the question whether periodic W/PC multilayers are potent for phonon confinement and if one can increase the potential of this material combination by fabricating aperiodic multilayers that distort the periodic order [76].

For measuring thermal conductivities, the long-known principle of TTR seems promising for implementation because of its flexibility and low sample preparation effort. To set up this method, an excimer laser with $248 \mathrm{~nm}$ wavelength and $20 \mathrm{~ns}$ pulse duration was available, which has not been used for such measurements before and provides a novel approach for implementation of the TTR principle. This special TTR method had to be established, understood and optimized also for thin film multilayers. This is described together with an analytical solution of the heat equation, which is necessary in order to get physical data from the measurement curves in chapter 5 [77].

The final application of this method on laser deposited multilayers consisting of the high melting materials $\mathrm{W}$ and $\mathrm{ZrO}_{2}$ is explained in chapter 6 [78]. Here, the aim to minimize thermal conductivity in multilayers was directly tackled. By combining the prominent thermal barrier material $\mathrm{ZrO}_{2}$ with ultra-thin blocking layers of $\mathrm{W}$, interface resistances should reduce the overall thermal conductivity significantly. Moreover, the thermal stability of those multilayers has been studied, since a material combination with a high thermal stability and a low thermal conductivity 
could become an interesting candidate for future thermal barrier coating materials. Finally, chapter 7, which is an addendum of yet unpublished results to the four published papers, describes three selected projects, where thermal conductivity measurements with the TTR method are performed on a new kind of polymer as well as on thin films consisting of $\mathrm{Pt} / \mathrm{Al}_{2} \mathrm{O}_{3}$ and last but not least on W/PC multilayers. Those measurements on $\mathrm{W} / \mathrm{PC}$ multilayers represent an additional highlight since these nanostructures are extremely difficult to fabricate and measurements on them proved challenging due to the risk of destroying the PC. Nonetheless, great efforts were made to fabricate and measure them since they should show an ultra-low thermal conductivity.

All in all, this thesis demonstrates new and original research work in the field of phononics and thermal conductivity manipulation. It investigates the minimization of thermal conductivity in pulsed laser deposited multilayers and contributes to fundamental understanding of phononic thermal transport on the nanoscale. 


\section{Scientific Background}

The nature of heat and thereby the thermal conductivity has been studied by physicists for a long time. This field arose probably with Newtons law of cooling back in 1701 and found its first early climax in Fouriers analytic theory of heat in 1822 [79]. His heat diffusion equation became a powerful tool for almost two centuries and has been used in diffusion-like problems not only in physics, but for instance also in biology, earth sciences and even social sciences [80]. Over the centuries, prestigious people in physics have studied heat, like Maxwell, Lord Rayleigh, Einstein or Fermi, only to name a few. Even and still today, thermal conductivity is studied in different research groups worldwide in order to understand and manipulate this process. Since this present thesis also constitutes a small progress for thermal conductivity research, the following chapter shall be used to outline the most important background in order to understand the successional research work. Starting from the basics of heat transfer and typical scattering mechanisms, multilayers and superlattices are introduced as prominent structures in order to minimize thermal conductivity. Therein, heat can be conducted in two regimes, namely the incoherent diffusive regime, where boundary resistances at the interfaces play a significant role, as well as the coherent, or ballistic regime, where a wave-like behavior of phonons becomes important. Finally, the basic principles of the transient thermoreflectometry (TTR) setup, which has been implemented in this work, will be introduced.

\subsection{Heat transfer and scattering}

Heat transfer in solids is described by the thermal conductivity, which is a temperature dependent material property and primarily governed in terms of the well-known Fourier law [81]:

$$
\dot{Q}=-\kappa \nabla T
$$


Here, $\kappa$ is the thermal conductivity of the investigated material, that determines together with the temperature difference $\nabla T$ the local heat flux density $\dot{Q}$. The thermal flux occurs by the motion of thermal energy carriers from the hot side towards the cold side. In solids, those carrier particles are free electrons and phonons [82]. Heat flow in metals, which typically have a very high thermal conductivity, is mainly dominated by electrons, while in semiconductors and oxides phonons play the dominant role due to the lack of free electrons [83]. A first approach in order to minimize thermal conductivity should therefore be to confine heat conduction to phonons by using electrically insulating materials preventing conduction by free electrons. This could be done either by only choosing insulating materials or by multilayer combinations of metals together with insulating interlayers $[39,84,85]$. Such multilayers have the advantage that the interfaces between dissimilar materials provide additional resistance to phonon propagation, which reduces the thermal conductivity even more [86,87]. This can be deduced according to [88] for a one-dimensional heat flow. Considering a volumetric number density $n$ of carrier particles with energy $\epsilon$, which travel a certain distance $l=v \mathcal{T}$ in the time $\mathcal{T}$ with a velocity $v$ before facing a scattering event that results in a change in direction or energy transfer. This distance $l$ is called the mean free path and therefore $\mathcal{T}$, the relaxation time, is defined as the average travelling time between two scattering events [34]. Regarding the energy transfer in one direction (e.g. the z-direction), it can be explained by the difference of thermal fluxes flowing in the positive and negative z-direction:

$$
\dot{Q}_{z}=\frac{1}{2}\left(\left.\left(v_{z} n \epsilon\right)\right|_{v_{z+l}}-\left.\left(v_{z} n \epsilon\right)\right|_{v_{z-l}}\right)=-v_{z} \mathcal{T} \frac{\partial\left(v_{z} n \epsilon\right)}{\partial z}
$$

Considering now an isotropic medium, where the average velocity can be expressed as $v_{z}^{2}=v^{2} / 3$, the heat flux yields:

$$
\dot{Q}_{z}=-\frac{v^{2}}{3} \mathcal{T} \frac{\partial(n \epsilon)}{\partial z}
$$

With the definition of an internal volumetric energy $U=n \epsilon$, one can express the above equation as:

$$
\dot{Q}_{z}=-\frac{v^{2}}{3} \mathcal{T} \frac{\mathrm{d} U}{\mathrm{~d} T} \frac{\mathrm{d} T}{\mathrm{~d} z}
$$


Comparing this with equation 2.1 and considering the definition of the volumetric heat capacity $c=\frac{\mathrm{d} U}{\mathrm{~d} T}$, one gets the following expression for the thermal conductivity $\kappa$ :

$$
\kappa=\frac{1}{3} c v^{2} \mathcal{T}=\frac{1}{3} c v l .
$$

This equation defines in principle the thermal conductivity depending on the phononic properties in terms of heat capacity $c$, velocity $v$ and mean free path l.

For the final goal of minimizing thermal conductivity, three strategies exist. First, one could try to reduce the heat capacity, which typically happens at low temperatures and therefore reduces thermal conductivity in this area [89]. At room temperature however, this is a material property and cannot be changed easily. Secondly, one can attempt to reduce the phonon group velocity, which would reduce thermal conductivity. This can be achieved with nanoscale multilayers, because of the wave-like nature of phonons, and will be explained later in this chapter (see chapter 2.3.3). Finally, the most typical and most promising strategy to reduce thermal conductivity is to shorten the mean free path of the phonons [90-92].

This reduction of phonon mean free path can be done by several scattering mechanisms, which shall be explained briefly in the following paragraphs.

The most typical scattering process is the normal phonon-phonon scattering processes, where two incoming phonons create one outgoing phonon or where one incoming phonon decays in two outcoming ones with conservation of total crystal momentum. However, this alone cannot provide the reason for a finite thermal resistance [93-95]. Thus, more scattering mechanisms must exist that affect and reduce the thermal conductivity. In figure 2.1 it is shown, how the thermal conductivity is limited by such different scattering processes. In the low temperature regime, where only low frequency phonons exist, merely diffuse boundary scattering is important. The relaxation time $\mathcal{T}_{b}$ of this process can be expressed in the Casimir limit by $[90,96]$ :

$$
\mathcal{T}_{b}^{-1}=\frac{v}{L} .
$$

Here, $L$ denotes the size of the system, which is rather high for bulk materials. Thus, in the low temperature regime, thermal conductivity is governed by the 


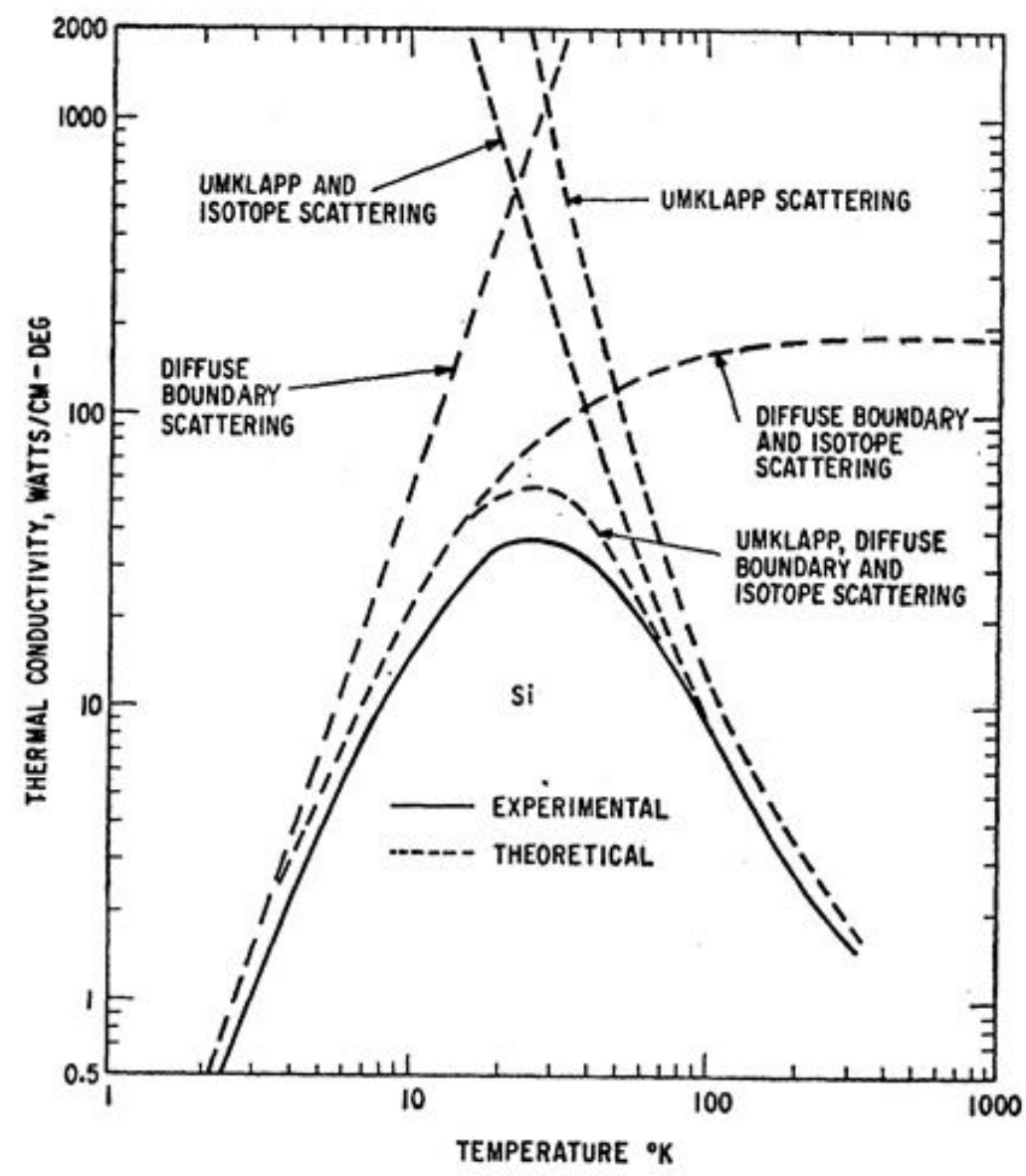

Figure 2.1.: The temperature-dependent thermal conductivity of $\mathrm{Si}$ is shown as well as calculations of different scattering mechanisms that confine this value. In the low temperature regime the diffuse boundary scattering is most important and the thermal conductivity scales with the heat capacity. However, for higher temperatures, first, isotope scattering starts to limit the thermal condcutivity before at high temperatures the Umklapp scattering becomes dominant. [90]

heat capacity, which changes with $T^{3}$ [97]. As with higher temperatures more phonon modes become occupied, other scattering mechanisms become more and more important. Now, phonons can also be scattered by impurities, like isotopes or inclusions with a mass difference from the regular lattice. For such point imperfections the relaxation times $\mathcal{T}_{i}$ for isotope scattering are $[98,99]$ :

$$
\mathcal{T}_{i}^{-1}(\omega)=\frac{3 V_{0} \Gamma \omega^{4}}{\pi v^{3}}
$$

Where $\omega$ is the phonon frequency, $V_{0}$ the average volume per atom and $\Gamma$ is a parameter that describes the strength of the impurity scattering [100]. With increasing temperature, Umklapp scattering processes become more and more 
important. By the increase of phonon momentum and thereby wave vector, threephonon scattering processes can become so strong that the wavevector of the outgoing phonon exceeds the Brillouin zone. By a mathematical addition of a reciprocal lattice vector, the wavevector of the phonon can be transformed back into the Brillouin zone, which is physically equivalent to the result outside of it. In this case of phonon-phonon scattering, the total phonon momentum is not conserved resulting in a thermal resistivity especially at high temperatures [101]. The relaxation times for such Umklapp processes $\mathcal{T}_{u}$ can be expressed by [99, 102]:

$$
\mathcal{T}_{u}^{-1}(\omega)=2 \gamma^{2} \frac{k_{B} T}{\mu V_{0}} \frac{\omega^{2}}{\omega_{D}}
$$

with $\gamma$ being the Grüneisen anharmonicity parameter, $\mu$ the shear modulus and $\omega_{D}$ the Debye frequency of the material. Taking multilayers or superlattices into account, this scattering time can deviate strongly from the bulk value due to socalled mini-Umklapp scattering processes, which happen because of the existence of mini-Brillouin-zones associated with the superlattice structure [95,103].

Considering all those scattering processes and combining them into a single relaxation time $\mathcal{T}_{c}$, one has to apply Matthiessen's rule [102] :

$$
\frac{1}{\mathcal{T}_{c}}=\frac{1}{\mathcal{T}_{b}}+\frac{1}{\mathcal{T}_{i}}+\frac{1}{\mathcal{T}_{u}} .
$$

Overall, phononic thermal conductivity can be efficiently reduced by reducing this combined relaxation time and thus, the mean free path of the phonons. This can be done by increasing the amount of scattering events that can arise when phonons impinge lattice impurities like inclusions, holes, grain boundaries or interfaces as depicted in Figure 2.2 [104-107].

Especially scattering at interfaces represents a powerful tool for manipulating phononic thermal conductivity, since a high amount of interfaces between dissimilar materials can be produced with modern thin film methods $[108,109]$. At room temperature, the typical mean free paths of phonons for metals and semiconductors lie in the range of $10-100 \mathrm{~nm}[110,111]$. This value can be strongly reduced by nanostructuring the material in several thin layers with individual thicknesses below $10 \mathrm{~nm}$. In terms of multilayers those nanostructured systems play a key role in the present thesis as boundary scattering becomes very powerful in materials with high interface densities.

Already in chapter 3 phonon modes are described that are localized within one 


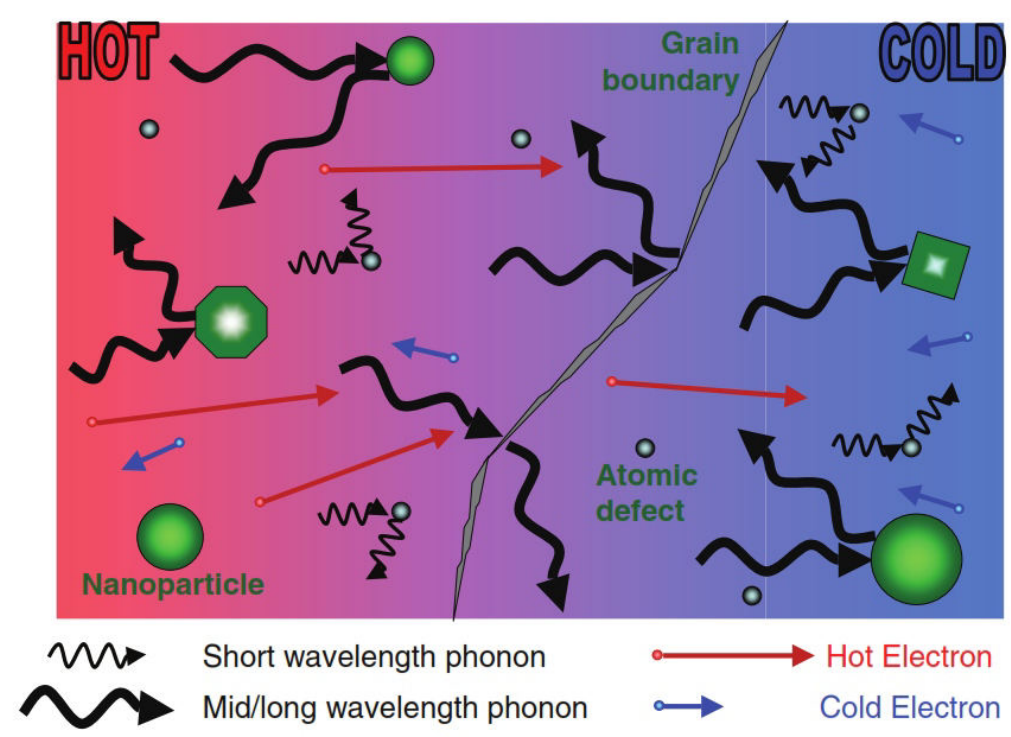

Figure 2.2.: Schematic illustration of heat transport from the hot to the cold side of a material with different scattering centers. Short wavelength phonons can be scattered at atomic defects, but for efficient scattering of longer wavelength phonons, nanoparticles or boundaries are necessary. Preparing material combinations in nanoscale multilayers allows for a high density of scattering interfaces. The multilayers in this thesis always consist partially of isolating materials, suppressing the conduction via hot electrons. (Figure taken from [91])

layer. Here, confinement is given by the size of the system where coherent boundary scattering occurs in terms of reflection and re-reflection. Using the example of a $100 \mathrm{~nm}$ thick polycarbonate (PC) layer, where a specific phonon mode is reflected about 40 times at the boundaries, one can see, how the mean free path of these phonons is shortened by the boundaries. The confinement of phonon modes can be strongly increased in multilayers, especially in aperiodic ones. This will be explained in chapter 4, where a wider bandwidth of phonons is confined due to different layer thicknesses in the aperiodic layer consisting of $\mathrm{W}$ and PC. This happens again due to boundary scattering in terms of reflection because of the high acoustic mismatch between the two materials. The whole concept of boundary scattering will be fully utilized in chapter 6, where multilayers with up to 340 boundaries between $\mathrm{W}$ and $\mathrm{ZrO}_{2}$ are presented. In those, the reduction of thermal conductivity will be significant due to the reduction of mean free path but also because of other effects that will be explained later on. Generally, multilayers present a basis for this thesis and are prominent for their potential to reduce the heat flow through them. Therefore, they shall be explained more in detail in the next chapter. 


\subsection{Multilayers and superlattices}

As stated above, thermal conductivity can be efficiently decreased by reducing the phonon mean free path, which can be done in nanoscale multilayers and superlattices by a high amount of interfaces. The term multilayers is widely and also in this thesis used to denote a compound of stacked thin films of two or more different materials with single layer thicknesses mostly in the range of several $\mathrm{nm}$. In comparison, the term superlattice is broader and describes simply a material or material combination with a strong ordering above the level of the atomic lattice. This can be thin film multilayers, but also macroscopic materials $[17,108]$. The concept of influencing thermal transport by superlattices goes back to the 1970s [112] and has got increased attention since. Nowadays, there are superlattices and multilayers that contain almost all kinds of material classes such as metals [113-115], oxides [15,116], semiconductors [117,118], quantum dots [119] and even liquids [120]. With such a high variety of potential material combinations, superlattices found their way into different applications [121-124]. In the sense of low thermal conductivity materials, the application of superlattices became widely discussed in the field of thermoelectrics [12,125-127]. For such modern energy materials, it is essential to decrease the phononic thermal conductivity $\kappa_{p}$ while maintaining a high electrical conductivity $\sigma$ and a high Seebeck number $S$ [128]. This is expressed in the ZT-value of thermoelectrics, the so-called figure of merit, which is a measure of efficiency of such devices [24,129]. The figure of merit ZT is given by [12]:

$$
\mathrm{ZT}=\frac{S^{2} \sigma}{\kappa_{e}+\kappa_{p}} T
$$

where $\kappa_{e}$ is the electronic thermal conductivity and $\kappa_{p}$ the phononic one.

The inherent property of the figure of merit, namely being proportional to the electrical conductivity while being inversely proportional to the thermal conductivity shows a major challenge in fabricating efficient thermoelectrics. While, according to the Wiedemann-Franz-law [130] the electrical and thermal conductivity are strongly coupled in most metals, multilayer approaches that hinder the phononic propagation have been discussed as being very promising to increase $Z T[109,131,132]$. In superlattices of p-type $\mathrm{Bi}_{2} \mathrm{Te}_{3} / \mathrm{Sb}_{2} \mathrm{Te}_{3}$ even a value of $Z T=2.4$ at $300 \mathrm{~K}$ was achieved, which is a milestone compared to the bulk 
value of $Z T=0.75$ for $\mathrm{Bi}_{2} \mathrm{Te}_{3}$ at $300 \mathrm{~K}[12,133]$. This increase can only be explained by a strongly reduced thermal conductivity through the superlattice.

While this thesis does not investigate thermoelectric materials, however, the effect of minimizing the thermal conductivity is of strong importance here and thermoelectrics are a prominent application in this field. In general, multilayers are a special part of this thesis and are therefore essential for the investigations in all four presented papers (chapter 3-6) as well as for the most recent and yet unpublished results in chapter 7.

Apart from research linked to thermoelectric materials, many different studies have been conducted dealing with heat transport through multilayers. Of those, the most important shall be resumed here, in order to classify the scope of this thesis.

One of the first measurements of thermal conductivity in superlattices has been done on AlAs/GaAs, where a reduction of in-plane thermal conductivity from ca. $30 \mathrm{~W} / \mathrm{mK}$ to a value below $20 \mathrm{~W} / \mathrm{mK}$ was seen, that arose with reducing the layer thickness from $20 \mathrm{~nm}$ down to $5 \mathrm{~nm}$. Both values are lower than the weighted average of the thermal conductivity values of AlAs and GaAs that lies at $67 \mathrm{~W} / \mathrm{mK}$ but still above the value of the $\mathrm{Al}_{0.5} \mathrm{Ga}_{0.5} \mathrm{As}$ alloy at $9 \mathrm{~W} / \mathrm{mK}$ [134]. In the same material system with layer thicknesses ranging from 1 to 40 monolayers crossplane thermal conductivity has been measured. The result of this study showed a reduction by a factor of three in the superlattice with 40 monolayer thick layers and a reduction by one order of magnitude for the sample with 1 monolayer thick layers compared to bulk values of GaAs [135].

Those early studies already showed a reduction in thermal conductivity in the superlattice compared to bulk samples. Moreover, already the trend of reducing the conductivity with increasing layer density is visible.

In order to extend the knowledge in this field and to prove this hypothesis, more systematic studies on the correlation between the thermal conductivity and the interface densities have been conducted. The focus therein lied on interface scattering and thus, reduction of mean free path as well as on the thereof resulting thermal boundary resistances that will be explained later on in this chapter.

In different works an overall decrease of thermal conductivity with increasing interface density could be shown [81]. In addition to this, also steps in thermal conductivity could be seen, like in the Si/Ge system, where samples with superlattice periods between 3 and $30 \mathrm{~nm}$ were measured [136]. Here, the high values of $\mathrm{Si}$ and $\mathrm{Ge}(150 \mathrm{~W} / \mathrm{mK}$ and $60 \mathrm{~W} / \mathrm{mK}$, respectively) were reduced to a value ranging from 1 to $5 \mathrm{~W} / \mathrm{mK}$ depending on superlattice period. As expected, 
there was a reduction of thermal conductivity with increasing interface density when going from $7 \mathrm{~nm}$ period down to $3 \mathrm{~nm}$. However, above $7 \mathrm{~nm}$ there was a drop in thermal conductivity such that the $13 \mathrm{~nm}$ and $30 \mathrm{~nm}$ samples were better insulating than the $3 \mathrm{~nm}$ ones. This, on the first sight contradictory behavior, can be attributed at one side to interface scattering that results in the reduction with a higher interface density. On the other side, it can be attributed to defect scattering, which became dominant in the longer period samples through disorder caused by plastic deformation at the interfaces [137]. This example shall explain how different scattering mechanisms can be dominant resulting in a decrease in thermal conductivity. For this work, this will be interesting in chapter 6, where samples with high interface densities and therefore strong boundary scattering are compared to interfaceless samples, where impurity scattering is predominant.

Besides the general reduction effect of thermal conductivity in multilayers, also the temperature dependence of this effect was of interest and investigated in literature. Interestingly, for some material combinations (consisting of GaAs and AlAs) the thermal conductivity decreased with increasing temperature by a factor of 2 between 200 and $400 \mathrm{~K}$ [135, 138], while in other superlattices (consisting of $\mathrm{Si}$ and $\mathrm{SiGe}$ ) the opposite behavior was the case and a general increase of thermal conductivity with temperature depending on the superlattice spacing was observed [136,139]. Such contradictions indicate a material dependent difference in scattering phenomena that limit heat transport. While in the first case phonon-phonon scattering seems to be the most important mechanism, the different behavior of thermal conductivity in the second experiments suggests defect scattering to be the dominant effect.

This example again shows the different contributions and different dominating scattering mechanisms that have to be considered for reducing the thermal conductivity. For this thesis, an interplay of many different scattering phenomena can be assumed from which probably the boundary scattering is the part that can be best exploited in thin film multilayers due to interface resistances.

Independently of such different scattering mechanisms, a wide variety of work shows how these resistances are important for generally minimizing thermal conductivities in superlattices $[134,138,139]$. Whether or not the final thermal conductivity of the superlattice can be lower than the value of the alloy is strongly dependent on the mismatch of the layer materials, which will be explained later. Thus, it is especially challenging for disordered oxidic materials. Such have mostly already a low thermal conductivity and show no great acoustic mismatch 
compared to other oxides [108, 140].

In contrast to this, the strategy of comparing oxides with metals has led to fruitful results. In this system, a high acoustic mismatch could be realized and thus, strong thermal boundary resistances resulted. Ultra-low thermal conductivity could be achieved in a multilayer consisting of $\mathrm{W}$ and $\mathrm{Al}_{2} \mathrm{O}_{3}$ [39]. In that work it could be shown that individual layers of dissimilar materials, which have thicknesses of only a few nanometers allow reducing the overall thermal conductivity strongly below the amorphous limit. A graph depicting thermal conductivity dependent on interface density is shown in figure 2.3. Likewise results were also found for nanolaminates of $\mathrm{Ta} / \mathrm{TaO}_{x}$ where also a superlattice allowed a strong minimization of thermal conductivity [141].

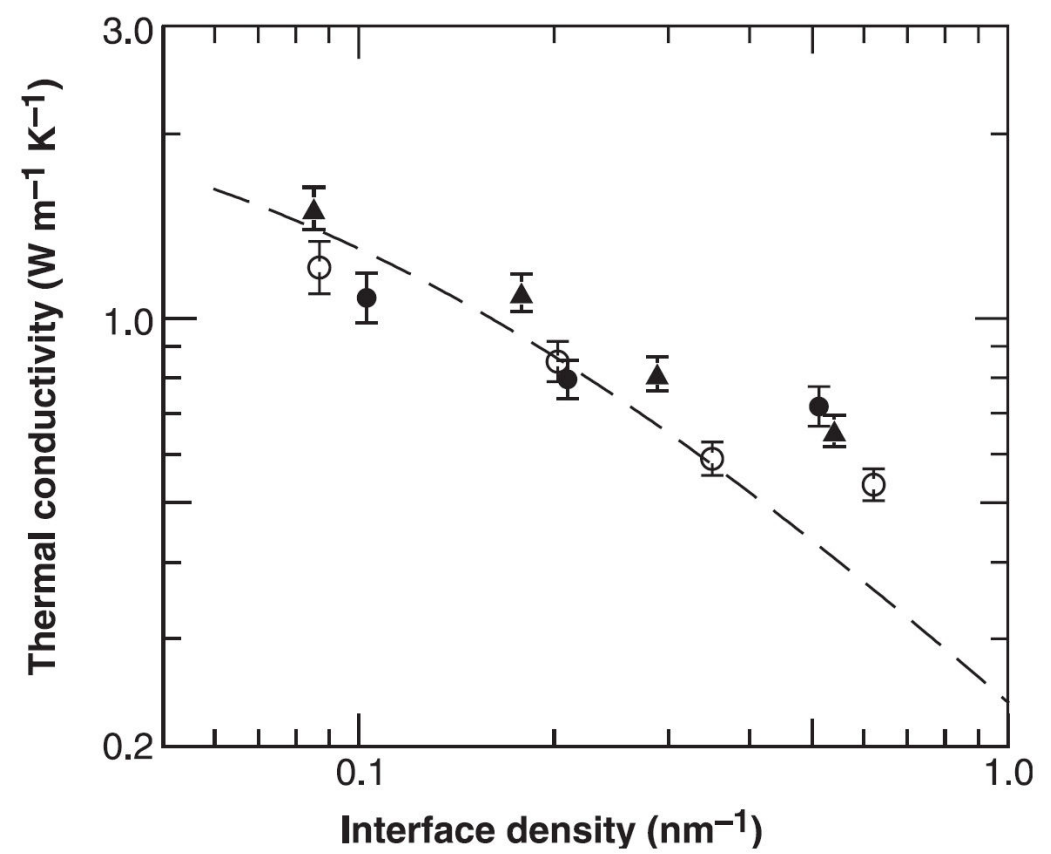

Figure 2.3.: Thermal conductivity is reduced with a higher interface density in a W/ $\mathrm{Al}_{2} \mathrm{O}_{3}$ superlattice at room temperature [39].

Comparing this interesting result of ultra-low thermal conductivity, which was published in the famous Science journal to measurements on $\mathrm{W} / \mathrm{ZrO}_{2}$ multilayers done in this work, explained in chapter 6 , one can see that the thermal conductivity in both cases lie in the same order of magnitude. Also in this thesis, ultra-low thermal conductivity values below $1 \mathrm{~W} / \mathrm{mK}$ in multilayers of metals and oxides could be achieved.

In order to further reduce the thermal conductivity, one can increase interface density even more, by going to the smallest possible layers that exist in monoatomically 
stacked crystals. This could be achieved in the case of the layered $\mathrm{WSe}_{2}$ crystal that was grown from alternating $\mathrm{W}$ and Se layers. Here, one could reduce cross-plane thermal conductivity even further and reached a value of only $0.05 \mathrm{~W} / \mathrm{mK}$, which is the lowest for fully dense materials measured so far [142]. In this system, the thermal conductivity is reduced by a factor of 30 compared to the bulk $\mathrm{WSe}_{2}$ single crystal and even 6 times smaller than the expected minimum for this material, which can be attributed to phonon localization due to the random stacking of the monoatomic sheets.

In this present thesis, an almost comparatively result could be achieved by multilayers of $\mathrm{W}$ and $\mathrm{PC}$ that show likewise a very low thermal conductivities below $0.1 \mathrm{~W} / \mathrm{mK}$ due to the great difference in material properties and the thereof resulting thermal boundary resistance. This result is first estimated in chapter 4 and finally measured in chapter 7 .

The general potential of reducing thermal conductivity in multilayers is shown in figure 2.4, where typical crystalline materials are compared with amorphous materials that have a much lower thermal conductivity. The two most important findings on lowered thermal conductivity in layered systems, namely the ultralow thermal conductivities in $\mathrm{W} / \mathrm{Al}_{2} \mathrm{O}_{3}$ as well as in $\mathrm{WSe}_{2}$ are added in this graph, illustrating the possibilities of such structures.

In order to understand the underlying physics of the reduction effect of thermal conductivity in multilayers and superlattices more in detail, the theory of heat transport through such structures has to be understood. Therefore, the next chapter will explain the models of heat conduction in its different regimes depending on the phonon mean free path and the size of the superlattice spacings. It will explain the most important mechanisms for the observed reduction effect.

\subsection{Heat transport models}

In order to understand thermal transport in mutlilayers and superlattices and thereby the desired reduction in thermal conductivity, one has to differentiate between two transport regimes, namely the incoherent, or diffusive regime and the coherent, or ballistic regime [5]. The first one becomes physically more relevant when the mean free path of the phonons is shorter than the characteristic length scale of the superlattice: $l<d$. Here, phonons can be treated as particles that scatter several times inside the layers as well as at the interfaces. This will become very 


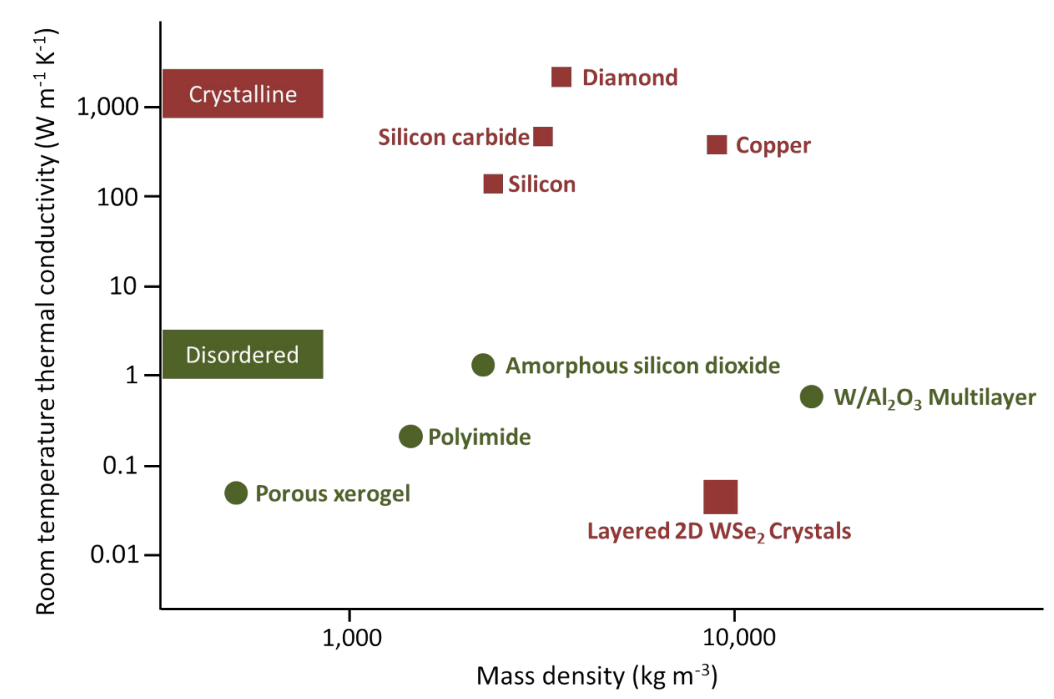

Figure 2.4.: Thermal conductivity values of some representative bulk materials at room temperature. Compared to those, the values of $\mathrm{W} / \mathrm{Al}_{2} \mathrm{O}_{3}$ multilayers with high interface density is added showing that a combination of a high conducting material (W) with an insulating oxidic material $\left(\mathrm{Al}_{2} \mathrm{O}_{3}\right)$ can have a lower thermal conductivity than the oxidic material or the amorphous limit of those materials [39]. The case for the greatest interface density, namely in monoatomically layered $\mathrm{WSe}_{2}$ crystals is also shown, because it presents the lowest thermal conductivity of fully dense materials known so far [142]. (Figure adapted from [35])

important in chapter 6 of this thesis, since there the diffuse transport is dominant and thereby the reduction of thermal conductivity can be best explained with this approach.

When the mean free path approaches the length scale of the superlattice $(l \sim d)$, thermal transport becomes quasi-ballistic such that wave- and particle properties of phonons have to be considered. In this regime, transport inside the layers is mostly ballistic, becoming diffuse at the interface due to boundary scattering. For even smaller structures in the superlattice or larger mean free paths $(l>d)$, heat transport becomes more and more ballistic and phonons have to be treated as waves. Such wavelike behavior is investigated in chapter 3 and 4 of this thesis, where localized standing phonon waves indicate strong confinement of phonon modes.

However, note that the mean free path of phonons does not only depend on the structure of the material, but also on temperature and phonon frequency, more than one of those regimes can contribute to thermal transport at the same time. 


\subsubsection{Diffusive Transport}

Taking into account the diffusive regime for describing thermal conductivity through a multilayer, phonons undergo various scattering regimes before impinging an interface. Thus, local temperature can be defined within individual layers and a temperature gradient for thick enough layers exists [81]. Therefore, the thermal conductivity of the multilayer can be expressed by bulk components from the layers and interface components. Both components pose a resistance against heat flow, as shown in figure 2.5. The high temperature on one side of the material is reduced in the bulk via a temperature gradient that arises from the bulk thermal resistance as well as a temperature drop at the interface [143]. In multilayers,

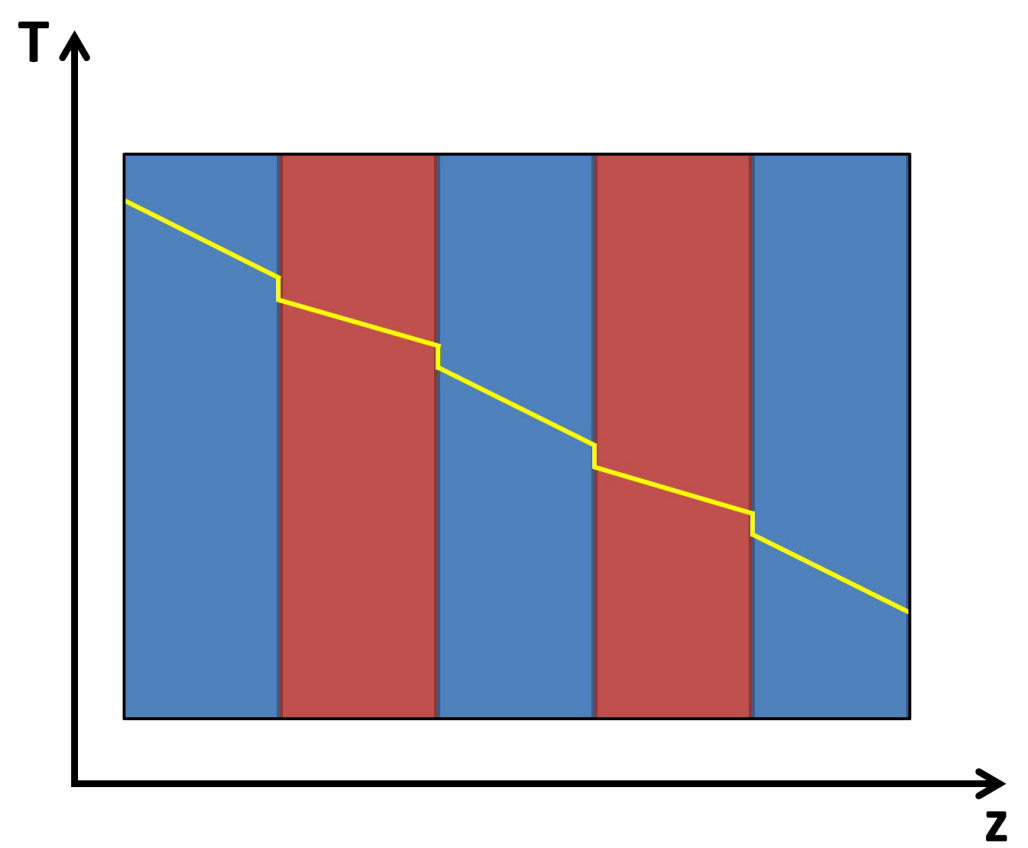

Figure 2.5.: In the case, where the superlattice spacing is greater than the phonon mean free path, thermal transport can be described by the diffuse scattering model. Due to bulk scattering in each layer the temperature drops linearly with $\mathrm{z}$ within the layer. Additional thermal resistances at each interface result in temperature drops between two materials. The effective thermal conductivity can be described for a given heat flux density by the overall temperature decrease over the size of the superlattice.

especially the second part is interesting, since with low layer thicknesses and a high interface density, the interface effects become more and more important. For the case of diffusive transport, particularly scattering at the interfaces is important. This will be very important for chapter 6 and 7 of this work, where $\mathrm{W} / \mathrm{ZrO}_{2}$ multilayers of overall thickness of $850 \mathrm{~nm}$ with up to 340 interfaces and W/PC 
multilayers of $1.2 \mu \mathrm{m}$ with 800 interfaces will be shown.

Apart from the number of interfaces also interface roughness and intermixing play an important role. By intermixing, a very small alloy layer is brought into the system at every interface. Roughness is very dependent on both, the growth method and the utilized materials and therefore differs greatly in various multilayers. Moreover, asymmetric interfaces can make matters even more complicated, because now there are two different interfaces, depending on which material grows on which and therefore, also two different interface resistances have to be considered $[81,144]$. Another effect that enhances interface scattering and therefore reduces the thermal conductivity is strain at the interfaces, which can arise for instance from different lattice parameters of the two components [136].

Applying this knowledge to the work done here, one can say that PLD is a suitable method for the production of well insulating multilayers. Due to the high particle energies of ions during PLD, interface intermixing can be obtained, adding a very small alloy layer between two materials, resulting in a reduced thermal conductivity in a multilayer [145]. At the same time, interface roughnesses can be kept relatively low (at typical values of $0.3-0.8 \mathrm{~nm}$ ) enabling fabrication of very small layers and thus, high interface densities. This will be exploited in chapter 6 and 7 , where ultrathin blocking layers of $0.6 \mathrm{~nm} \mathrm{~W}$ are prepared and used to resist phonon transport. The effect of this blocking layers can qualitatively be seen in figure 2.6, where a higher interface density between laser deposited $\mathrm{W}$ and $\mathrm{ZrO}_{2}$ layers ensures that the surface temperature stays elevated for a longer time, which is a measure for a reduced thermal conductivity.

Another effect that results in a reduction in thermal conductivity is strain within layers. Again due to the energetic particles that occur with PLD, layers on both sides of the interface can be strained. Due to implantation of ions (the so called 'shot peening'), compressive stress can occur and due to coalescence during thin film growth (and the so called 'island zipping' mechanism) tensile stress can appear [53,146,147]. Such stress and the resulting strain on both sides of the interface reduces the propagation probability by enhancing the thermal boundary resistance of the interface.

In order to predict the thermal resistance of the whole layered material, one can 


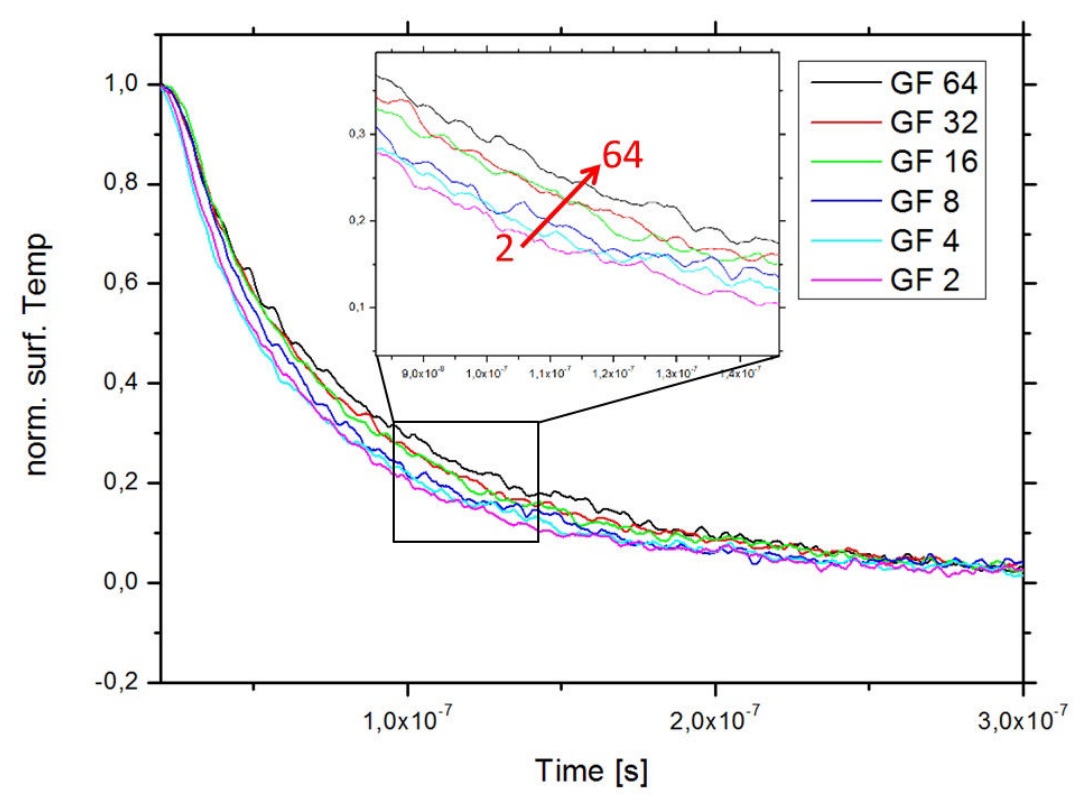

Figure 2.6.: Qualitative analysis of 6 different pulsed laser deposited $\mathrm{W} / \mathrm{ZrO}_{2}$ samples with same overall thickness of $280 \mathrm{~nm}$ but a varying number of interface ranging from 2 to 64 . The TTR method (see chapter 5) was used in order to measure the time-dependent surface temperature. A trend, enlarged in the inset of this figure, indicates that with higher interface density the surface temperature is less efficiently transportet away from the surface which a reduction of thermal conductivity with higher interface density.

model it as a resistor chain, where one repeat period of the superlattice has the value $R_{P}$ that can be expressed in the following way [148]:

$$
R_{P}=\frac{d_{1}}{\kappa_{1}}+\frac{d_{2}}{\kappa_{2}}+2 R_{B}
$$

Here, $d_{1}$ and $d_{2}$ denote the individual layer thickness of the two components of which the superlattice consists, together with their corresponding thermal conductivities $\kappa_{1}$ and $\kappa_{2}$ and the boundary resistance $R_{B}$ that will be discussed in the next chapter. Applying this formula to a superlattice with $\mathrm{N}$ periods of periodicity $\Lambda=d_{1}+d_{2}$ and a total thickness of $D=N \Lambda$, one can obtain the thermal conductivity of the whole superlattice is obtained by:

$$
\kappa_{S L}=\frac{1}{\frac{x}{\kappa_{1}}+\frac{1-x}{\kappa_{2}}+n R_{B}} .
$$

Now, the relative thickness of component $1 x=N d_{1} / D=d_{1} / \Lambda$ and $1-x$ for component 2 as well as the interface density $n=2 N / D=2 / \Lambda$ have been introduced. This formula is important for a large part of this thesis, since it 
describes diffusive thermal conductivity in multilayers. Because of the size of the layers presented here and the roughness between them, thermal transport can be assumed to be mostly diffusive, making this formula elemental in the following chapters. Already in chapter 4 it is used in order to estimate the thermal conductivity of W/PC multilayers. The result of this estimation supports an evaluation based upon the cumulative heating in those samples. Both approaches to assess the cross-plane thermal conductivity of the W/PC multilayers of this thesis yield an ultra-low value below $0.1 \mathrm{~W} / \mathrm{mK}$, which lies close to the so far lowest value of fully dense materials of the above described monoatomically layered $\mathrm{WSe}_{2}$ crystal [142]. This new and remarkable result can be explained by the great mismatch of the hard and heavy material $\mathrm{W}$ in contrast to the soft and lightweight polymer PC and the thereof resulting high thermal boundary resistance, which is measured with the TTR method in chapter 7. Moreover, equation 2.12 is used in chapter 5 for the quasi-material approach that allows to measure thermal conductivities of multilayers. Furthermore, this approach is applied to the $\mathrm{W} / \mathrm{ZrO}_{2}$ multilayers in chapter 6 where the evaluation of the thermal conductivity values is based upon this formula as well as to layered samples in the addendum (chapter 7).

In order to utilize equation 2.12, one needs precise information about individual layer thicknesses of the multilayer. This is done in this thesis by highly-precise X-ray reflectivity measurements as well as measurements with high-resolution transmission electron microscopes.

A potential drawback of this formula is that it takes only interface thermal resistances into account, but does not account for defects, impurities and other scattering centers inside the layers. Due to those deviations from the perfect lattice, the mean free path of the phonons in the constituent materials is shortened and thus, the thermal conductivities of the single layers deviate from their bulk values [149]. In order to account for this effect, one might utilize adjusted values for $\kappa_{1}$ and $\kappa_{2}$. Another drawback is that it does not account for coherent effects, which shall be described later. In the case of this thesis, this is not crucial, because diffuse transport is dominating here. 


\subsubsection{Boundary Resistance}

The value $R_{B}$ in equation 2.11 and 2.12 is the boundary resistance of the interface, which is the macroscopic expression of the hindered phonon transport across the boundary. The heat flow perpendicular through a planar interface with a temperature difference $\Delta T$ between each side of the interfaces can be expressed as [150]:

$$
\dot{Q}=\frac{1}{R_{B}} \Delta T
$$

where $R_{B}$ denotes the boundary resistance that leads to the temperature difference and is called Kapitza resistance [151]. The calculations of such boundary resistances from material properties started with the acoustic mismatch theory [152] and were continuously improved in order to include more phonon modes on each side of the interface [153], different lattices [154] and phonon-phonon interactions for rough surfaces [155]. The basic idea behind those calculations is that the materials next to the interface have dissimilar properties in terms of sound velocity $v$ and density $\rho$ resulting in a mismatch between the acoustic impedances $Z=v \rho$ on each side. The stronger this mismatch is, the lower becomes the phonon transmission probability across the interface [86]. This is described in the acoustic mismatch theory with a transmission probability coefficient $t_{A \rightarrow B}$ for phonon energy impinging perpendicular on the interface from material A to material B [156]:

$$
t_{A \rightarrow B}=\frac{4 Z_{A} Z_{B}}{\left(Z_{A}+Z_{B}\right)^{2}}
$$

This theory, which emerged in analogy to optics, assumes no scattering at interfaces and imposes therefore perfectly smooth interfaces with appropriate stress and strain boundary conditions [81]. In contrast to this, another model, the diffuse mismatch model describes the interface thermal resistance in the approximation that all phonons scatter at the interface [86]. The probability to be scattered to material A or B is then proportional to the phonon density of states at each side. Thus, by fabricating multilayers whose consisting materials have a huge mismatch, like for instance combinations of hard and soft materials or heavy and light ones, the boundary resistance can be significantly increased and therefore the thermal conductivity reduced. With a significantly strong mismatch in the phonon dispersion relation on both sides of the interfaces, phonons in broad frequency 
ranges are confined and cannot propagate across the interface. With a high interface density in superlattices, it should therefore be possible to reduce the cross-plane thermal conductivity strongly below the bulk values of the constituting materials $[117,157,158]$.

In this thesis, the reduction of phonon transmission across interfaces is very important and one of the central goals for minimizing thermal conductivity. In chapter 3 phonon reflection is studied in the metal polymer system $\mathrm{Cu} / \mathrm{PC}$ which excels by a strong acoustic mismatch. This property difference can still be increased by taking a heavier metal as counterpart to PC, which was done in chapter 4. Here, W/PC multilayers were investigated that have because of their large difference in density and sound velocity a theoretical transmission coefficient of only $6 \%$ according to equation 2.14. This explains the formidable phonon mode confinement and the low thermal conductivity that could be achieved within those multilayers. In chapter 6 the material combination $\mathrm{W} / \mathrm{ZrO}_{2}$ is used, which does not have such a high acoustic mismatch as the W/PC combination and thus also not such a high boundary resistance. However, in terms of high temperature applications they are much better suited as they exhibit better properties on behalf ot their thermal stability. In this system, the thermal boundary resistance could be measured by the TTR method, which is explained in chapter 5 . With a value of ca. $1.6 \cdot 10^{-9} \mathrm{~m}^{2} \mathrm{~K} / \mathrm{W}$ it is in the same order of magnitude as interfaces in other multilayer systems $[39,159,160]$.

In order to fully understand the effect of minimized thermal conductivity through superlattices, the concept of diffusive scattering and boundary resistance alone is not sufficient and not capable to completely describe the reduction in thermal conductivity below the amorphous limit. That is why, also the non-diffusive regime has to be taken into account, which is described below.

\subsubsection{Ballistic Transport}

Depending on the superlattice spacing, temperature and occupied phonon frequencies, the mean free path can approach the length scales of the superlattice $(l \sim d)$. Now, transport is not fully diffuse anymore, because scattering inside the layers becomes less probable due to the large mean free path. This regime is commonly referred to as the quasi-ballistic regime [5]. Here, phonons cross the layers as coherent waves without undergoing any scattering events. In this case, local 
thermal equilibrium is not existing any longer and local temperature fields cannot be defined on the nanoscale [81]. Therefore, also the linear temperature decrease inside a layer, known from the diffusive regime, is not given anymore. Now, scattering events can mainly occur when phonons impinge an interface. By this, the interfaces can reduce the mean free path of the phonons, when the scattering is incoherent, meaning that the phase information gets lost. In this picture, the mean free path becomes the superlattice spacing, which allows to effectively reduce it with always thinner layers and thus, reduces the thermal conductivity.

For even smaller superlattice spacings and larger phonon mean free paths $(l>d)$, phonon waves propagate coherently across several layers and interfaces. This regime is called the ballistic regime, where mainly coherent phonon transport has to be considered and phonons are treated as waves. When such waves impinge an interface, they can transmit or be reflected as described by the above mentioned models of acoustic and diffuse mismatch. Such behavior is shown in figure 2.7, where phonons, treated as waves, impinging an interface can be transmitted or reflected.

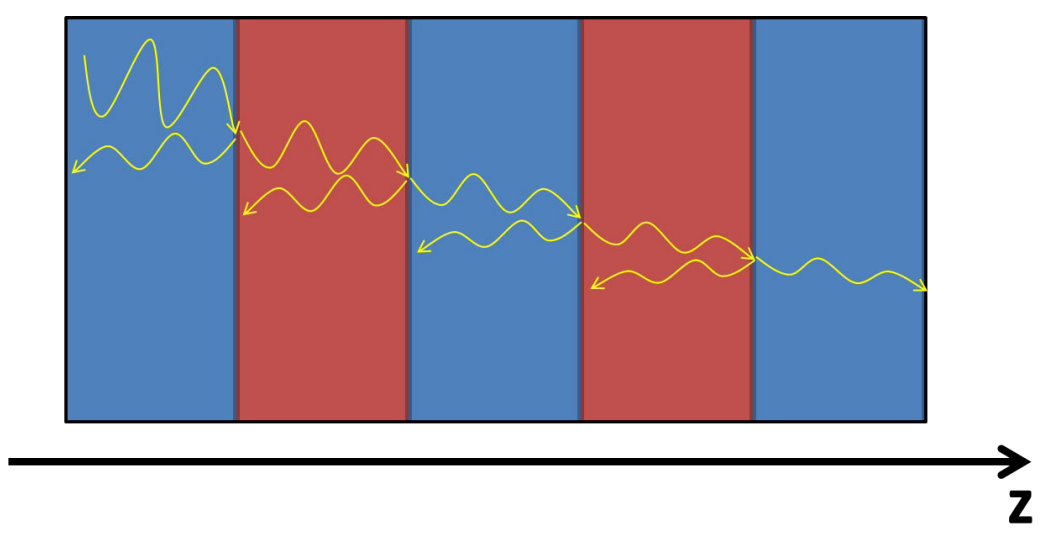

Figure 2.7.: Coherent heat transport becomes dominant when the phonon mean free path is longer than the superlattice spacing. Here, phonons must be treated as waves and can be transmitted or reflected at each interface. By multiple reflections, interference occurs leading to a modification in the dispersion relation and thereby to a reduction in heat transport via effects like phonon localization or a reduction in group velocity.

In this thesis the concept of reflecting phonon waves is studied mainly in chapter 3 and 4, where confinement and localization of such waves are demonstrated. But also for the results of chapter 6 the consideration of such effects is of importance in order to understand the reduction of thermal conductivity.

When, in the ballistic regime, the mean free path of phonons is long enough to span several superlattice spacings, re-reflection can occur and even interference 
effects due to the periodic structure of the interfaces becomes possible. Those effects change the dispersion relation of the material leading to artificial phonon band gaps depending on the superlattice structure as well as on the constituting materials and their properties [161]. Thus, by the ability to structure superlattices artificially from a variety of materials, one can influence how different phonon modes contribute to heat transport through the superlattice.

The band gaps that open up due to the superlattice spacing can strongly differ from the classical dispersion relation of the material components. As the typical lattice spacing is on the order of Ångströms and the typical superlattice spacing lies in the range of several nanometers, the Brillouin zone, whose size scales inversely with the size of the structures in the position-space, becomes strongly minimized in comparison to the Brillouin zone of the bulk material $[42,162]$. This behavior leads to zone folding also resulting in a modification of the dispersion relation and the existence of band gaps [148].

Apart from band gaps that deny phonon propagation another effect reduces the thermal conductivity, namely the reduction of the group velocities of phonons near the edge of the Brillouin zone. The group velocitiy $v_{g}$ of phonons can be expressed as a derivation of the angular frequency $\omega$ with respect to the wavevector K [102]:

$$
v_{g}=\frac{\mathrm{d} \omega}{\mathrm{d} k} .
$$

Since the slope of the angular frequency becomes zero at the boundaries of the Brillouin zone, the group velocities of phonons with a high $k$-value become lower. Thus, those phonons can less effectively carry heat and the overall thermal conductivity decreases.

Another consequence of the altered dispersion and the smaller Brillouin zones is the increase of scattering rates, especially for Umklapp scattering [95]. This effect can further reduce thermal conductivity in superlattices.

All of those effects contribute to the effect of reduced thermal conductivity in superlattices and help understanding the minimized thermal conductivity of the here presented multilayers explained in chapter 4 and 6.

Interestingly and in contrast to the beforehand explained physics, thermal conductivity can also increase with increasing interface density, which is shown in figure 2.8. Here, a minimum in thermal conductivity occurs at the crossover from incoherent to coherent phonon transport [163]. This can be the case for very low layer thicknesses with almost no roughness, so that coherent wave effects 


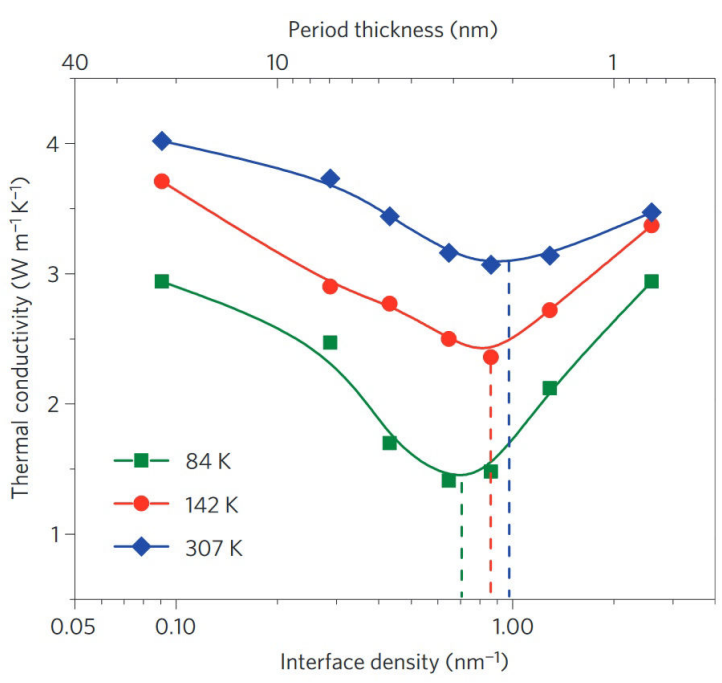

Figure 2.8.: With higher interface densities, a crossover from particle-like diffuse scattering towards wave-like coherent scattering occurs. This was shown in epitaxial oxide superlattices by a minimum in cross-plane lattice thermal conductivity. The minimum arises from a reduction in diffusive transport with interface density up to a certain value when coherent scattering becomes dominant. From this point, an increase of interface density results in a decreasing number of mini-bands and thus to an increase in phononic group velocity [163].

become more important than the diffusely scattered particle-behavior of phonons. If the superlattice period gets small enough that it becomes comparable to the phononic coherence length, a minimum in thermal conductivity can occur. This is caused by the fact that the value of thermal conductivity is at first lowered with higher interface density, as long as the diffusive transport plays the important role. However, at a certain point when band folding occurs and coherent effects become more important, a reduction of layer thickness results in larger Brillouin zones, less mini-bands and an increase in phonon group velocity, which leads to an increase of thermal conductivity. Thus, in the crossover between those two regimes, a minimum must be present.

In order to hinder even this coherent phonon transport, superlattice structures that are not fully periodical can be used. Here, detuning of individual layer thicknesses, layers with random thicknesses or aperiodic stackings allow to suppress coherent phonon propagation even more and should lead to a further decrease in thermal conductivity $[164,165]$. This principle is shown in figure 2.9. One can see, that coherent modes that would normally contribute to heat transport are now localized in the different lattice spacings of the multilayer and cannot propagate due to the aperiodic layering of the superlattice. In the case where coherent transport is dominant, such propagation confinement should lead to a strongly reduced 
thermal conductivity as was calculated in literature by nonequilibrium molecular dynamics $[164,165]$.

a) Periodic multilayer:

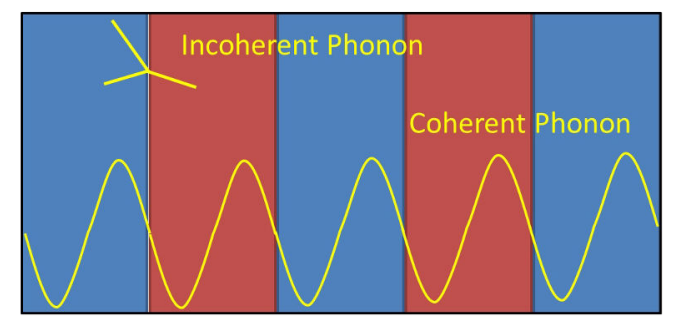

Aperiodic multilayer:

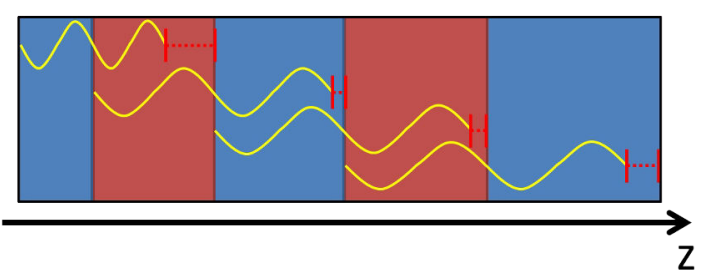

b)

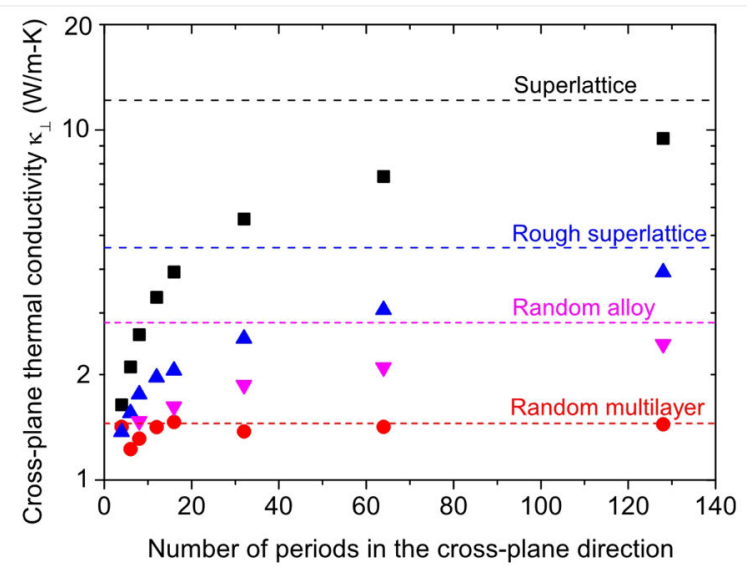

Figure 2.9.: Periodic and aperiodic multilayers show significant differences in terms of coherent phonon tranport. While periodic multilayers allow certain frequencies to propagate almost unhindered, aperiodic multilayers provide strong resistance to coherent phonon transport. The ununiform layer thicknesses result in a misfit of phonon wavelength and superlattice spacing as illustrated with red markings in a) (Adapted from [164]). This results in a strong reduction of cross-plane thermal conductivity as calculated with nonequilibrium molecular dynamics methods and is shown in b). (Taken from [165])

This thesis provides experimental verification of the theories of reduced phonon propagation and gives therefore a good explanation for those theoretical calculations as explained in chapter 4. Regarding the approximately $900 \mathrm{~nm}$ thick multilayer stacks in chapter 6 one can expect that also here deviations from the ideally perfect periodicity are present, which occurred during the deposition process of up to 340 individual layers. This of course affects and disturbs coherent phonon transport resulting on the one hand in an overall reduction of thermal conductivity and on the other hand in a suppression of coherent transport making the considered diffusive transport mechanism more important.

All in all, the idea of aperiodic multilayers presented in this thesis could be a way to further decrease thermal conductivity, but also to control heat flow for thermal management, where new nanostructures are desired for specialized novel applications $[40,166]$. 


\subsection{Transient thermoreflectometry}

Measuring thermal conductivity especially in nanoscale layered structures is a non-trivial task and requires both, a capable measurement method and precise information about layer thicknesses and composition of the sample. In this work, those structural information could be gained using transmission electron microscopy as well as X-ray reflectivity. However, at the beginning of this thesis there was no measurement method for thermal conductivities in the working group of the author. Consequentially, one goal of this thesis was to implement such a method.

In literature, there are several measurement methods described, like among others the 3-omega technique, time-domain thermoreflectance (TDTR) methods, pyrometry, laser flash methods, photodisplacement or transient thermoreflectance methods [63,64,66,73,167-169]. Around those different measurement methods a wide research field evolved lighting their individual advantages and disadvantages. For this thesis, a method for measuring thermal conductivities was desired that is highly flexible and capable of measuring very different material combinations. The reason for that is the great extent of different material classes that could be produced with the versatile PLD method. As shown in chapter 4 and 6 multilayers of metals, oxides and polymers could be prepared and should be investigated using this newly implemented method. In order to fulfill this criterion, the transient thermoreflectance (TTR) method was chosen, which is a very flexible method, able to measure nondestructively and with a high throughput capability thermal conductivity of different material classes including thin film multilayers [71,170-172]. Comparing the TTR-method shown here with the two most common thin-film thermal conductivity measuring techniques, namely the 3-omega method and TDTR, it becomes apparent that each method has its own benefits and drawbacks $[64,169]$. Compared to the 3-omega technique, TTR is much more flexible and can be applied to a much greater family of samples, because no lithographic etching process is necessary and the sample surface underlies no restrictions in terms of its electrical conductivity. especially for the here presented W/PC multilayers this is of particular interest as the process of etching excludes polymers and the electrically conducting W could not be used as top layer either. Without a metallic top layer however, the necessary optical pump-probe measurements that investigate phonon transport would not be possible, because here, the metal layer is needed as a transducer and in order 
to absorb the pump and reflect the probe beam. Therefore, TTR, which is also an optical pump-probe method, is a better suited measurement method for this work. However, TTR features more incertitudes that make it less precise than 3-omega. Compared with TDTR, our TTR is less complex, cheaper to implement and allows in its theoretical evaluation to neglect ultrashort processes like electron-phonon interactions or phonon dynamics and enables measuring diffusive processes on longer timescales. In contrast to these advantages, the samples for our TTR method have to be comparatively thick because of the longer laser pulse duration and ps processes cannot be accessed. However, both of those methodological drawbacks represent no great disadvantages for this work, since the necessary thickness can be achieved with PLD although it is laborious. The investigation of ultrafast processes was realized with a complementary method in collaboration with Henning Ulrichs and Markus Münzenberg as described in chapter 3 and 4. Despite the fact that those drawbacks could be circumvented in this work, again, the precision of TTR measurements is lower than that of TDTR ones, because the evaluation of the measured data is done differently, which excludes some error sources in TDTR that exist for TTR.

All in all, the TTR method that will be shown in chapter 5, is capable of measuring thermal conductivities of very different materials and multilayers with sufficiently high accuracy. The basics and the scientific background of this method shall be explained here more in detail.

The first implementation of TTR goes back to 1986 where thin metal films were investigated in terms of their thermal diffusivity [67]. The principle of this method has not changed much since then. A short laser pulse is used in order to heat up the surface of the sample. Via a second laser, the time dependent change in reflectivity is measured at the same location. This principle is sketched schematically in figure 2.10 .

From this time-dependent reflectivity change, a change in temperature at the surface can be calculated, which is used in order to obtain information about the thermal conductivity of the whole sample. In order to understand that method, information about laser heating, the temperature dependent reflectivity change and the route from the surface temperature to the thermal conductivity shall be given here.

When a laser pulse hits an opaque surface, a part of its energy is reflected 


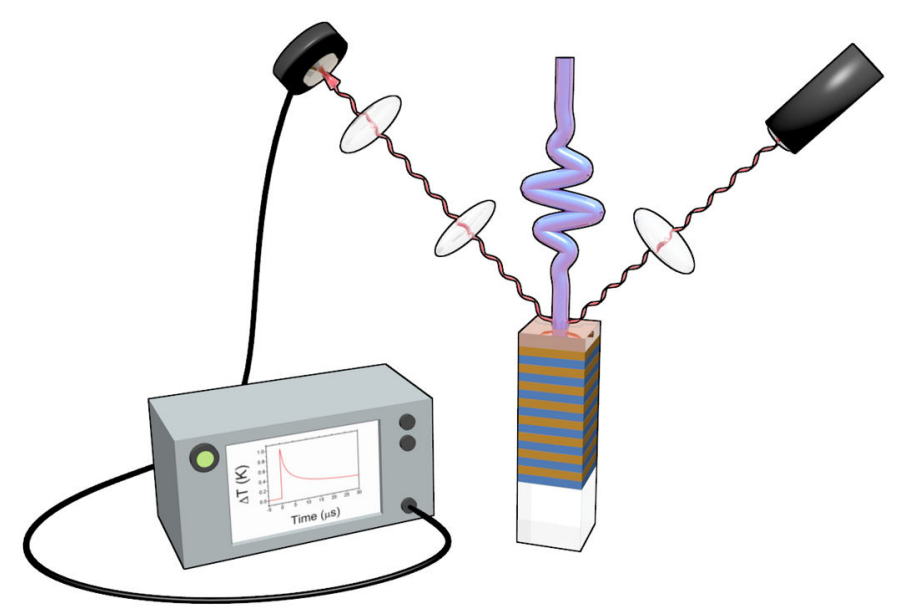

Figure 2.10.: Sketch of the TTR setup. A high fluence laser pulse with $248 \mathrm{~nm}$ wavelength and 20 ns pulse duration heats up the surface, which changes its reflectivity. This change is traced time-dependently via a continuous wave laser of $643 \mathrm{~nm}$ wavelength and analyzed with a fast oscilloscope [173].

but another part is absorbed [174,175]. This absorption leads to an elevated temperature $T(x, 0)$ at position $x$ of

$$
T(x, 0)=\frac{J(1-R)}{c \delta} e^{-x / \delta}
$$

with a laser flux $J$ impinging the sample surface, the reflectance $R$ of the surface, the light penetration depth $\delta=\lambda / 4 \pi k$ according to the Lambert-Beer law with the laser wavelength $\lambda$ and the imaginary part of the index of refraction $k[176,177]$. The reflectivity $R$ of the surface, which depends on the real and imaginary part of the refractive index $n$ and $k$ is calculated according to Fresnel's formulas. Simplified for normal incidence of the laser it has the following form [178]:

$$
R=\frac{(n-1)^{2}+k^{2}}{(n+1)^{2}+k^{2}} .
$$

Since the refractive index and thus, $n$ and $k$ are temperature dependent, also a temperature dependence of $R$ is the case [179]. This can be expressed as a Taylor series expansion in the following way [180]:

$$
\frac{R(T)-R\left(T_{0}\right)}{R\left(T_{0}\right)}=C_{1}\left(T-T_{0}\right)+\frac{C_{2}}{2}\left(T-T_{0}\right)^{2}+\ldots
$$


The factors $C_{1}$ and $C_{2}$ can be expressed by $C_{1}=R\left(T_{0}\right)^{-1} \partial R /\left.\partial T\right|_{T_{0}}$ and $C_{2}=$ $R\left(T_{0}\right)^{-1} \partial^{2} R /\left.\partial T^{2}\right|_{T_{0}}$. Since $C_{2}<<C_{1}$, usually a first order approximation of equation 2.18 is sufficiently accurate. In this way, the equation can be rewritten in the form, which is utilized in this thesis in chapter 5 and 6 :

$$
\frac{\Delta R}{R}=\frac{1}{R} \frac{\partial R}{\partial T} \Delta T=C \Delta T,
$$

where $\Delta R=R(T)-R\left(T_{0}\right), C=C_{1}$ and $\Delta T=T-T_{0}$.

This formula is a key element of this thesis, since it connects the measurable change in reflectivity $\Delta R$ with the desired thermal information about the change in temperature $\Delta T$ via the so-called thermoreflectance coefficient $C$. Unfortunately, this coefficient is mostly very low and lies for typical metals and wavelengths between $10^{-4}$ and $10^{-5}$, making it hard to detect thermoreflectivity [181]. In order to obtain a sufficiently high change in reflectivity $\Delta R$, a metal transducer on top of the sample is essential, which has the highest possible thermoreflectance coefficient $C$ as well as a high reflectivity $R$ for the probe wavelength. Moreover, it should be thermally stable, since an elevated temperature $T$ also increases the signal obtained in TTR. Note, that the thermal conductivity can also be highly temperature dependent for some materials as explained via different scattering mechanism earlier in this chapter and not all samples can be exposed to high temperatures.

Since the TTR method presented in this thesis utilizes a new combination of laser wavelengths and pulse lengths it was also a new challenge to implement it and to obtain a usable signal. These challenges and how they were coped with are described in chapter 5 .

From the measured change in reflectivity, one can conclude on the time-dependent temperature evolution at the surface. In order to infer the thermal conductivity from this information one has to solve the heat equation, which can be derived from Fourier's law that has already been expressed earlier in equation 2.1. The heat equation describes the time- and space-dependent temperature field in a solid and reads [74]:

$$
\frac{\partial T}{\partial t}-\alpha \nabla^{2} T=0 .
$$

The variable $\alpha$ is called thermal diffusivity and consists of the thermal conductivity $\kappa$, the heat capacity $c$ and the density $\rho$ in the form $\alpha=\kappa /(c \rho)$. 
For all here demonstrated TTR experiments the heat transport can always be considered as one-dimensional, since the pump pulse that heats up the surface is much broader than the probe pulse, which detects the reflectivity change at the surface. Therefore, the term $\nabla T$ simplifies to $\partial^{2} T / \partial x^{2}$ but equation 2.20 still stays a parabolic partial differential equation. In order to solve it and get the solution $T(x, t)$, one can Fourier transform it with respect to $x$ considering the derivative theorem resulting in an ordinary differential equation, which is solvable. After a reverse Fourier transformation that takes into account the convolution theorem, the final result reads:

$$
T(x, t)=\frac{1}{2 \sqrt{\alpha \pi t}} \int_{-\infty}^{\infty} e^{-\frac{(x-y)^{2}}{4 \alpha t}} f(y) \mathrm{d} y
$$

Here, in this typical solution of the heat equation $f(x)=T(x, 0)$ is the initial temperature field at $t=0$. Approximating the heating laser as an instantaneous Dirac-pulse of strength $\bar{Q}=Q / \rho c$ at $t=0$, equation 2.21 becomes the following form [74, 182]:

$$
T(x, t)=\frac{Q}{\rho c \sqrt{\alpha \pi t}} e^{-\frac{(x)^{2}}{4 \alpha t}}
$$

In this formula the parameters $\rho, c$ and $\alpha=\kappa /(\rho c)$ can be combined in the value of the thermal effusivity $e=\sqrt{\kappa \rho c}$. Improving equation 2.22 in order to account for the $\tau=20$ ns pulse time of the laser utilized in this work, which is described as a square wave pulse $\theta(t)$, one can apply Duhamel's theorem as explained in chapter 5, which yields $[183,184]$ :

$$
T_{D}(z=0, t)=\int_{0}^{t} \theta\left(t^{\prime}\right) \cdot T\left(z=0, t-t^{\prime}\right) d t^{\prime}=\frac{2 Q}{\tau e \sqrt{\pi}}(\sqrt{t}-\sqrt{t-\tau}) .
$$

This final formula has been derived using several approximations that can lead to errors in the final evaluation and therefore have to be discussed. The two most important ones are first, the approximation of infinite thickness of the sample and second, the neglecting of heat losses.

The finite thickness effect has already been discussed in literature with the result that this approximation may be ignored for sample thicknesses $L$ obeying $L>$ $\sqrt{10 \alpha \tau}$, which is greater than the thermal penetration depth $[183,185]$. This has been studied in this thesis during the setup of the TTR method with different thicknesses of $\mathrm{ZrO}_{2}$ on a Si substrate as shown in figure 2.11. 

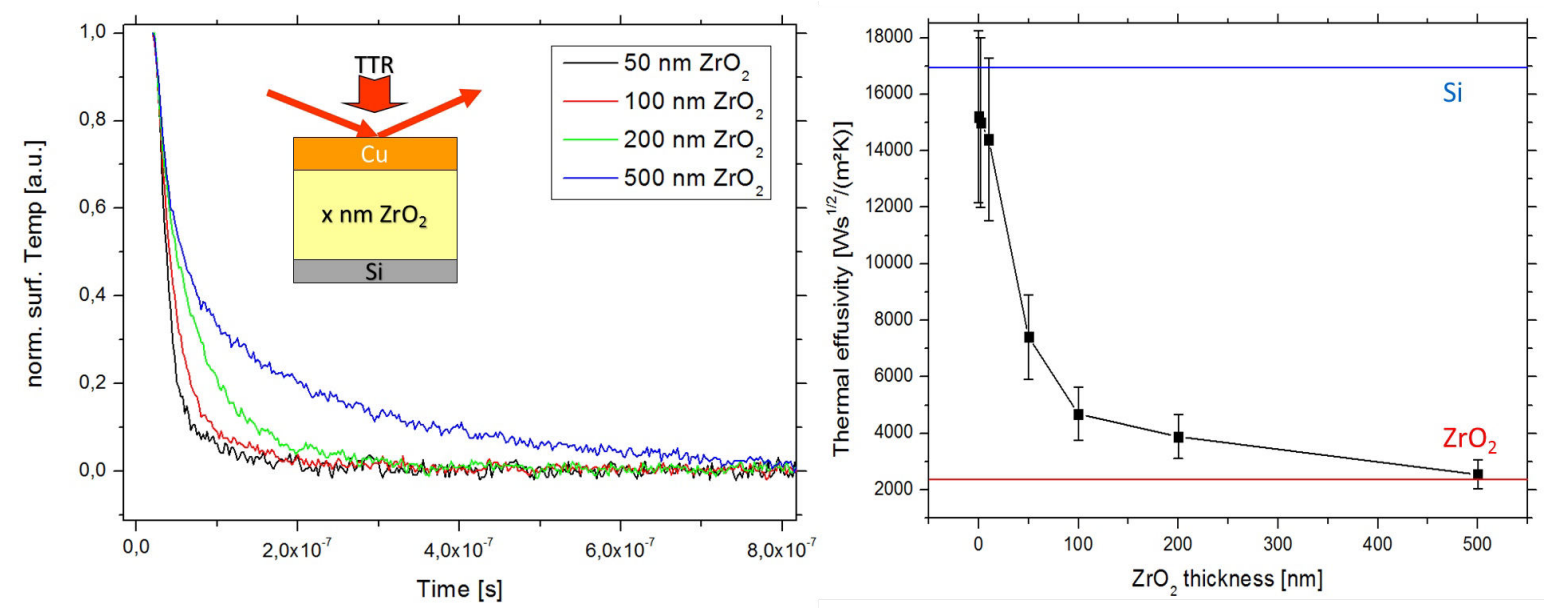

Figure 2.11.: Different thicknesses of $\mathrm{ZrO}_{2}$ on $\mathrm{Si}$ have been measured with TTR showing that a minimum thickness is necessary to neglect the influence of the substrate. Several chosen measurements on different thicknesses are shown on the left side with an inset schematic of the experiment. The figure on the right shows the analysis of the measurements. Data points were combined to guide the eye and error bars are large because of the finite thickness effect discussed in the text.

This means, that comparatively thick multilayers have to be fabricated with thicknesses between $500 \mathrm{~nm}$ and $1 \mu \mathrm{m}$ out of very thin layers for a high interface density. In this way multilayers with up to 800 individual nanoscale layers have been fabricated with PLD, which is a highly non-trivial endeavor. In order to achieve a sufficiently high accuracy, a precise control of the laser deposition process with both, laser energy corrections and laser pulse corrections is necessary.

The second approximation has been done concerning heat losses. They may occur at different stages during the TTR measurement, mainly as radiation at the surface, losses in the $\mathrm{Cu}$-layer and radial heat transport in the multilayer. The effect of radiative heat losses can be described as Boltzmann heat radiation with the following formula:

$$
P=\epsilon(T) \sigma A T^{4}
$$

Here, $P$ is the power of the emitted radiation, $\epsilon(T)$ the emissivity of $\mathrm{Cu}$, which accounts for the fact that $\mathrm{Cu}$ is no black body, $\sigma$ is the Stefan-Boltzmann constant, $A$ is the area and $T$ the temperature. These heat losses are comparatively small and can be estimated using appropriate values for $\epsilon, \sigma, A$ and $T$. First, the emissivity of $\mathrm{Cu}$ has a value around $\epsilon_{\mathrm{C} u} \approx 0.015$ depending on the temperature and is thereby much smaller than the value of $\epsilon=1$ for a black body. Second, the StefanBoltzmann constant is a very small number, namely $\sigma \approx 5.7 \cdot 10^{-8} \mathrm{~W} /\left(\mathrm{m}^{2} \mathrm{~K}^{4}\right)$. 
Taking an area of ca. $2 \mathrm{~cm}^{2}$ and a temperature of ca. $500 \mathrm{~K}$ into account, the radiative losses are approximately $P \approx 10 \mathrm{~mW}$. Taking the same assumptions for heat conduction through a thin film multilayer with a thermal conductivity of only $0.1 \mathrm{~W} / \mathrm{mK}$ and an overall thickness of $1 \mu \mathrm{m}$, the conductive power is $P_{\text {cond }} \approx 0.1 \mathrm{~W} / \mathrm{mK} \cdot 2 \mathrm{~cm}^{2} \cdot 200 \mathrm{~K} / 1 \mu \mathrm{m} \approx 4000 \mathrm{~W}$ and as such, several times of magnitude higher than the radiative losses. In order to compare the conductive lateral losses in the $\mathrm{Cu}$ top layer with the conducted cross-plane heat, it is sufficient to compare the area through which the conduction takes place. Cross-plane is approximately $2 \mathrm{~cm}^{2}$ as stated above, which can be seen as a circle with a radius of ca. $r \approx 0.8 \mathrm{~cm}$. That radius encompasses a circle with a circumference of $U \approx 5 \mathrm{~cm}$. Since the layer thickness of the $\mathrm{Cu}$ is only about $d \approx 50 \mathrm{~nm}$, the total lateral area of the $\mathrm{Cu}$ is only $A_{\mathrm{Cu}} \approx 2.5 \cdot 10^{-5} \mathrm{~cm}^{2}$ again several orders of magnitude smaller than the cross-plane area. The same calculation can be used in order to estimate lateral losses during the conduction through the multilayer. Here, a thickness of ca $1 \mu \mathrm{m}$ is better suited in order to compare the conductive areas, which still results only in a value of $A_{M L} \approx 5 \cdot 10^{-4} \mathrm{~cm}^{2}$, which is again much smaller than the cross-plane area of heat conduction. The difference is such big that even in the absolutely unlikely case of a material with a thermal conductivity that is ten times higher in lateral direction through the layers compared to the cross-plane case, the approximation would still be valid.

The greatest source of heat loss arises when comparing the lateral conduction through $\mathrm{Cu}$ with the cross-plane conduction through a very insulting material. Taking into account a thermal conductivity of $\kappa_{C u}=400 \mathrm{~W} / \mathrm{mK}$ for $\mathrm{Cu}$ and a value of $\kappa_{M L}=0.1 \mathrm{~W} / \mathrm{mK}$ for the multilayer, the energy conduction throught he multilayer is still ten times higher than through the $\mathrm{Cu}$ top layer.

All in all, this explains the validity of the applied approximations for TTRmeasurements of a certain material even with low thermal conductivities. In order to measure thin film multilayer, which is very important for this work and will be explained in chapter 6, the compound material beneath the $\mathrm{Cu}$ layer has to be modeled as a quasi-material with a combined heat capacity, a combined density and an overall thermal conductivity. For a description of the thermal conductivity the diffusive regime is chosen that allows to express the overall crossplane thermal conductivity via the values of the individual components as well as a boundary resistance, as explained already beforehand in this chapter. The according implementation will be subject of chapter 5 .

The assumption of mainly diffusive heat transport arises from the fact, that the 
multilayers presented here have roughnesses in the range of $0.3 \mathrm{~nm}$ to $0.8 \mathrm{~nm}$ which is large enough to destroy coherence of phonons in a wide frequency range [186]. Moreover, the crossover from diffusive to coherent transport lies by interface densities of around $1 \mathrm{~nm}^{-1}$ which is higher than the interface densities presented in this thesis that achieved values for one W/PC multilayer sample in chapter 7 up to $0.6 \mathrm{~nm}^{-1}$ [163]. All other samples in this thesis have even lower interface densities making diffusive transport clearly the dominant mechanism in thermal conductivity in this thesis.

Altogether, the TTR method presented here, which was implemented in this work, proved to measure successfully thermal conductivities of various bulk materials as well as multilayers and will be of great importance for most of the upcoming chapters. 


\section{Phonon localization in ultrathin layered structures}

F. Döring ${ }^{1}$, C. Eberl ${ }^{1}$, S. Schlenkrich ${ }^{1}$, F. Schlenkrich ${ }^{1}$, S. Hoffmann ${ }^{1}$, T. Liese ${ }^{1}$, H. U. Krebs ${ }^{1}$, S. Pisana ${ }^{2 *}$, T. Santos ${ }^{2}$, H. Schuhmann ${ }^{3}$, M. Seibt ${ }^{3}$, M. Mansurova ${ }^{4}$, H. Ulrichs $^{4}$, V. Zbarsky ${ }^{4}$ and M. Münzenberg ${ }^{4}$

1 Institut für Materialphysik, Georg-August-University Göttingen, FriedrichHund-Platz 1, 37077 Göttingen, Germany

2 San Jose Research Center, HGST a Western Digital Company, 3403 Yerba Buena Road, San Jose CA 95135, USA

3 IV. Physikalisches Institut, Georg-August-University Göttingen, Friedrich-HundPlatz 1, 37077 Göttingen

4 I. Physikalisches Institut, Georg-August-University Göttingen, Friedrich-HundPlatz 1, 37077 Göttingen, and Institute of Physics, Ernst-Moritz-Arndt University Greifswald, Germany

* Current address: Department of Electrical Engineering \& Computer Science, York University, 4700 Keele Street, Toronto ON M3J 1P3, Canada

Applied Physics A 119:11-18 (2015)

doi:10.1007/s00339-015-9037-z

Accepted for publication on 4 February 2015

Published online 12 February 2015

\subsection{Abstract}

An efficient way for minimizing phonon thermal conductivity in solids is to nanostructure them by means of reduced phonon mean free path, phonon 
scattering and phonon reflection at interfaces. A sophisticated approach towards this lies in the fabrication of thin multilayer films of different materials. In this paper we show by femtosecond-pump-probe reflectivity measurements that in different multilayer systems with varying acoustic mismatch (consisting of metals, semiconductors, oxides and polymers) oscillations due to phonon localization can be observed. For the growth of multilayer films with well-defined layer thicknesses we used magnetron sputtering, evaporation and pulsed laser deposition. By altering the material combinations and reducing the layer thicknesses down to $3 \mathrm{~nm}$, we observed different mechanisms of phonon blocking, reaching in the frequency regime up to $360 \mathrm{GHz}$.

\subsection{Introduction}

Multilayer insulation has been widely used to control the heat flow in turbine coatings and cryogenic applications [187]. Depending on the system and its structure and microstructure, such thermal processes take place on a time scale of up to some ns (for metals) or ms (for oxides), and can be studied in multilayer structures down to the nm-scale $[188,189]$. Results on the minimal thermal conductivity of solids were summarized by Goodson [35]. The highest thermal conductivity is generally achieved through crystalline order, while it is much lower in amorphous materials such as oxides, polymers or porous materials. Multilayers of disordered materials lead to further conductivity reductions, with both disorder and interface blocking of energy transfer working together.

Using $\mathrm{W} / \mathrm{Al}_{2} \mathrm{O}_{3}$ multilayers, it was shown that an increasing number of interfaces strongly reduces the thermal conductivity even below the amorphous limit [39]. This result suggests that high densities of interfaces between dissimilar materials may provide a route for the production of thermal barriers with ultra-low thermal conductivity. A mismatch between the acoustic impedances of the adjacent materials at the interface prohibits phonon propagation across (so-called "phonon blocking"). Ultralow conductivity was actually observed in a layered, fully dense solid $\mathrm{WSe}_{2}$ [142]. Due to its natural layered structure, it has the maximum number of interfaces possible in solid state materials. Because in this structure the bonding type is very different within the atomic layer planes and across the planes of its layered structure, this phonon frequency mismatch results in a disturbance in the phonon propagation perpendicular to the layers. 
The phonon frequency mismatch at interfaces depends on the material combinations used and should be strongest in hard/soft materials as for instance metal/polymer layered structures. Such systems have not been studied much so far, probably due to difficulties in growing them with small layer thicknesses and high quality interfaces. While in crystalline films it can be assumed that the thermal conductivity is already significantly reduced compared to the bulk value due to grain boundaries [84], extremely low thermal conductivity should be obtained as soon as phonon wave localization due to reflection at interfaces with large acoustic mismatch occurs.

For the study of phonon localization, fs-pump-probe experiments are the method of choice, because this technique yields microscopic insights into the early processes after laser excitation with its unique time resolution given by the laser pulse lenght [39,142,190-193]. For instance, the thermal relaxation of the electronic distribution after non-equilibrium electron heating was studied in different metal thin films. On the time scale of the electron-phonon coupling time, the reflectivity line shape demonstrates the generation of non-equilibrium electron temperatures which cool to the lattice on a ps time scale. Thus, the ultrafast heating ultimately generates phonons [193].

Consider now longitudinal phonons (with frequency $f$, wave number $k$ ) travelling from the surface of an acoustically hard material towards an interface of an acoustically soft material. Transmission of acoustic waves through such a onedimensional system can be described by the transfer-matrix approach, which is well-known from optics. Upon traversing the hard layer, the wave acquires a phase $\phi=k D a=2 \pi f d_{A} / v_{A}$, where $d_{A}$ is the thickness of the layer and $v_{A}$ the group velocity. When impinging the interface towards the second, soft material, a small part of the wave will be transmitted, the other part reflected. When restricted to perfectly normal incidence, the acoustic mismatch model expresses e.g. the amplitude coefficient for the forward transmission $t_{A B}=2 Z_{A} /\left(Z_{A}+Z_{B}\right)$ and backward reflection $r_{A B}=\left(Z_{A}-Z_{B}\right) /\left(Z_{A}+Z_{B}\right)$ via the acoustic impedance $Z=\rho v$, where $\rho$ is the mass density [194]. Generally, in each layer one forward and one backward propagating partial wave may exist at a given frequency. Their complex amplitudes at different positions can be related via transfer matrices $T(f)$, whose components allow to compute the reflectivity $R(f)$ [194]. Note that a large impedance mismatch implies a strong decoupling of the hard from the soft layer. For certain frequencies, a standing wave is formed in one of the constituent layers or in the whole stack. In periodic multilayer systems, only Bloch-like modes 
are allowed, and band gaps open up, where propagation is forbidden. In turn, the existence of band gaps enables localized surface modes, whose frequencies are in the gap but which are strongly damped perpendicular to the surface [195]. For $\mathrm{nm}$ thick films, the relevant phonon frequencies are expected to lie in the range of several 10 to a few $100 \mathrm{GHz}$. Therefore, coherent surface reflectivity oscillations on a ps timescale, indicating standing or localized phonon waves, should be observable in pump-probe experiments performed on multilayers with small layer thicknesses.

Thus, the aim of this paper is to study which multilayer systems lead to observable localized phonon modes. To this end we used the potential of magnetron sputtering, evaporation, and pulsed laser deposition (PLD) allowing the growth of combinations of different material classes like metals, semiconductors, oxides and polymers with well-defined layer thicknesses. The phonon localization is studied in fs-pump-probe reflectivity measurements by probing surface vibrations after fs-laser excitation. We will see that standing phonon waves that are reflected by adjacent interfaces and therefore localized within specific layers, as well as strongly damped surface modes, can be observed at GHz frequencies .

\subsection{Experimental}

For the deposition of $\mathrm{W} / \mathrm{Si}$ and $\mathrm{PC} / \mathrm{Cu}$ (PC: polycarbonate) layered structures, PLD was realized by using a $\mathrm{KrF}$ excimer laser (wavelength of $248 \mathrm{~nm}$, pulse duration of $30 \mathrm{~ns}$, repetition rate of $10 \mathrm{~Hz}$ ) as described earlier [52]. The films were grown at room temperature in ultrahigh vacuum of about $10^{-8}$ mbar at a target-to-substrate distance of $7 \mathrm{~cm}$. For metals and oxides a laser fluence of $1-5 \mathrm{~J} / \mathrm{cm}^{2}$ was used, but in the case of polymer deposition a much lower fluence of $70 \mathrm{~mJ} / \mathrm{cm}^{2}$ had to be taken to preserve the structure. FePtAgCu/MgO/NiTa and $\mathrm{CoFeB} / \mathrm{MgO}$ multilayers were deposited by magnetron sputtering and e-beam evaporation in ultrahigh vacuum of about $5 \cdot 10^{-10}$ mbar.

The deposition rates of the components were determined by profilometry (Dektak 150, Veeco), in-situ rate monitoring (Inficon SQM 160) and X-ray reflectivity (XRR). The X-ray measurements were carried out by a Phillips X'Pert diffractometer with CoK $\alpha$-radiation $(\lambda=0.179 \mathrm{~nm})$ and were analyzed with the software IMD [196]. For further analysis, transmission electron microscopy (TEM) images were taken by 
using a Philips CM30 and for high-resolution a CM 200 with an image aberration corrector. The fs-pump-probe experiments were performed using exciting laser pulses of a $\mathrm{Ti}_{2} \mathrm{Al}_{2} \mathrm{O}_{3}$ laser with a pulse duration of $40 \mathrm{fs}$, photon energy of $1.55 \mathrm{eV}$ and of $10-40 \mathrm{~mJ} / \mathrm{cm}^{2}$ from a RegA 9050I regenerative amplifier system (repetition rate $250 \mathrm{kHz}$ ).

\subsection{Results and discussion}

First we show that high quality multilayers consisting of different kinds of material combinations with well-adjusted single layer thicknesses and controllable interface and surface roughness could be grown by PLD. In Fig. 3.1, cross-sectional TEM images of some examples of the produced layered structures can be seen. For the ongoing investigations with the fs-pump-probe reflectivity method, especially the $\mathrm{W} / \mathrm{Si}$ and $\mathrm{PC} / \mathrm{Cu}$ systems are of large interest, since they feature a very high acoustic mismatch. In both cases, multilayers with sharp interfaces could be obtained. For the deposition of $\mathrm{W}$ and $\mathrm{Si}$, different laser fluences were used because of the large difference in their melting points. Laser fluences of $3.3 \mathrm{~J} / \mathrm{cm}^{2}$ and $1.7 \mathrm{~J} / \mathrm{cm}^{2}$, respectively, were chosen as a compromise between low droplet formation, high deposition rate and reduced resputtering effects (for details see $[144,197])$. For the fabrication of the PC/Cu multilayers, a laser fluence of $2.1 \mathrm{~J} / \mathrm{cm}^{2}$ has been chosen for $\mathrm{Cu}$ but a much lower laser fluence of $70 \mathrm{~mJ} / \mathrm{cm}^{2}$ (close to the deposition threshold) had to be taken for PC to preserve the polymer composition. We found, that laser deposited PC has an elastic modulus of $E \approx 6 \mathrm{GPa}$ (not shown here) and is therefore, as a rather hard polymer, a suitable candidate for smooth polymer/metal multilayers. These $\mathrm{PC} / \mathrm{Cu}$ multilayer structures are especially interesting for thermal insulation since polymers have an intrinsic thermal conduction that is even lower than that of oxides (see [35]) and the acoustic mismatch between polymers and metals is very high.

In addition, also other multilayers, representative for an oxide/oxide $\left(\mathrm{MgO} / \mathrm{ZrO}_{2}\right)$ and a metal/oxide $\left(\mathrm{Ti} / \mathrm{ZrO}_{2}\right)$ system were laser deposited. Again, the cross-section TEM images of Fig. 3.1 indicate sharp interfaces for both material combinations. Due to the amorphous structure of at least one material, both multilayers systems should show a low thermal conductivity $[35,39,84]$ because of phonon blocking, especially, when the number of interfaces is increased. 


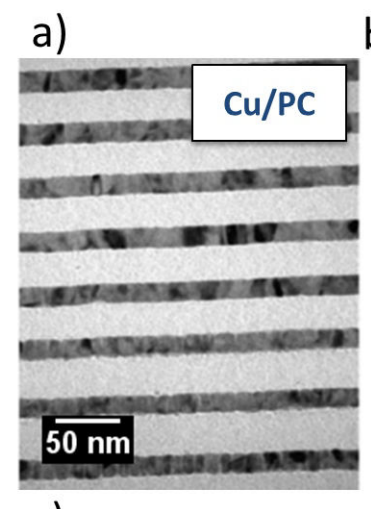

c)

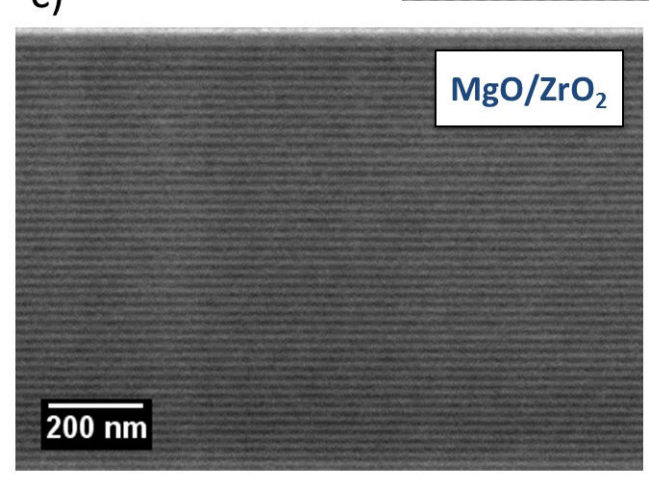

b)
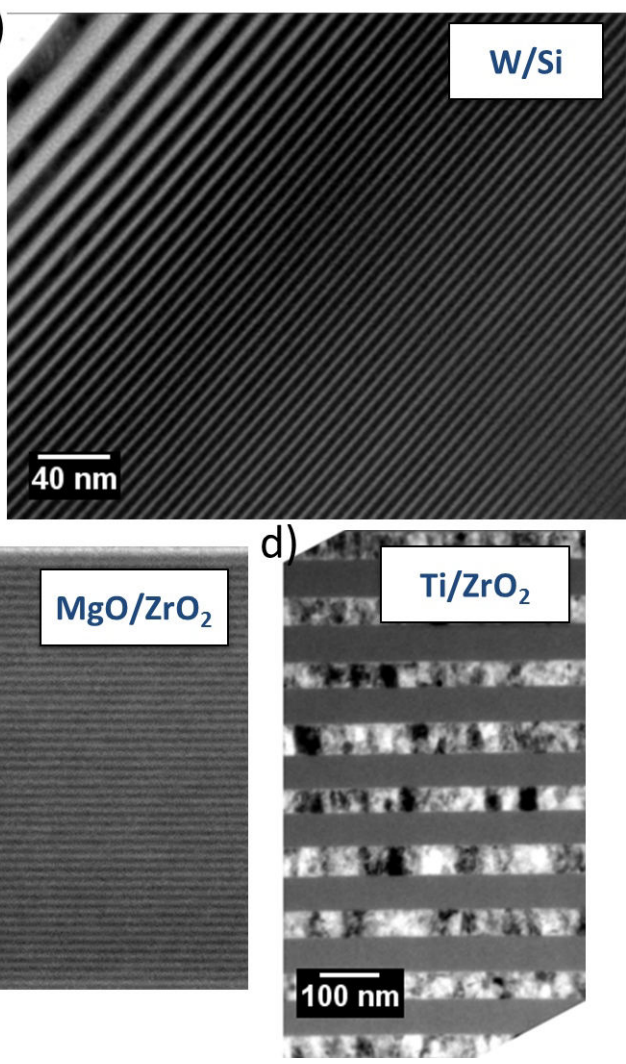

Figure 3.1.: Cross-sectional TEM micrographs of laser deposited multilayer structures of the systems a) metal/polymer ( $\mathrm{Cu} / \mathrm{PC}), \mathrm{b})$ metal/semiconductor $(\mathrm{W} / \mathrm{Si}), \mathrm{c})$ oxide/oxide $\left(\mathrm{MgO} / \mathrm{ZrO}_{2}\right)$, and d) metal/oxide $\left(\mathrm{Ti} / \mathrm{ZrO}_{2}\right)$

In the next series of experiments, we systematically reduced the layer thicknesses in the $\mathrm{Ti} / \mathrm{ZrO}_{2}$ system and studied the layer thickness limits for which multilayers with sharp interfaces could be obtained. For simplicity, the multilayers were analyzed by X-ray reflectivity measurements (XRR), where strong Kiessig fringes together with sharp Bragg peaks are clear indications for smooth surfaces and layered periodic structures. In Fig. 3.2, the reflectivity measurements and corresponding simulations using IMD software [196] are depicted for the two $\mathrm{Ti} / \mathrm{ZrO}_{2}$ multilayers with smallest layer thicknesses.

As can be followed directly from the X-ray diffraction patterns, multilayers with layer thicknesses of below $2 \mathrm{~nm}$ still display sharp Bragg peaks, indicating periodically layered structures. As can be seen, the decrease of the reflectivity with scattering angle, the Kiessig fringes as well as the Bragg reflections can be reproduced by the corresponding simulations with high accuracy. IMD [196] simulations of the spectra (included in Fig. 3.2 in red) result in typical interface roughnesses of below $0.5 \mathrm{~nm}$. This low roughness of the pulsed laser deposited 

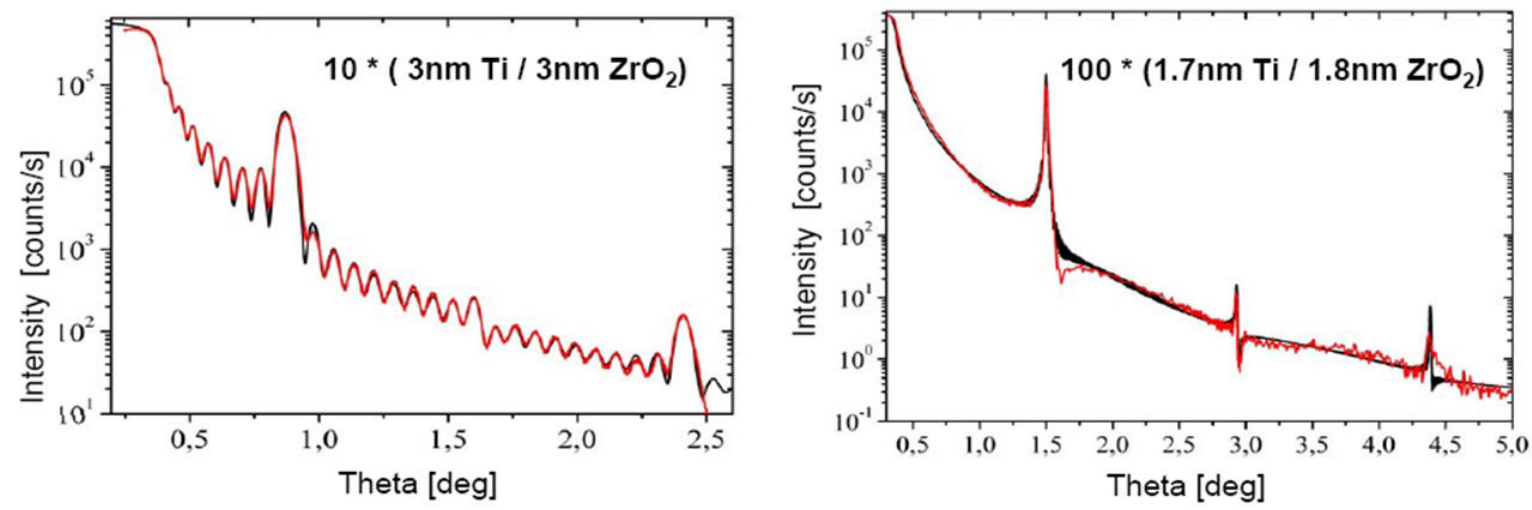

Figure 3.2.: XRR measurements (with IMD-simulations in red) of $\mathrm{Ti} / \mathrm{ZrO} \mathrm{CO}_{2}$ multilayers with very low layer thicknesses.

films arises from the high energy of deposited particles (typically in the order of $100 \mathrm{eV}$ ) [145] leading also for thicker films to the effect of cumulative smoothening $[198,199]$. Such a low interface roughness is necessary in order to apply the acoustic mismatch model for the formation of standing phonon waves. To complete the set of material combinations, two further combinations of complex metal/metal and metal/oxide systems (FePtAgCu/MgO/NiTa and $\mathrm{CoFeB} / \mathrm{MgO}$, respectively) were grown by magnetron sputtering ( $\mathrm{FePtAgCu}, \mathrm{NiTa}, \mathrm{CoFeB}$ ) and e-beam evaporation $(\mathrm{MgO})$. In the case of $\mathrm{CoFeB} / \mathrm{MgO}$-multilayers, a crosssectional high-resolution TEM micrograph for a multilayer with layer thicknesses of about $3 \mathrm{~nm}$ is depicted in Fig. 3.3. It can clearly be seen that the metallic CoFeB grows amorphous, while even the individual layers of the nanocrystalline oxide grains can be resolved as $\mathrm{MgO}$ with [001]-texture. Thus, also this is a further suitable system for phonon blocking and localization.

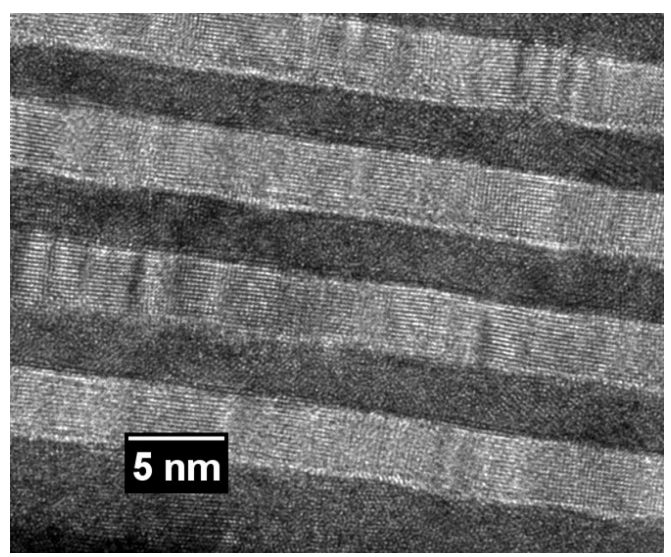

Figure 3.3.: High-resolution cross-sectional TEM micrographs of sputtered $\mathrm{CoFeB} / \mathrm{MgO}$ multilayers with layer thicknesses of $3.2 \mathrm{~nm}$. 
From the wide range of layered structures of different materials combinations with sharp interfaces, some systems with different strong acoustic mismatch were selected and specially designed for fs-pump-probe reflectivity measurements, to study whether phonon localization within the layered structures can be obtained. In the first experiment we chose a simple $\mathrm{PC} / \mathrm{Cu}$ bilayer with very high acoustic mismatch between the hard metal layer $\left(Z_{C u}=42 \mathrm{MPa} / \mathrm{m}\right)$ and the soft polymer $\left(Z_{P C}=3 \mathrm{MPas} / \mathrm{m}\right)$, deposited on a $S i$ substrate $\left(Z_{S i}=20 \mathrm{MPas} / \mathrm{m}\right)$. In this system, due to the isolating properties of the polymer, only phonon but no electron transport can take place across the metal/polymer interface. The idea was to excite the thin $\mathrm{Cu}$ layer $(d=20 \mathrm{~nm})$ by a laser pulse, and to study how the arising phonons are transferred into and transported within the $100 \mathrm{~nm}$ thick PC layer. The surface excitation was done using a laser fluence of $10 \mathrm{~mJ} / \mathrm{cm}^{2}$ and the surface reflectivity dynamics was probed after different delay times. The laser energy is absorbed by the electron system, which successively equilibrates its temperature with the phonon system within the electron-phonon-equilibration time of a few ps [200]. Thereafter, the thermal relaxation takes place on a ns time scale. The excitation of phonons can be probed with the probe beam, because the stress associated with the excited acoustic phonons changes the reflectivity of the surface. The time dependent reflectivity measurements after laser excitation are depicted in Fig. 3.4(a). Within the measurement of the surface reflectivity, clear oscillations can be observed during thermal relaxation. These oscillations let us expect that a stress wave, initially excited in the metal layer due to surface heating, forms a standing wave within the PC by reflection at the adjacent $\mathrm{Cu}$ and $\mathrm{Si}$ interfaces. As can be seen in the Fourier transform of the data (Fig. 3.4(b)), these oscillations correspond to a frequency at $f_{0}=38 \mathrm{GHz}$.

Since in the experiment only information from the surface can be obtained, a finite-difference time-domain (FDTD) numerical simulation [201] was conducted in order to determine the mode profile $A\left(f_{0}, z\right)$, by taking the layer thicknesses of the used $\mathrm{Cu} / \mathrm{PC}$ bilayer and typical material parameters into account. To this end, a sinusoidal stress was applied to the surface, and the frequency dependence of the response of the system was determined. Similar to the experiments, a resonance-like behavior around $f_{0}$ was found. The mode profile associated with this resonance indicates the occurrence of a standing wave inside the PC, as expected (depicted in Fig. 3.4 (c)).

As a second example, a special nonperiodic multilayer, compromising a $7 \mathrm{~nm}$ thick $\mathrm{FePtAgCu}$ layer was chosen. Such systems are under investigation within the 
(a)

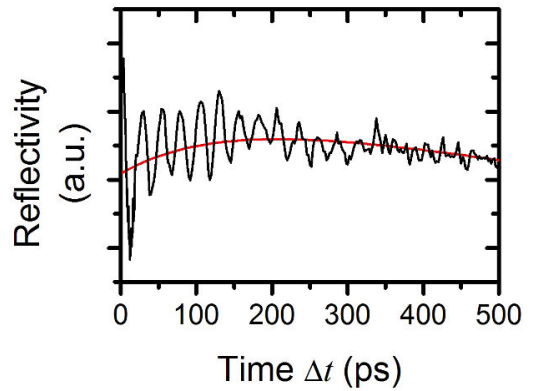

(d)

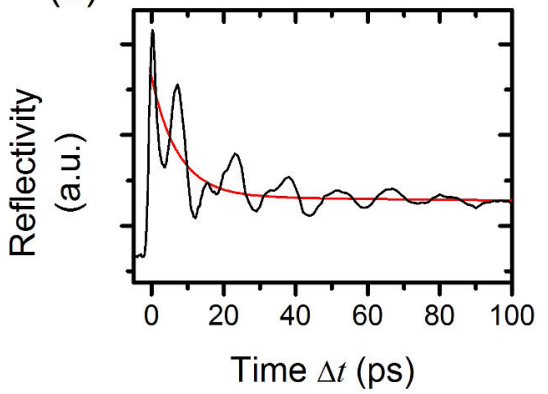

(b)

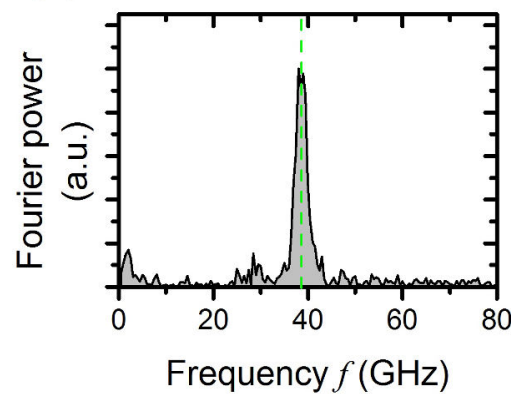

(e)

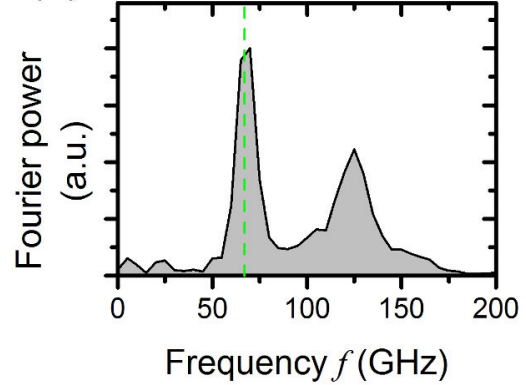

(c)

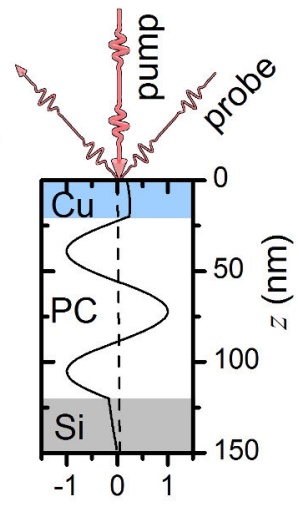

Mode profile $A\left(f_{0}\right)$

(f)

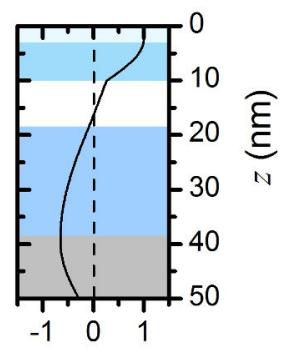

Mode profile $A\left(f_{0}\right)$

Figure 3.4.: (a) Change of surface reflectivity plotted against delay time after laser excitation. The decaying background of incoherent heating has been removed from the signal to extract the coherent phonon part. (b) Fourier power spectrum of the reflectivity oscillations indicating a localized standing phonon mode at $38 \mathrm{GHz}$ in the $100 \mathrm{~nm}$ PC layer. (c) Sketch of the pump-probe reflectivity experiment on the $\mathrm{Cu} / \mathrm{PC}$ bilayer on a $\mathrm{Si}$ substrate together with the mode profile determined by a FDTD simulation. (d-f) Same graphs for the heat assisted magnetic recording (HAMR) FePtAgCu/MgO/NiTa sample. (d) The continuous non-oscillating curve (red) models a background of incoherent phonons and electronic excitations, which was subtracted prior to computing the Fourier spectrum shown in (e). Here, the dashed vertical line marks the frequency $f_{0}$ of the dominant phonon mode. (f) FDTD calculation of the mode profile corresponding to $f_{0}$.

context of novel concepts for nonvolatile data storage. The emerging technique of heat assisted magnetic recording (HAMR) combines magnetic data storage with laser heating, in order to temporarily decrease the energy barrier which has to be overcome to write a magnetic bit. Here, understanding and tailoring of heat flow can help to optimize the efficiency of the writing process. In the pump-probe experiments, the reduced layer thicknesses, as compared to the $\mathrm{Cu}$-PC sample, should lead to an increase of the oscillation frequencies in the reflectivity measurements. This is indeed the case as can be followed in Fig. 3.4 (d, e) by the reduced time between the oscillations in the reflectivity dynamics. Due 
to the more complex structure, in the Fourier transform two dominant phonon modes, at $f_{0}=67 \mathrm{GHz}$ and $125 \mathrm{GHz}$, occur. The wave profile of the mode at $67 \mathrm{GHz}$ is shown in Fig. 3.4 (f), again determined by the same FDTD method as discussed before.

The results obtained in the PC/Cu-bilayer and HAMR multilayer show that in both systems strong oscillations occur in the reflectivity measurements, whose frequency can be tuned by the thickness of the used layered structures. These oscillations imply strong reflections at interfaces and the formation of standing phonon waves. As deduced from the simulations, the impedance mismatch can be exploited as a mechanism responsible for the reflections and the formation of standing waves.

In the following we turn to whether such phonon oscillations can also be observed in periodic multilayers with low layer thicknesses, and how the results depend on the acoustic mismatch of the materials involved. In periodic multilayers, the superlattice leads to a band structure in the phonon spectra, implying the occurrence of gaps. Thus, it was also interesting to study whether the frequencies of the optically excited phonons lie within the bands or in between in the gaps. This should have strong influence on the transport of the phonons within the multilayer, as frequencies within the band gaps should be hindered in their transport. For these experiments, two types of periodic multilayers with five periods each were chosen, namely $\mathrm{CoFeB}(3.15 \mathrm{~nm}) / \mathrm{MgO}(3.15 \mathrm{~nm})$ and $\mathrm{W}(12 \mathrm{~nm}) / \mathrm{Si}(7 \mathrm{~nm})$. While for the first multilayer a very small acoustic mismatch exists $\left(Z_{\mathrm{CoFeB}}=39 \mathrm{MPas} / \mathrm{m}\right.$, $\left.Z_{\mathrm{MgO}}=35 \mathrm{MPas} / \mathrm{m}\right)$, the second multilayer has a large acoustic mismatch $\left(Z_{W}=101 \mathrm{MPas} / \mathrm{m}, Z_{S i}=20 \mathrm{MPas} / \mathrm{m}\right)$.

The experimental reflectivity data of the $\mathrm{CoFeB} / \mathrm{MgO}$ multilayer with very low impedance mismatch is shown in Fig. 3.5 (a), and the corresponding Fourier spectrum in Fig. 3.5 (b). Obviously, in this multilayer the reflectivity signal is comprised of multiple phonon modes, as can already be seen in the complex oscillations in the reflectivity measurements. In order to classify the mode profile and the associated power spectra into a band structure view, transfermatrix calculations for longitudinal waves were performed as mentioned in the introduction. The coefficient of reflection $R(f)$ (note that a large $R$ corresponds to a band gap) was determined, which is also shown in Fig. 3.5 (b). Compared with the band structure, determined from the transfer matrix calculations, one can follow that the oscillation frequencies predominantly lie at the upper end of the first frequency band. Theoretically, a resonance-like mode can be expected there. 
This means that transport of stress waves along the multilayer structure can take place indicating a standing wave in the complete stack. Considering for example the largest peak in the Fourier spectrum at $f_{0}=360 \mathrm{GHz}$, the FDTD calculations relate the wave profile of a higher order collective resonance mode to this peak.

(a)

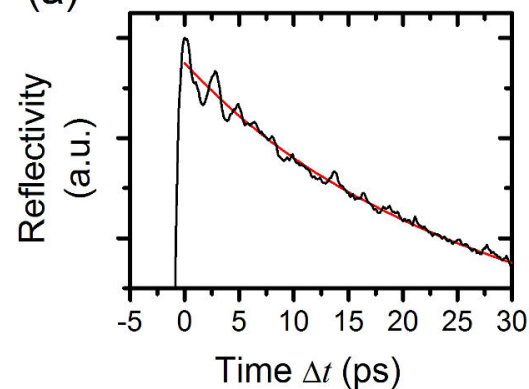

(d)

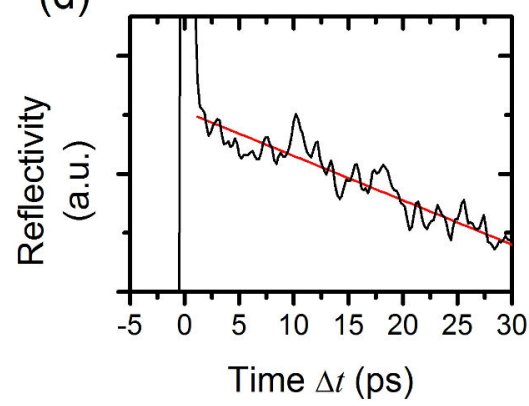

(b)

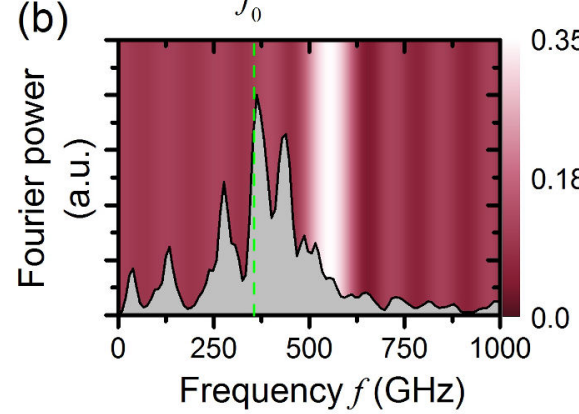

(e)

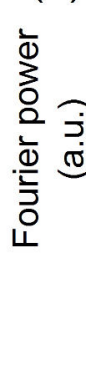

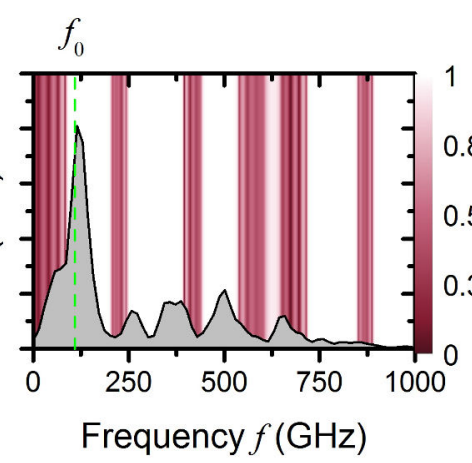

(c)

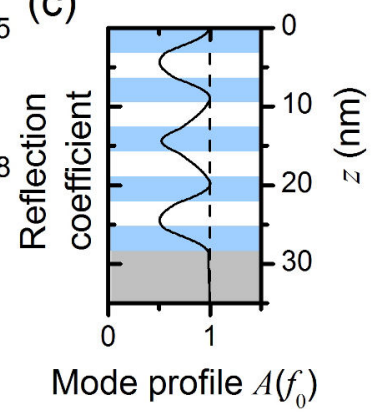

(f)

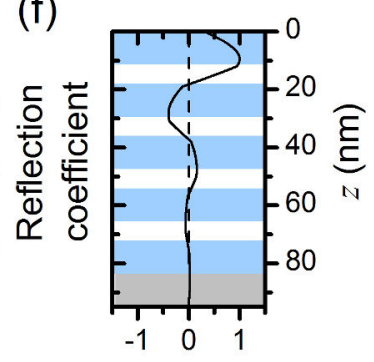

Mode profile $A\left(f_{0}\right)$

Figure 3.5.: Results of the pump-probe reflectivity experiment on (a) a CoFeB $(3.15 \mathrm{~nm}) / \mathrm{MgO}(3.15 \mathrm{~nm})$ multilayer sample. Again with the background in red, that was removed prior to computing the Fourier spectrum depicted in (b). Here the Fourier spectrum is underlaid by a transfer-matrix calculation of the reflection coefficient $R(f)$ for longitudinal waves. (c) FDTD calculation of the in-plane mode profile showing a localized standing wave within the layer structure. (d) equally obtained reflectivity results of a $\mathrm{W}(12 \mathrm{~nm}) / \mathrm{Si}(7 \mathrm{~nm})$ multilayer sample with (b) corresponding Fourier power underlaid with a calculated band structure. Note that the majority of modes are lying in the band gaps leading to (f) a mode profile of sagittal wave with a damped oscillation inside the multilayer due to the high acoustic mismatch.

In contrast, in the W-Si system with large acoustic mismatch, band gaps cover a large range of the experimentally accessible frequencies (see Fig. 3.5 (e)), as again determined by transfer matrix calculations. Again, oscillations can be seen in the reflectivity measurements. But in this system, most of the excited modes have frequencies located in the gaps. Thus, the nature of these modes must be different than the nature of the modes discussed previously. Indeed, a good agreement with 
the experiment can be found by modelling the observed modes as higher order sagittal elastic waves [195]. Such modes are localized at the surface, and in general propagate parallel to the surface. For the case relevant here $k_{\|}=0$, this mode is polarized parallel to the surface normal, and thus can be excited by a normally incident laser pulse. By extending the transfer-matrix approach to such waves, the mode profile corresponding to the dominant mode at frequency $f_{0}=109 \mathrm{GHz}$ shown in Fig. 3.5(f) was computed from standard material parameters. It can clearly be seen, that the propagation of the stress wave is strongly attenuated along the growth direction of the multilayer. Thus, after being excited at the surface, this wave hardly reaches the substrate.

\subsection{Conclusion}

In summary, we could show that phonon localization and standing wave formation can be observed in many material combinations by time-resolved pump-probe measurements. These experiments were facilitated by the fact that layered structures of metals, semiconductors, oxides and polymers could be grown with highly accurate layer thickness and low interface roughness by sputtering, evaporation and PLD, respectively. The use of alternating material properties (for instance hard/soft and crystalline/amorphous combinations) allows producing standing phonon waves in layered structures which can be one way to strongly reduce the thermal heat flow across multilayered structures. In these first experiments, we already could demonstrate phonon standing waves tunable by the layer thicknesses over a wide range with frequencies up to $360 \mathrm{GHz}$. This demonstrates that with lowering the layer thicknesses even frequencies above $1 \mathrm{THz}$ could be reached. Furthermore it can be concluded that phonon transport and with it the thermal energy transport $\Delta Q$ can be hindered at the interfaces by a large acoustic mismatch of the materials used (phonon blocking). Thus, the results on the bilayers and multilayers prove that certain phonon modes are blocked for phonon propagation at frequencies in the $\mathrm{GHz}$ region. As a consequence the energy transfer across the multilayer can be suppressed by design (material combination and layer thicknesses). Our ongoing research will concentrate on metal/PC multilayers due to their strong acoustic mismatch, and on multilayers of high-melting point metals and oxides for potential thermal barrier coatings that 
block high energy phonons and hot electron heat transfer.

\section{Acknowledgment}

This work was supported by the Deutsche Forschungsgemeinschaft (SFB 1073). 



\section{Confinement of phonon propagation in laser deposited Tungsten/Polycarbonate multilayers}

Florian Döring ${ }^{1}$, Henning Ulrichs ${ }^{2}$, Sinja Pagel ${ }^{1}$, Markus Müller ${ }^{2}$, Maria Mansurova $^{2}$, Matthias Müller ${ }^{3}$, Christian Eberl ${ }^{1}$, Torben Erichsen ${ }^{1}$, Dennis Huebner ${ }^{4}$, Philipp Vana ${ }^{4}$, Klaus Mann ${ }^{3}$, Markus Münzenberg ${ }^{5}$, Hans-Ulrich Krebs ${ }^{1}$

1 Institute for Materials Physics, Georg-August University Göttingen, FriedrichHund-Platz 1, 37077 Göttingen, Germany

2 1st Institute of Physics, Georg-August University Göttingen, Friedrich-HundPlatz 1, 37077 Göttingen, Germany

3 Laser-Laboratorium Göttingen e.V., Hans-Adolf-Krebs-Weg 1, 37077 Göttingen, Germany

4 Institute of Physical Chemistry, Georg-August-University Göttingen, Tammannstrasse 6, 37077 Göttingen, Germany

5 Institute of Physics, Ernst-Moritz-Arndt University Greifswald, Felix-HausdorffStr. 6, 17487 Greifswald, Germany

New Journal of Physics 18, 092002, pp 1-9 (2016)

doi: 10.1088/1367-2630/18/9/092002

Accepted for publication on 19 September 2016

Published online 29 September 2016 as Open Access Fast Track Communication 


\section{Confinement of phonon propagation in laser deposited Tungsten/Polycarbonate}

multilayers

\subsection{Abstract}

Nanoscale multilayer thin films of W and PC (Polycarbonate) show, due to the great difference of the components' characteristics, fascinating properties for a variety of possible applications and provide an interesting research field, but are hard to fabricate with low layer thicknesses. Because of the great acoustic mismatch between the two materials, such nanoscale structures are promising candidates for new phononic materials, where phonon propagation is strongly reduced. In this article we show for the first time that W/PC-multilayers can indeed be grown with high quality by pulsed laser deposition. We analyzed the polymer properties depending on the laser fluence used for deposition, which enabled us to find best experimental conditions for the fabrication of high-acousticmismatch W/PC multilayers. The multilayers were analyzed by fs pump-probe spectroscopy showing that phonon dynamics on the ps time-scale can strongly be tailored by structural design. While already periodic multilayers exhibit strong phonon localization, especially aperiodic structures present outstandingly low phonon propagation properties making such 1D-layered W/PC nano-structures interesting for new phononic applications.

\subsection{Introduction}

Multilayer thin films of metals and polymers found their way into a variety of applications and are a prominent research field for further improvement [57, 202-204]. Especially, the totally different material properties of the two components allow tuning of optical, mechanical, electrical and acoustic characteristics [75, 205-208]. For the field of acoustics, such nanoscale multilayers are of special interest because of the high difference in density and sound velocity of the two components leading to a high acoustic mismatch at the interfaces. Thereby, the phonon propagation probability and phonon mean free path is strongly reduced, making such nanoscale structures possible candidates for phonon blocking materials [86,209], within which also the thermal conductivity could be strongly decreased [39,142]. To improve the outstanding acoustic properties of such material combinations, the difference of the properties of the single components has to be maximized. That makes it essential to choose hard and heavy metals together with soft and lightweight materials, such as polymers $[210,211]$. For this purpose, we chose $\mathrm{W}$ with its 
very high density of $19.25 \mathrm{~g} / \mathrm{cm}^{3}$ as metal component and PC (Polycarbonate) as the corresponding low density polymer (density of about $1.2 \mathrm{~g} / \mathrm{cm}^{3}$ ), resulting in a strong acoustic mismatch and great difference of material properties. Unfortunately, the large difference in the thermal properties, e.g. melting point and thermal conductivity, makes it challenging to prepare nanoscale multilayers of those material combinations. The aim of this paper is to show that pulsed laser deposition (PLD) allows the fabrication of such nanoscale multilayers and to study the phonon propagation in these multilayers by optical fs time-resolved pumpprobe spectroscopy. To optimize the acoustic mismatch between $\mathrm{W}$ and PC, first the polymer properties had to be studied using different laser fluences during film growth. Then, the phonon dynamics were determined in a periodic and aperiodic W/PC multilayer by the fs pump-probe technique. Due to the acoustic mismatch at the interfaces, we expect little phonon propagation already for periodic structures. But, in aperiodic structures, phonon propagation through the multilayer should even be further reduced making such material combinations well suited candidates for new phononic applications $[4,212]$.

\subsection{Experimental}

For the deposition of the thin films, we used a standard pulsed $\mathrm{KrF}$ laser with $248 \mathrm{~nm}$ wavelength, $20 \mathrm{~ns}$ (FWHM) pulse duration, $5 \mathrm{~Hz}$ repetition rate, and a target-to-substrate distance of $7 \mathrm{~cm}$ [51]. All films were grown at room temperature in an ultra-high vacuum chamber at a base pressure around $10-8 \mathrm{mbar}$. Due to the fact that $\mathrm{W}$ has a melting point of $3422^{\circ} \mathrm{C}$, which is the highest for all metals [211], and a bulk thermal conductivity of $173 \mathrm{~W} / \mathrm{mK}$ [211], a high laser fluence of $3.5 \mathrm{~J} / \mathrm{cm}^{2}$ had to be taken during PLD. In contrast, PC has a glass transition temperature of around $140^{\circ} \mathrm{C}$ and a thermal conductivity of only about $0.2 \mathrm{~W} / \mathrm{mK}$ making it challenging not to destroy the polymeric structure during ablation with laser pulses at $248 \mathrm{~nm}[210,213,214]$. In order to achieve this goal, the laser is run with low energy pulses, which are damped by an attenuator. Additionally, the laser beam was defocused by a lens resulting in low laser fluences in the range from $25 \mathrm{~mJ} / \mathrm{cm}^{2}$ to $375 \mathrm{~mJ} / \mathrm{cm}^{2}$ on the target. Compared to the deposition of $\mathrm{W}$, this is a change in laser fluence of one or two orders of magnitude. In order to produce multilayers of both materials in one chamber with the same laser, a laser controlling software was written allowing to change the pulse energy during 


\section{Confinement of phonon propagation in laser deposited Tungsten/Polycarbonate} multilayers

deposition, to put a movable attenuator into the beam path (only in the case of PC) and to move the focusing lens to either focus the laser beam on $\mathrm{W}$ or defocus it on PC. The layer thicknesses of both materials could be adjusted by the number of laser pulses used for deposition. The deposition rates were measured in-situ via an oscillating quartz crystal (Inficon SQM 160) as well as ex-situ by profilometry (Dektak 150, Veeco) and X-ray reflectivity (XRR) measurements (Bruker D8 Discover with $\mathrm{Cu}-\mathrm{K} \alpha$-radiaton), which have been fitted by simulated spectra using LEPTOS 7 (Bruker). For insight into the structures, transmission electron microscopy was used (FEI, Titan). In order to get information about the structure of the polymer, near-edge X-ray absorption spectroscopy (NEXAFS) measurements have been conducted at a table-top device [215]. For measurements of hardness and roughness of the polymer layers, we used atomic force microscopy (AFM) in tapping mode and ambient conditions (MultiMode 8, Bruker). For phonon dynamics measurements, we used time-resolved fs pump-probe spectroscopy with a pulse duration of $60 \mathrm{fs}$ and a repetition rate of $250 \mathrm{kHz}$, from a Ti: $\mathrm{Al}_{2} \mathrm{O}_{3}$ laser at a wavelength of $800 \mathrm{~nm}$, already utilized in previous work [75]. Since PC has a glass transition temperature of about $140{ }^{\circ} \mathrm{C}$ [210], it was in the experiment important to avoid a too strong heating. Thus we have chosen an incident power of $150 \mathrm{~mW}$ with a Gaussian laser spot radius of $60 \mu \mathrm{m}$ (FWHM) leading to an optical fluence of about $5 \mathrm{~mJ} / \mathrm{cm}^{2}$. Results of this method were fitted to a numerical finite differences time domain (FDTD) model in order to get information about wave propagation inside the system [216-218].

\subsection{Results and discussion}

According to the acoustic mismatch model in 3D [86, 209], where phonon waves are treated following Fresnel's equations, the transmission probability for phonons from material 1 to 2 or vice versa is [219]:

$$
t_{1-2}\left(\theta_{1}\right)=t_{2-1}\left(\theta_{2}\right)=\frac{\frac{4 \rho_{2} v_{2}}{\rho_{1} v_{1}} \cdot \frac{\cos \theta_{2}}{\cos \theta_{1}}}{\left(\frac{\rho_{2} v_{2}}{\rho_{1} v_{1}}+\frac{\cos \theta_{2}}{\cos \theta_{1}}\right)^{2}}
$$

where $\rho_{1,2}$ are the corresponding densities of materials 1 or $2, v_{1,2}$ the group velocities of phonons in material 1 or 2 , and $\theta_{1,2}$ the incident phonon angles against 
the normal of the interface.

This transmission probability can be reduced by increasing the mismatch between the density and sound velocity of the two materials. For a great mismatch, on the one hand $\mathrm{W}$ has been chosen, which can be laser deposited with low surface roughness of only $0.3 \mathrm{~nm}$, as shown earlier [144,199]. On the other hand we chose PC as the corresponding counterpart in the multilayer. For polymers, the laser deposition process is more complex than for metals and can lead to drastic changes in polymer properties [220]. Therefore, single layers of PC have been deposited at different laser fluences (up to $250 \mathrm{~mJ} / \mathrm{cm}^{2}$ ) onto thin $\mathrm{SiN}$ substrates for NEXAFS studies at the K-absorption edge of carbon. In Figure $4.1 \mathrm{a}$, the NEXAFS measurements are depicted for several PC thin films. Independent of the laser fluence used during deposition, all curves show the absorption maxima typical for the different bondings in PC [221]. The small deviations from bulk PC concerning the intensity of the peaks are explainable by the process of incubation at the target [222].

In order to perform hardness measurements, several PC thin films with thicknesses of some $\mu \mathrm{m}$ (to minimize the influence of the substrate) were deposited with different laser fluences on Si substrates. Figure $4.1 \mathrm{~b}$ shows the corresponding curves of the Young's modulus $\mathrm{E}$ against the laser fluence indicating a drastic increase of hardness from $7.6 \mathrm{GPa}$ to $120 \mathrm{GPa}$. The strong increase of the elastic modulus can be related to the more and more intense laser radiation, which leads to increasing structural changes of the PC due to the laser energy of about $5 \mathrm{eV}$ used for deposition (corresponding to the wavelength of $248 \mathrm{~nm}$ ). At lowest laser fluence, the polymer properties are best preserved.

Since the acoustic mismatch between PC and W does not only depend on the difference in their corresponding densities, but also on the difference in sound velocities, a low value for $\mathrm{PC}$ in comparison to the high value for $\mathrm{W}$ is desirable. Here, one has to distinguish between two sound velocities, mainly the longitudinal $v_{L}=\sqrt{M / \rho}$ and transversal $v_{T}=\sqrt{G / \rho}$, that depend on the longitudinal modulus $M$ and the shear modulus $G$, respectively [223]. Since both moduli are linked to the E-modulus according to $M=\frac{E(1-v)}{(1+v)(1-2 v)}$ and $G=\frac{E}{2(1+v)}$, respectively, with the Poisson's ratio $v$, it is desirable to fabricate a soft polymer with a low E-modulus, which has the highest contrast to the hard $W$ and provides therefore the highest possible acoustic mismatch. As a consequence, a low laser fluence is necessary for the multilayer preparation.

Another important criterion for the use of a W/PC multilayer as a phononic crystal 

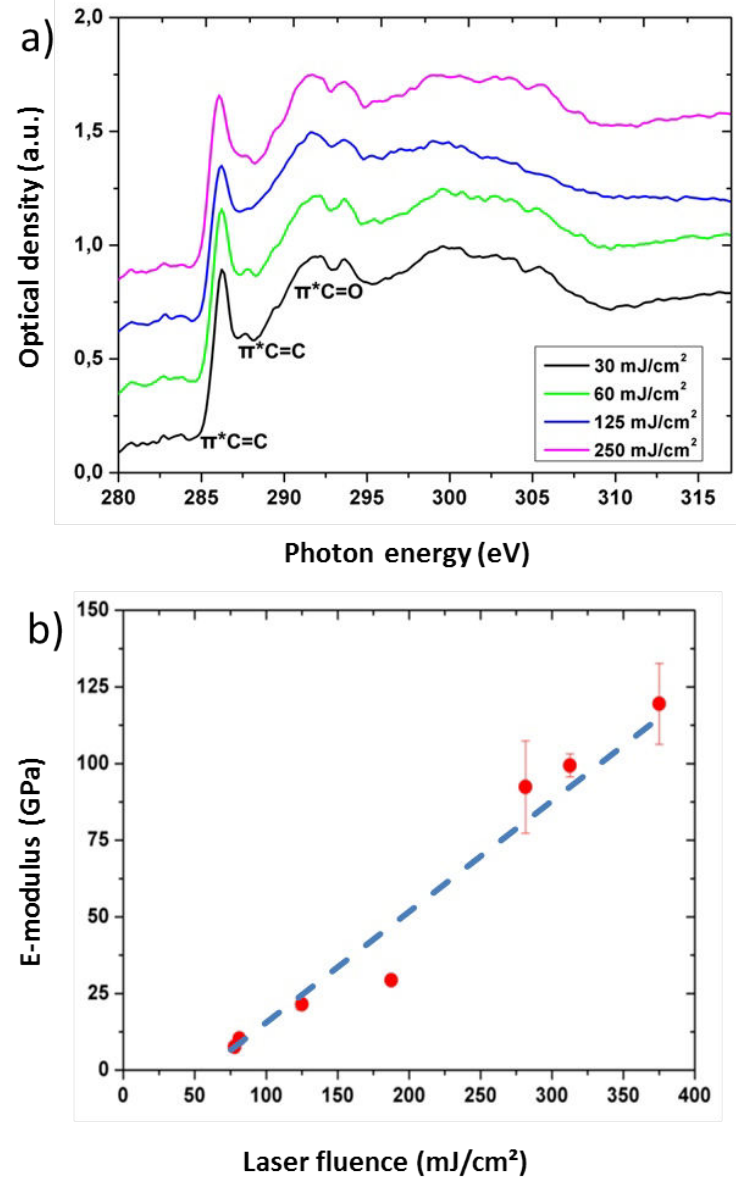

Figure 4.1.: NEXAFS measurements in a) show characteristic PC peaks as expected for a laser deposited polymer. Those remain stable at different laser fluences between $30 \mathrm{~mJ} / \mathrm{cm}^{2}$ and $250 \mathrm{~mJ} / \mathrm{cm}^{2}$. Hardness measurements in b) indicate a rising E-modulus of PC films with rising laser fluence because of increased structural change of the polymer (dashed line is guide to the eye).

is the roughness of the individual layers. As already mentioned above, $\mathrm{W}$ layers grown by PLD feature a RMS roughness of around $0.3 \mathrm{~nm}[197,199]$, but for PC layers, this was unknown so far. Again, a systematic analysis of the PC properties depending on laser fluence has been undertaken, this time concerning roughness. Figure $4.2 \mathrm{a}$ and $\mathrm{b}$ show exemplarily two AFM profiles of PC layers, one which has been deposited at $80 \mathrm{~mJ} / \mathrm{cm}^{2}$ and another one, where $310 \mathrm{~mJ} / \mathrm{cm}^{2}$ were used. It is clearly visible that a higher laser fluence results in a higher roughness, as also summarized in figure $4.2 \mathrm{c}$. Below $200 \mathrm{~mJ} / \mathrm{cm}^{2}$, a relatively low roughness of around $1 \mathrm{~nm}$ is observed, which rises up to a value of almost $10 \mathrm{~nm}$ at high laser fluences.

From all these results it can be concluded that a low laser fluence results in 
a)
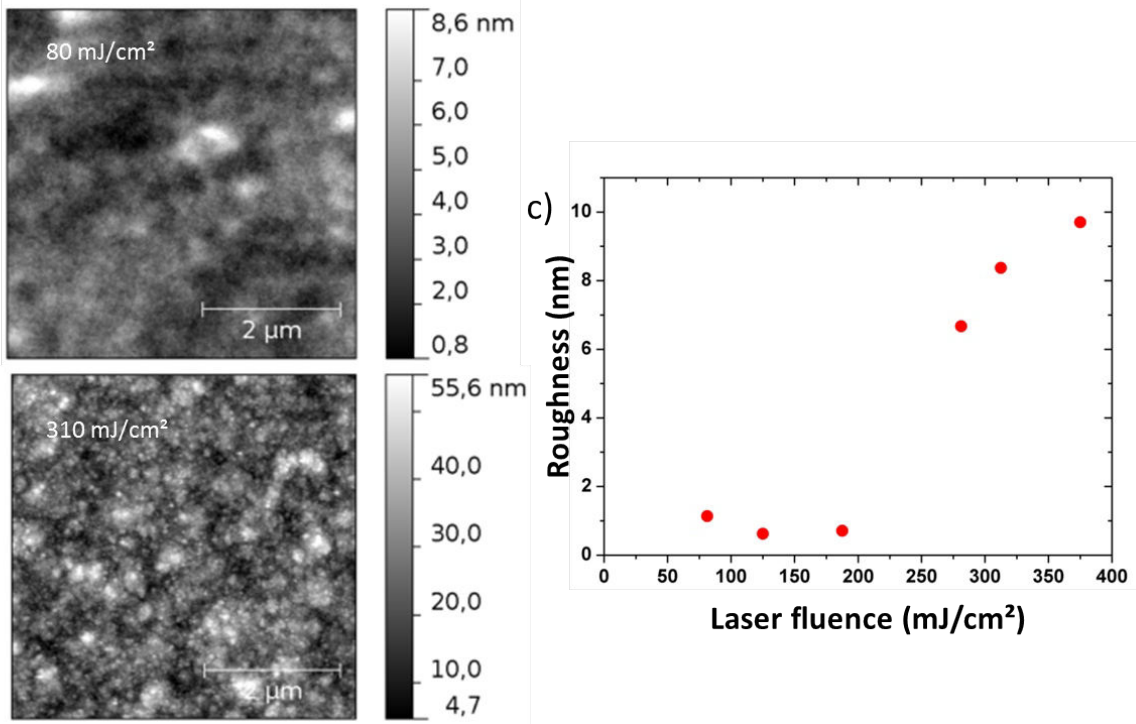

Figure 4.2.: Roughness investigations in PC films. a) and b) show AFM measurements on PC films deposited at laser fluences of $80 \mathrm{~mJ} / \mathrm{cm}^{2}$ and $310 \mathrm{~mJ} / \mathrm{cm}^{2}$, respectively. A corresponding graph in c) shows a significant roughness reduction towards $1 \mathrm{~nm}$ at laser fluences below $200 \mathrm{~mJ} / \mathrm{cm}^{2}$.

smoother layers and well defined interfaces, which is helpful when studying phonon propagation and reflection in W/PC multilayers. Thus, for the growth of the W/PC multilayers we chose laser fluences of $3.5 \mathrm{~J} / \mathrm{cm}^{2}$ for $\mathrm{W}$ and $80 \mathrm{~mJ} / \mathrm{cm}^{2}$ for PC.

Under these conditions, several W/PC multilayers have been laser deposited and analyzed by TEM and XRR. In figure $4.3 \mathrm{a}$ and $\mathrm{b}$, exemplarily TEM micrographs of a cross-section of such a structure are shown in different resolutions, which indicate the high quality of the W/PC multilayers. Due to the difference in particle mass and energy during deposition, PC grows very smoothly on top of W resulting in a low interface roughness, whereas the heavy $\mathrm{W}$ particles intermix slightly with the PC layer. This asymmetric intermixing process has already been shown elsewhere and originates from the occurrence of energetic ions during PLD [144].

$\mathrm{XRR}$ measurements performed on such multilayers support these results. In figure $4.3 \mathrm{c}$, the experimental curve of a multilayer with 8 periods of $\mathrm{W}$ and $\mathrm{PC}$ is depicted, which shows strong Bragg peaks even up to a scattering angle $2 \theta$ of 8 degrees already indicating sharp interfaces. From the fit of the simulated reflection curve to the experimental curve, the layer thicknesses of $W$ and PC of $8.3 \mathrm{~nm}$ and $10.5 \mathrm{~nm}$, respectively, and an interface roughness of $0.8 \mathrm{~nm}$ and $0.3 \mathrm{~nm}$ for $\mathrm{W}$ on PC and vice versa were determined.

Such a periodic multilayer with overall thickness of about $115 \mathrm{~nm}$ (schematically 

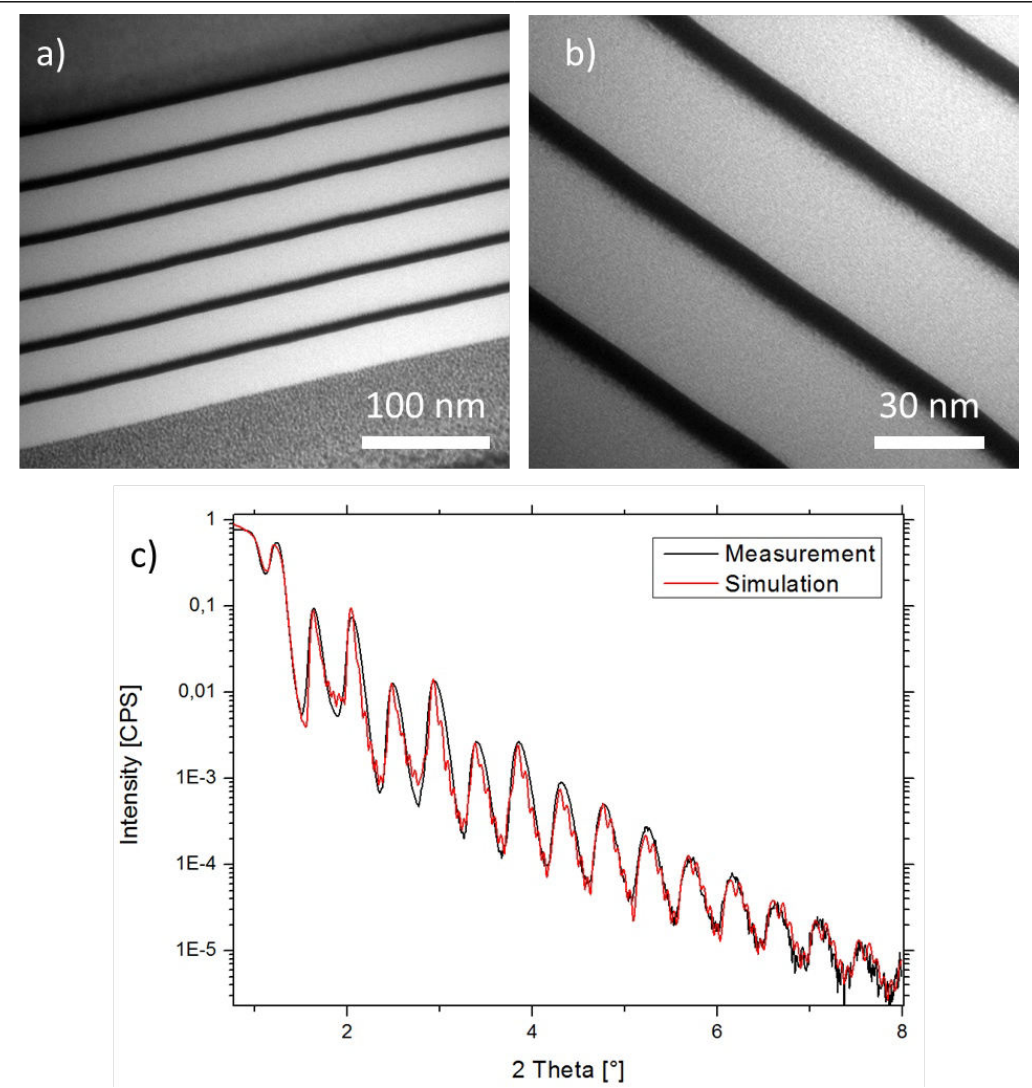

Figure 4.3.: a) and b) depict two TEM images in different magnification of a W/PC multilayer with layer thicknesses of $35 \mathrm{~nm}$ for PC (bright layers) and $7.5 \mathrm{~nm}$ for W (dark layers), respectively, showing a high quality thin film stack with smooth surfaces and a slight intermixing at the $\mathrm{W} / \mathrm{PC}$ interface. c) shows XRR measurements (black) and corresponding simulations (red) of a W/PC multilayer with single layer thicknesses around $10 \mathrm{~nm}$.

sketched in the inset of figure 4.4 a) has now been analyzed by fs pump-probe reflectivity measurements. As shown in figure $4.4 \mathrm{a}$, after the initial peak, the reflectivity $R$ decreases and shows a fast oscillation within the first 50 ps. In order to obtain the frequency of this oscillation, first the background caused by thermal diffusion was subtracted from the data and then the residual reflectivity data $R^{\prime}$ were Fourier transformed. The Fourier amplitude spectrum (see figure $4.4 \mathrm{~b})$ shows a peak at a frequency $f_{p}$ of $318 \mathrm{GHz}$, corresponding to the fast oscillation. The temporal decay of this mode is characterized by a linewidth $\Delta f_{p}$ of $39 \mathrm{GHz}$. To interpret these results, we performed numerical FDTD simulations of the experiments [224]. While the experiment is surface sensitive, this numerical approach allows looking inside the sample. Note that because the lateral size of the laser spots is much larger than the total thickness of our sample, only the z-direction, which is normal to the multilayer stack, is relevant. The numerical 

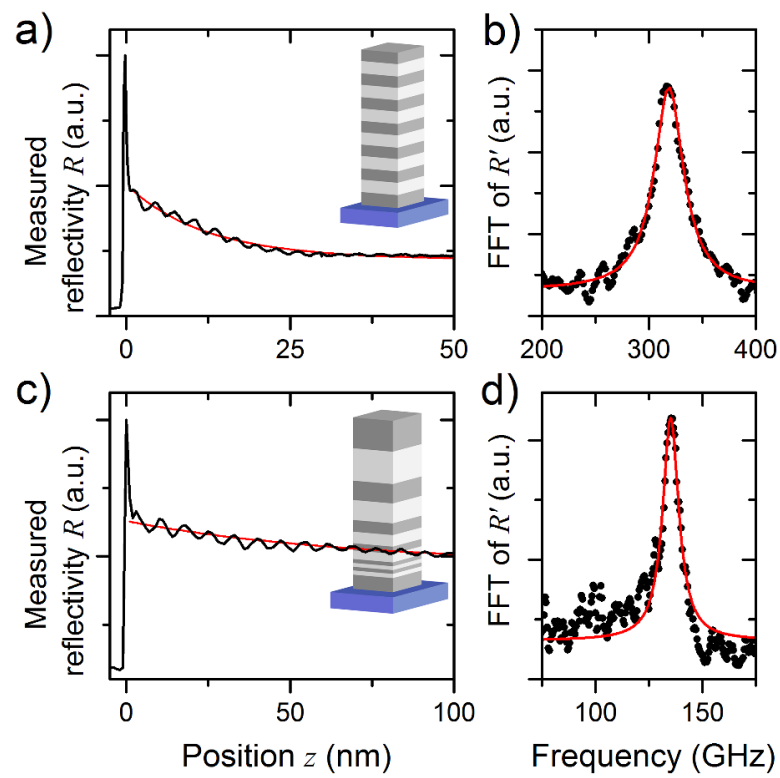

Figure 4.4.: Pump-probe reflectivity measurements, obtained from a) a periodic W/PC multilayer of 6 periods of $8.4 \mathrm{~nm} \mathrm{~W}$ (grey) and $9.1 \mathrm{~nm} \mathrm{PC} \mathrm{(white)} \mathrm{on} \mathrm{a} \mathrm{single} \mathrm{layer} \mathrm{of}$ $9.6 \mathrm{~nm} \mathrm{~W}$, deposited on a glass substrate (blue), and c) an aperiodic W/PC multilayer with layer thicknesses from $20.2 \mathrm{~nm} \mathrm{~W}$ and $21.8 \mathrm{~nm}$ PC down to $2.5 \mathrm{~nm} \mathrm{~W}$ and $2.7 \mathrm{~nm}$ PC according to a Fibonacci series again on a $9.6 \mathrm{~nm} \mathrm{~W}$ layer deposited on a glass substrate. In both datasets, an exponentially decaying background (in red) is superposed by a coherent oscillation. After removal of the background, the residual reflectivity $\mathrm{R}^{\prime}$ was Fourier transformed. b) and d) show Fourier amplitude spectra corresponding to the periodic and aperiodic sample. The frequency intervals selected in these plots contain the spectral peaks corresponding to the aforementioned coherent oscillations.

model implements laser light absorption, thermal diffusion and thermal expansion. The latter gives rise to elastic dynamics, which is described as well. We present our results in terms of maps of locally Fourier transformed strain $\tilde{\epsilon}(f, z)=$ $\operatorname{FFT}\{\epsilon(t, z)\}$. Note that the strain $\epsilon=-\partial u / \partial z$ related to a dynamic mode is not continuous at the interface between two dissimilar materials, in contrast to the stress $\sigma=c_{11} \epsilon$.

Adjusting the speed of sound of $W$ to $5260 \mathrm{~m} / \mathrm{s}$, and the value of PC to $2100 \mathrm{~m} / \mathrm{s}$, the numerical model qualitatively reproduces the experimental findings. Here, especially the adjusted speed of sound $v_{L}=2100 \mathrm{~m} / \mathrm{s}$ of PC is interesting, because from material parameters alone, one would expect $v_{L}=2600 \mathrm{~m} / \mathrm{s}$ for PC. The reduction in the longitudinal sound velocity can be attributed to an increased temperature, which is also known for other polymers [225]. The amount of velocity change implies an average temperature increase due to heat accumulation of $\Delta T=$ $50 \mathrm{~K}$ above room temperature RT during the experiment, although the time interval between successive laser pulses is $4 \mu \mathrm{s}$. For this, one has to take into account the 


\section{Confinement of phonon propagation in laser deposited Tungsten/Polycarbonate}

\section{multilayers}
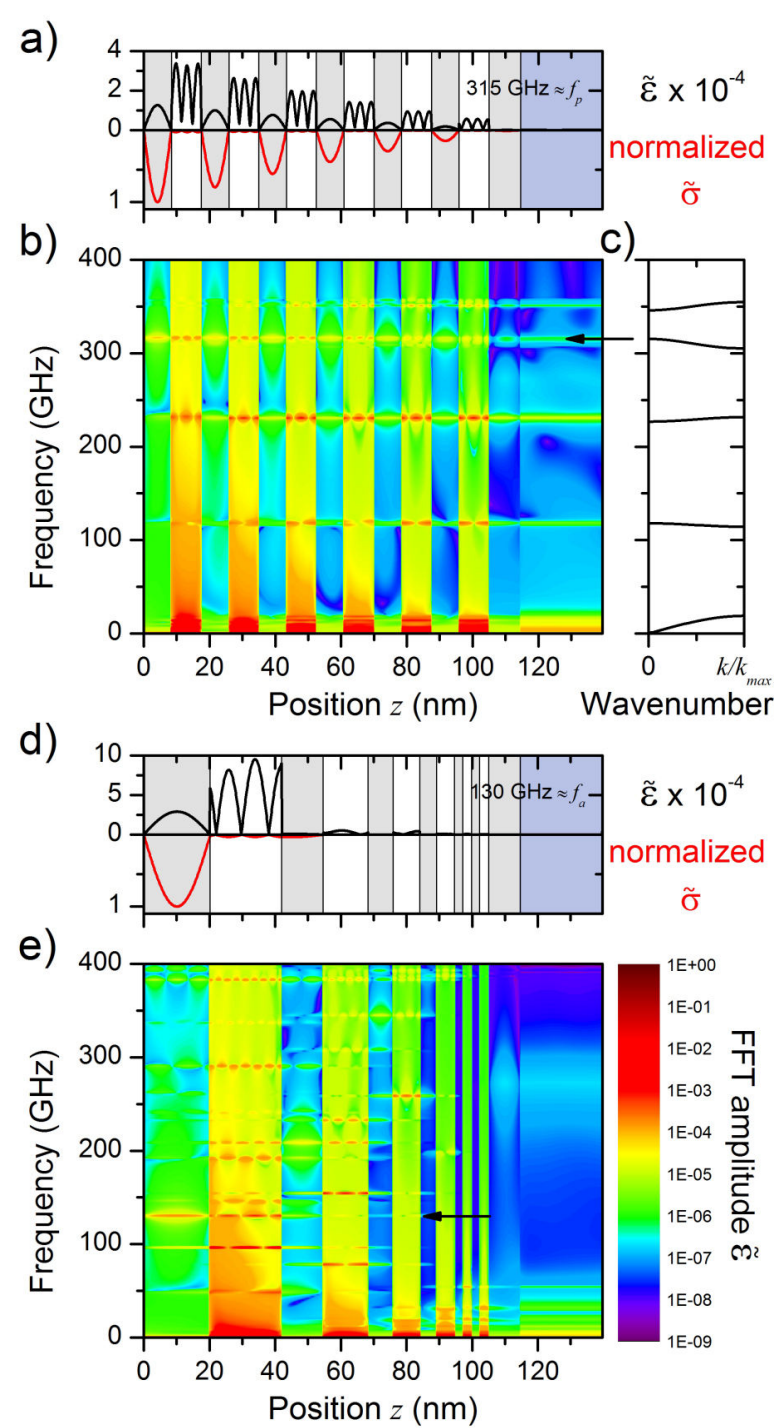

Figure 4.5.: Simulated pump-probe experiment. Results corresponding to the periodic sample are shown in a), b), and c), results corresponding to the aperiodic sample are shown in d) and e). b) and e) show maps $\tilde{\epsilon}(f, z)$ of locally Fourier transformed strain $\epsilon(t, z)$. The same logarithmic color scale was used for both plots. In each plot, an arrow marking the strongest experimentally observable mode at $315 \mathrm{GHz}$ for the periodic stack and at $135 \mathrm{GHz}$ for the aperiodic one is included. a) and b) show sections through these strain maps for specific frequencies, as well as the corresponding stress. $\mathrm{W}$ is shown in grey, while PC is displayed in white and the substrate in blue. c) shows a theoretical band structure calculation for the periodic sample.

strong temperature dependence of the speed of sound $\frac{\mathrm{d} v_{L}}{\mathrm{~d} T}(R T) \approx-10 \mathrm{~m} / \mathrm{sK}$ in PC [226]. This temperature increase of $50 \mathrm{~K}$ is very reasonable, because we found that a doubling of the incident laser fluence led to the destruction of the sample, because the glass temperature $T_{g}$ was reached [227]. In the case of $W, v_{L}=5260 \mathrm{~m} / \mathrm{s}$ is not far from literature values and the temperature dependence is neglibible [211]. 
In particular, in the numerical model we find for the periodic sample a mode at $315 \mathrm{GHz}$ with a linewidth of $1.9 \mathrm{GHz}$, marked by an arrow in figure $4.5 \mathrm{~b}$. The section through the strain map at $315 \mathrm{GHz}$ (see figure 4.5 a) highlights the collective character of the dynamics, which extends throughout almost the whole multilayer. The superlattice structure of such a multilayer accounts for phonon reflection at the interfaces and, taking into account coherent phonons with a mean free path larger than the superlattice spacing, even interference can occur [228-230]. This leads to significant changes in the dispersion relation and band gaps form that prevent several phonon modes from transmitting [231-233]. Figure $4.5 \mathrm{c}$ shows a calculation of this band structure using Rytov's equation [234]. In our periodic system with its artificially fabricated acoustic band structure the dominant mode at $315 \mathrm{GHz}$ can be seen as a Bloch-like eigenmode of the periodic multilayer. Such an eigenmode can coherently propagate though the superlattice only getting disturbed by scattering centers like rough interfaces or impurities, where phonons lose their phase information and transport becomes diffusive. Note that, while the simulation suggests the excitation of dynamics in all bands (as shown in figure $4.5 \mathrm{~b}$ ), the experimental observations only confirm dynamics in the first and fourth band. Dynamics in the second $(\sim 120 \mathrm{GHz})$ and third band $(225 \mathrm{GHz})$ cannot be seen experimentally. This apparent contradiction can be understood by the details of the measurement process, in which a signal contribution carrying information about elastic dynamics builds up by integrating a product of the local electric light field E and strain $\epsilon$ [235]:

$$
R \sim \int \mathrm{d} z \frac{\partial n}{\partial \epsilon} \epsilon E^{2}
$$

In this formula, $R$ is the measured reflectivity change and $\partial n / \partial \epsilon$ describes in a linear approximation the material dependent change of the refractive index when being strained. Looking at Figure $4.5 \mathrm{~b}$, one sees that the modes excited in the second and third band have strong strain amplitudes in the PC, and weak amplitudes in the W. In the PC in particular several nodal lines can be seen. Thus the spatial integration in Equation 4.2 effectively cancels out a contribution to the measured signal. This is the first reason for the missing appearance of this dynamics in the experimental data. Secondly, one should consider that together with the strength of the light field, the parameter $\partial n / \partial \epsilon$ determines the sensitivity on dynamics in the different layers. The light field decays exponentially in the metallic layers on a scale of $23 \mathrm{~nm}$. This implies that the method is especially 


\section{Confinement of phonon propagation in laser deposited Tungsten/Polycarbonate}

multilayers

surface sensitive. Note that $\partial n / \partial \epsilon$ is often not well-known. In total we conclude that the dominant appearance of dynamics in the fourth band not only reflects the larger amplitudes in the vicinity of the surface, but also indicates that the parameter $\partial n / \partial \epsilon$ is larger for $W$ than for PC. Let's finally consider now the dynamics in the first band. Here, frequencies are lowest, and thus the wavelengths are larger than the individual layer thicknesses. Especially no nodal line inside a PC layer appears. Therefore sensitivity to dynamics in the first band does not vanish. Note that in this article the focus lies on high frequency dynamics, associated with the fourth band. Regarding the first band, the quantitative agreement between experiment and simulation is not as good. We attribute this to transient changes of elastic properties in PC, which was not taken into account in the simulation. If the periodicity had been fully perfect, all modes excited by the laser would have been able to pass the multilayer from the surface to the bottom. In fact, the thickness of the last $\mathrm{W}$ layer of $9.6 \mathrm{~nm}$ is slightly larger than the thickness of the preceding $\mathrm{W}$ layers. This is enough to detune the local resonance, and to stop the mode at $315 \mathrm{GHz}$ from penetrating into the last $\mathrm{W}$ layer, as the section through the strain map shown in figure 4.5 a reveals. This detuning finally blocks the radiation into the substrate. In order to foster this blocking effect to the maximum, an aperiodic sample has been designed, resembling a Fibonacci series starting at the substrate after the first $\mathrm{W}(9.6 \mathrm{~nm})$ layer with a PC $(2.7 \mathrm{~nm}) / \mathrm{W}(2.5 \mathrm{~nm})$ bilayer and ending at the surface with PC $(21.8 \mathrm{~nm}) / \mathrm{W}(20.2 \mathrm{~nm})$ (shown in figure $4.4 \mathrm{c})$. Again, in the raw data (figure $4.4 \mathrm{c}$ ), a fast oscillation can be easily identified. The Fourier spectrum shown in figure 4.4 (d) exhibits a corresponding peak at a frequency of $f_{a}=135 \mathrm{GHz}$, whose linewidth is $\Delta f_{a}=9 \mathrm{GHz}$. But, in contrast to the periodic multilayer, the simulated strain map of this aperiodic sample shows (see figure 4.5 e) that at a given frequency the elastic dynamics is almost always confined to a few layers only, because the missing long range symmetry only allows for local resonances. For instance, the dynamics at $f_{a}=135 \mathrm{GHz}$ experimentally found in the second sample can be explained by the simulation as a resonance (frequency $129 \mathrm{GHz}$, line width $1.1 \mathrm{GHz}$ ) of the top-most $\mathrm{W}$ layer (see section in figure 4.5 d). Note that due to an optical penetration depth of $23 \mathrm{~nm}$, the laser beam is almost fully absorbed within the first $\mathrm{W}$ layer. In this thick layer, the resonances have a lower frequency than in the following thinner layers. Therefore, the thin layers effectively block the passage of elastic waves coming from the surface, and radiation into the substrate is suppressed in a wide frequency range, which is not only an important result for phononics, but is also discussed as a route to 
minimize thermal transport [164]. This finding is supported by a closer look at the experimentally determined line widths in the Fourier-regime (shown in figures $4.4 \mathrm{~b}$ and $4.4 \mathrm{~d}$ ). In contrast to the simulation, where perfectly flat interfaces were assumed, experimental interface roughnesses give rise to additional scattering and thus lead to a cumulative broadening of the line width with each passed interface. One finds in the periodic case a ratio $f_{p} / \Delta f_{p}$ of about 9.6, whereas the aperiodic sample features a higher value of $f_{a} / \Delta f_{a}$ of 15 . This increase by a factor of 1.5 can be interpreted as a fingerprint of a reduced accumulated interfacial scattering: In the aperiodic sample, the mode simply extends over less interfaces than in the periodic sample. Furthermore, the interfaces are also decisive for the thermal conductivity of the material. Using a simple heat conduction model one can cautiously estimate the thermal conductivity of the $\mathrm{W} / \mathrm{PC}$ multilayer. Using the incident laser power $P_{0}$ of $150 \mathrm{~mW}$ deposited within the Gaussian laser spot with radius $\mathrm{r}$ of $60 \mu \mathrm{m}$ on the multilayer surface and the measured temperature increase of about $\Delta T=50 \mathrm{~K}$, the cross-plane thermal conductivity given by

$$
\kappa \approx \frac{P_{0} d_{t o t}}{\pi r^{2} \Delta T}
$$

Where $d_{t o t}$ is the total thickness of the multilayer, should be in the order of about $0.03 \mathrm{~W} / \mathrm{mK}$. This estimation is indeed reasonable: in a multilayer one can estimate the thermal conductivity by

$$
\kappa=\frac{d_{W}+d_{P C}}{\frac{d_{W}}{\kappa_{W}}+\frac{d_{P C}}{\kappa_{P C}}+n R_{b d}}
$$

where $d_{W}$ and $d_{P C}$ are the layer thicknesses of the $\mathrm{W}$ and PC layers, respectively, $n$ is the number of interfaces and $R_{b d}$ is the interfacial thermal resistance [148]. With the thermal conductivities of $W=167 \mathrm{~W} / \mathrm{mK}$ and $W=0.2 \mathrm{~W} / \mathrm{mK}[210,211]$, and a typical interfacial thermal resistance $R_{b d} \approx 10^{-7} \mathrm{~m}^{2} \mathrm{~K} / \mathrm{W}$ for thin films $[60,86,236]$, a value for the thermal conductivity of $0.08 \mathrm{~W} / \mathrm{mK}$ is evaluated, which is in the same order of magnitude. Both values indeed indicate that the thermal conductivity of the W/PC multilayer should be very low. 


\subsection{Conclusion}

In this work we have shown that nanoscale $\mathrm{W} / \mathrm{PC}$ multilayers are appropriate candidates for acoustic layered materials due to the great difference in material properties of the adjoining components. In order to fabricate such structures with high quality, the PLD process had to be investigated in detail, especially concerning the deposition of the polymer. At low laser fluences, PC thin films show all characteristic peaks in the NEXAFS spectra, lowest hardness and minimum layer roughness. In the $\mathrm{W} / \mathrm{PC}$ multilayers, the $\mathrm{PC} / \mathrm{W}$ interfaces are sharp (PC on W), while slight intermixing occurs at the other interface, as seen in the XRR and TEM measurements. The fs pump-probe reflectivity measurements on periodic W/PC structures show strong phonon mode confinement and phonon localization, restricting the propagation of coherent phonons according to the band structure defined by the periodicity of the multilayer. In order to improve this phonon mode confinement, aperiodic multilayers have been fabricated, where the aperiodic structure filters even more modes especially from the coherent regime and thus further reduces phonon propagation. In summary, the nanoscale thin film synthesis of such different components as $\mathrm{W}$ and PC in multilayers is proved to be challenging, but lead to an interesting new material combination. Such structures are promising for future applications as two-dimensional phononic crystals and might be also a promising approach as a very-low thermal conductivity material.

\section{Acknowledgment}

We would like to thank Volker Radisch for his support at the focused ion beam. This work was supported by the Deutsche Forschungsgemeinschaft (SFB 1073: "Atomic scale control of energy conversion" Project A02 and A03). 


\section{Thermal conductivity measurement by transient thermoreflectometry using high-fluence excimer laser pulses at $248 \mathrm{~nm}$ wavelength}

Florian Döring ${ }^{1}$, and Hans-Ulrich Krebs ${ }^{1}$

1 Institut für Materialphysik, Georg-August-University Göttingen, FriedrichHund-Platz 1, 37077 Göttingen, Germany

High Temperatures-High Pressures

Accepted for publication on 11 August 2016

\subsection{Abstract}

In many modern materials, thermal properties and especially the thermal conductivity become more and more important. Thus, a wide research field evolved around measuring thermal conductivities. Here, we show an implementation of a versatile transient thermoreflectometry technique for measuring thermal conductivity that utilizes a pulsed $\mathrm{KrF}$ laser with $248 \mathrm{~nm}$ wavelength and $20 \mathrm{~ns}$ pulse duration for pumping energy into the surface. Time resolved reflectivity changes from a continuous wave laser at $643 \mathrm{~nm}$ are measured using a fast Si-photodiode and an oscilloscope. To optimize this setup, thermoreflectivity properties were studied for different metal transducers as well as optical thicknesses. Since $20 \mathrm{~ns}$ are a comparatively long time-scale, the measurements could be fitted to an approximated analytical solution of 


\section{Thermal conductivity measurement by transient thermoreflectometry using}

high-fluence excimer laser pulses at $248 \mathrm{~nm}$ wavelength

the heat equation for thermal properties. In this way we could show that the thermal conductivities of different materials can be determined with in most cases sufficiently high accuracy by this relatively simple and flexible technique.

Keywords: Transient thermoreflectometry, thermal conductivity, pulsed laser deposition, thermoreflectivity, thermoreflectance, heat transfer

\subsection{Introduction}

In many fields of modern physics thermal properties become more and more important [4]. Especially the thermal conductivity is a characteristic that plays an increasingly important role for instance in industrial electronics, energy science, thermoelectrics and phononics $[35,81]$. In order to measure thermal conductivities for different materials, there are several possibilities like the 3-omega technique, laser flash methods, pyrometry, photodisplacement or thermoreflectance methods [63,64,66,73,167].

Among all those measurement techniques, the transient thermoreflectance (TTR) method is prominent, because it provides a non-contact, non-destructive way to measure thermal conductivity with low sample preparation effort. TTR belongs to the class of optical pump-probe measurement schemes, where a short laser pulse (in the fs or ns regime) is used to excite the surface of a sample. The absorbed energy from the pump pulse leads to an increase of temperature and since the refractive index is temperature dependent, the reflectivity of the sample changes until equilibrium is reached again [181]. The reflectivity dynamic at the surface after the pump pulse is accessed either by a time-delayed probe pulse or by a second, continuous-wave laser. With a sufficient temporal resolution, one can deduce information about several transport processes (such as thermal diffusion, acoustic investigations, electron-phonon-interactions, thermal imaging, and thermal boundary resistance measurements) from the surface reflectivity change by comparing the measured data to a theoretical model, which can be done either analytically or numerically $[72,85,176,193,237,238]$.

In a typical TTR-setup a Ti:Sapphire laser with ultrashort pulses, comparatively weak pulse energies and very high repetition rates is used. In contrast, we chose a KrF-laser for our measurements, which is normally used for pulsed laser deposition (PLD) of thin films [51,239]. This allows us to work with much 
higher laser energies and, thus, with long equilibration times between pulses. Furthermore, the laser pulse length is comparatively long with $20 \mathrm{~ns}$, meaning that acoustic dynamics, stress pulses, electron-phonon interactions and other fast processes are decayed long before the pulse ends and the measurement of the reflectivity dynamics starts, allowing to focus on slower processes like thermal diffusion.

Aim of this work is to show that a new kind of TTR setup that uses a KrF laser with a wavelength of $248 \mathrm{~nm}$ for surface heating and a continuous wave laser at $643 \mathrm{~nm}$ for probing is especially well suited to measure the thermal conductivity of materials with sufficient precision. In order to obtain the thermal properties from the measured data, we will present a comprehensible approximated analytical solution of the heat conduction equation that was used as a fit function for the measurements. Finally, first experiments on different materials are presented and compared with literature values, and a quasi-material approach for an extension of this method on thin film multilayer thermal conductivity measurements is suggested.

\subsection{Materials and Methods}

For pumping energy into the sample, a $\mathrm{KrF}$ laser with wavelength of $248 \mathrm{~nm}$, pulse duration of $20 \mathrm{~ns}$ and fluences in the range of $50 \mathrm{~mJ} / \mathrm{cm}^{2}$, which is much lower than the ablation threshold for metals, was used. For probing the reflectivity change at the surface, a stable continuous wave laser at $643 \mathrm{~nm}$ wavelength with low noise was applied. Both laser beams have been guided and focused by typical optical elements in order to obtain a well-working setup with sufficient local overlap of both beams. The reflectivity signal is measured by a fast Si-photodiode and analyzed within an oscilloscope. The whole measurement scheme is sketched in Figure 5.1.

In order to deposit the metal transducer layer on top of the samples, the method of pulsed laser deposition (PLD) was applied, where the same $\mathrm{KrF}$ laser as for TTR was used [51,199]. At this, the pulses are guided into an ultra-high-vacuum chamber and are focused on the targets chosen for deposition. As promising transducer materials, thin films of $\mathrm{Al}, \mathrm{W}, \mathrm{Cr}, \mathrm{Ti}$ and $\mathrm{Cu}$ were fabricated from commercially available bulk targets. 


\section{Thermal conductivity measurement by transient thermoreflectometry using} high-fluence excimer laser pulses at $248 \mathrm{~nm}$ wavelength

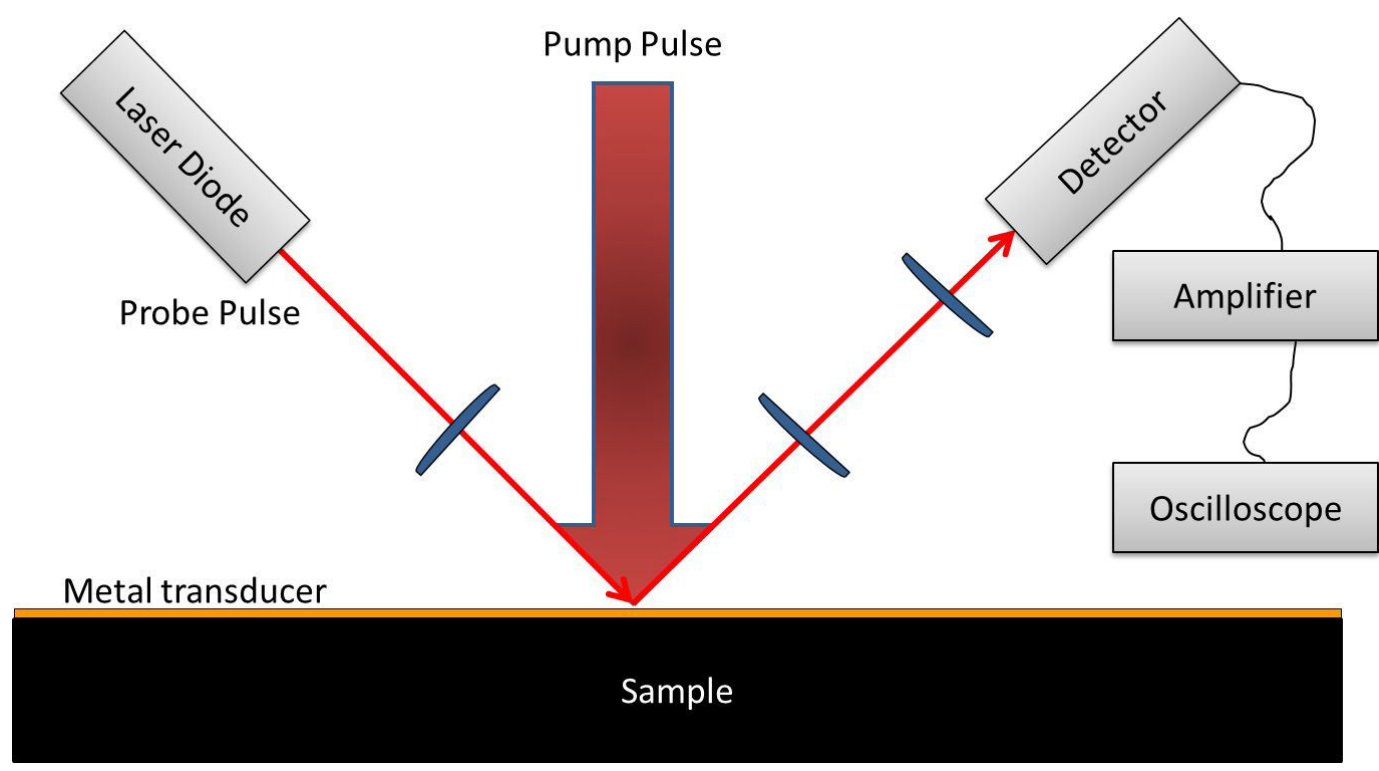

Figure 5.1.: Sketch of the transient thermoreflectometry measuring setup. A pump laser pulse with $248 \mathrm{~nm}$ wavelength is focused onto a metal transducer on top of the sample. At this point, the reflectivity of a continuous laser with $643 \mathrm{~nm}$ wavelength is measured with a fast Si-photodiode. The timeresolved change in reflectivity after a pump pulse is evaluated with a fast oscilloscope.

\subsection{Results and discussion}

The method of TTR is based upon the temperature-dependent change in reflectivity at the surface of a sample. The change in reflectivity and the change in temperature are coupled according to the following formula [180]:

$$
\frac{\Delta R}{R}=\frac{1}{R} \frac{\partial R}{\partial T} \Delta T=C \Delta T
$$

$\Delta R$ is the change in reflectivity, $R$ the initial reflectivity, $C$ the thermoreflectivity coefficient and $\Delta T$ the change in temperature. While the thermoreflectivity coefficient is mainly temperature-independent [240,241], it changes largely for different metals and laser wavelengths. For a wavelength of $643 \mathrm{~nm}$, as used for our reflected probe beam, $\mathrm{Al}, \mathrm{Ti}$ and $\mathrm{Cu}$ have a comparably high thermoreflectivity coefficient with an absolute value of around $8.5 \cdot 10^{-5} \mathrm{~K}^{-1}$ according to the literature [242]. A high thermoreflectivity coefficient is of great importance for the 
signal to noise ratio of the measurement setup, since it scales the reflectivity change proportionally to the temperature change. Moreover, also a good reflectance for our probe beam, as well as a good absorbance of our $248 \mathrm{~nm}$ pump beam is very important.

Because a $\mathrm{KrF}$ laser has never been used for such a measurement method before, we first had to figure out, which material and which layer thickness are most suitable as transducer top-layer in order to get an as high as possible thermoreflectivity signal. In order to clarify this, $50 \mathrm{~nm}$ of $\mathrm{Al}, \mathrm{W}, \mathrm{Cr}, \mathrm{Ti}$ and $\mathrm{Cu}$ were deposited each on a $100 \mathrm{~nm}$ thick $\mathrm{ZrO}_{2}$ layer on a Si-substrate. The $\mathrm{ZrO}_{2}$ layer has been chosen as a thermal blocking layer in order to strengthen the overall signal and to make the different curves better comparable. Then, each sample was excited by the same laser fluence and the time-dependent reflectivity change was measured (see in Figure 5.2). One can clearly see that all five metals have significantly different thermoreflection properties.

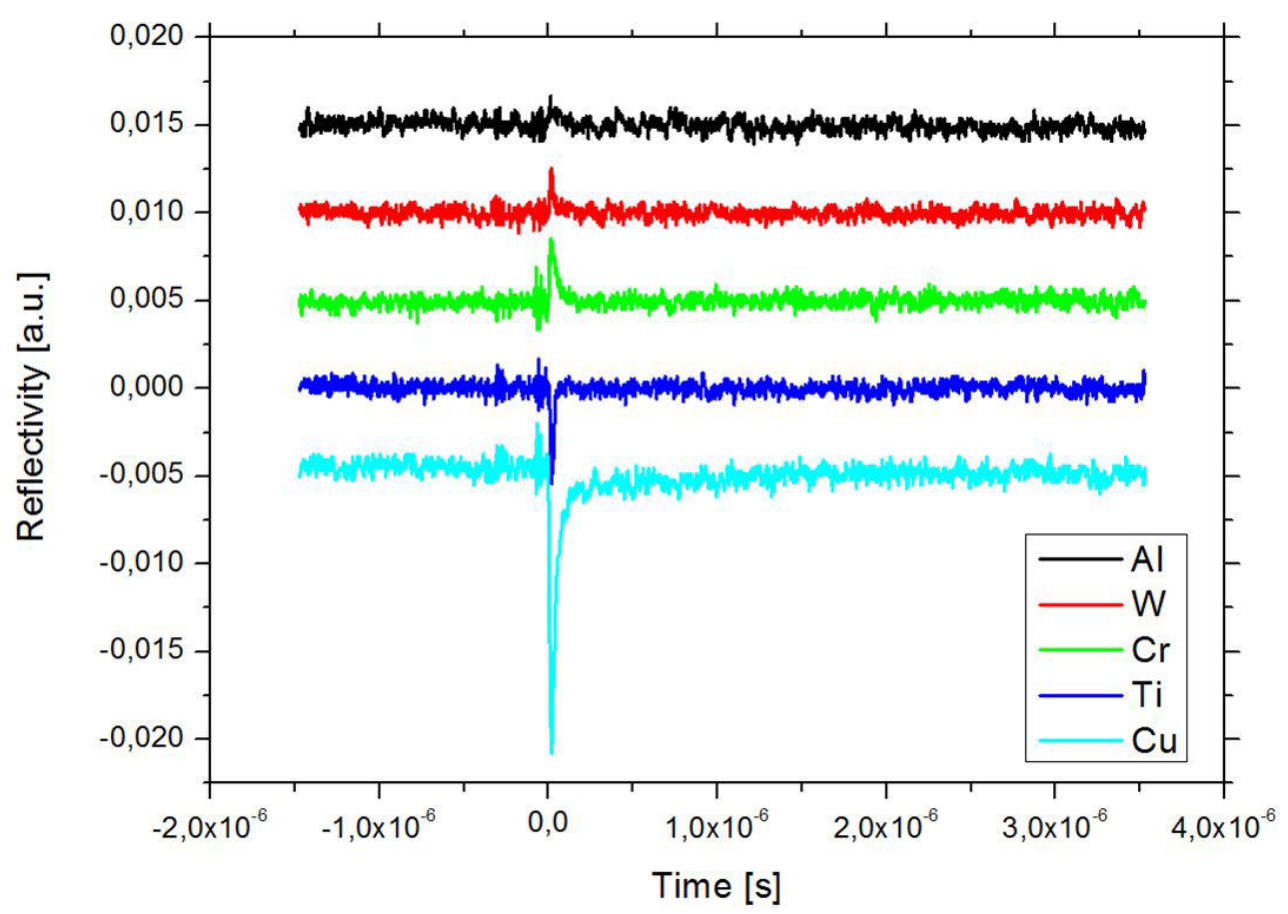

Figure 5.2.: Time-dependent reflectivity signal for various metal transducers. Despite $\mathrm{Al}$, $\mathrm{Ti}$ and $\mathrm{Cu}$ have almost the same thermoreflectance coefficients, the measured reflectivity change is greatest for $\mathrm{Cu}$ due to the highest values in absorption at $248 \mathrm{~nm}$ and reflectivity at $643 \mathrm{~nm}$.

While $\mathrm{Al}, \mathrm{W}$ and $\mathrm{Cr}$ show a positive change in thermoreflectance, the same effect is negative for $\mathrm{Ti}$ and $\mathrm{Cu}$. Although, according to literature, $\mathrm{Al}$, $\mathrm{Ti}$ and $\mathrm{Cu}$ have a 


\section{Thermal conductivity measurement by transient thermoreflectometry using}

high-fluence excimer laser pulses at $248 \mathrm{~nm}$ wavelength

comparably high thermoreflectance coefficient [242], we could show that the final change in reflectance is highest in $\mathrm{Cu}$ and lowest in Al. Reason for this is that the change in reflectance (at $643 \mathrm{~nm}$ ) is not only proportional to the thermoreflectivity coefficient, but also to the change in temperature and the overall reflectivity at $248 \mathrm{~nm}$. Since $\mathrm{Al}$ has a very high reflectivity at $248 \mathrm{~nm}$, which hinders the laser energy absorption of the pump pulse, the change in temperature at the surface and with it the reflectivity signal is comparatively small. Also for Ti, the reflectivity at $248 \mathrm{~nm}$ is higher than for $\mathrm{Cu}$, while at the same time the reflectivity at $643 \mathrm{~nm}$ is lower than for $\mathrm{Cu}$. All in all, $\mathrm{Cu}$ was proved to be the material of choice with highest signal for efficient TTR measurements.

In order to find the optimal thickness of the $\mathrm{Cu}$ transducer layer, $\mathrm{Cu}$ films with different layer thicknesses were pulsed laser deposited onto $\mathrm{Si}$ substrates that were coated with $100 \mathrm{~nm} \mathrm{ZrO}_{2}$ before. The results of the reflectivity measurements are shown in Figure 5.3, where it is clearly visible that at a layer thickness of around $50 \mathrm{~nm}$ the maximum signal arises. This is reasonable due to the fact that with smaller layer thickness absorption and reflection of the incident beam is not fully guaranteed, whereas with larger thickness, heat losses in the top layer become more important, which as a consequence reduce $\Delta T$ and thereby the measurable reflectivity change.

For the deposition of the $50 \mathrm{~nm} \mathrm{Cu}$ layers on top of the samples, the optimal PLD conditions concerning a maximized deposition rate with a minimized droplet density had to be investigated. With a laser fluence of $2.9 \mathrm{~J} / \mathrm{cm}^{2}$, a high deposition rate of $0.0065 \mathrm{~nm} /$ pulse (at a target-to-substrate distance of $50 \mathrm{~mm}$ ) and a low droplet density was achieved. Moreover, the adhesion of the $\mathrm{Cu}$ films on different kinds of substrates is very strong, as it is generally the case for pulsed laser deposited metals because of the impinging energetic particles, sub-surface growth and resputtering of surface contaminations [51]. Thus, thin film adhesion is strong enough that the measurements are reproducible and no cracks or dissolutions of the top-most $\mathrm{Cu}$ film occur.

For testing the TTR method and measuring the thermal conductivities, different bulk materials $\left(\mathrm{Al}_{2} \mathrm{O}_{3}, \mathrm{MgO}, \mathrm{SrTiO}_{3}, \mathrm{ZrO}_{2}\right.$ and glass) were taken and coated with $50 \mathrm{~nm} \mathrm{Cu}$ by PLD. Afterwards, they were studied in the TTR setup, where their time-dependent reflectivity changes after the pump pulse were determined. The results of these measurements are shown in Figure 5.4.

After the initial time with a constant reflectivity, the pump pulse hits the $\mathrm{Cu}$ layer at time $0 \mathrm{~s}$ and heats it up for $20 \mathrm{~ns}$. At this, the amount of temperature change 

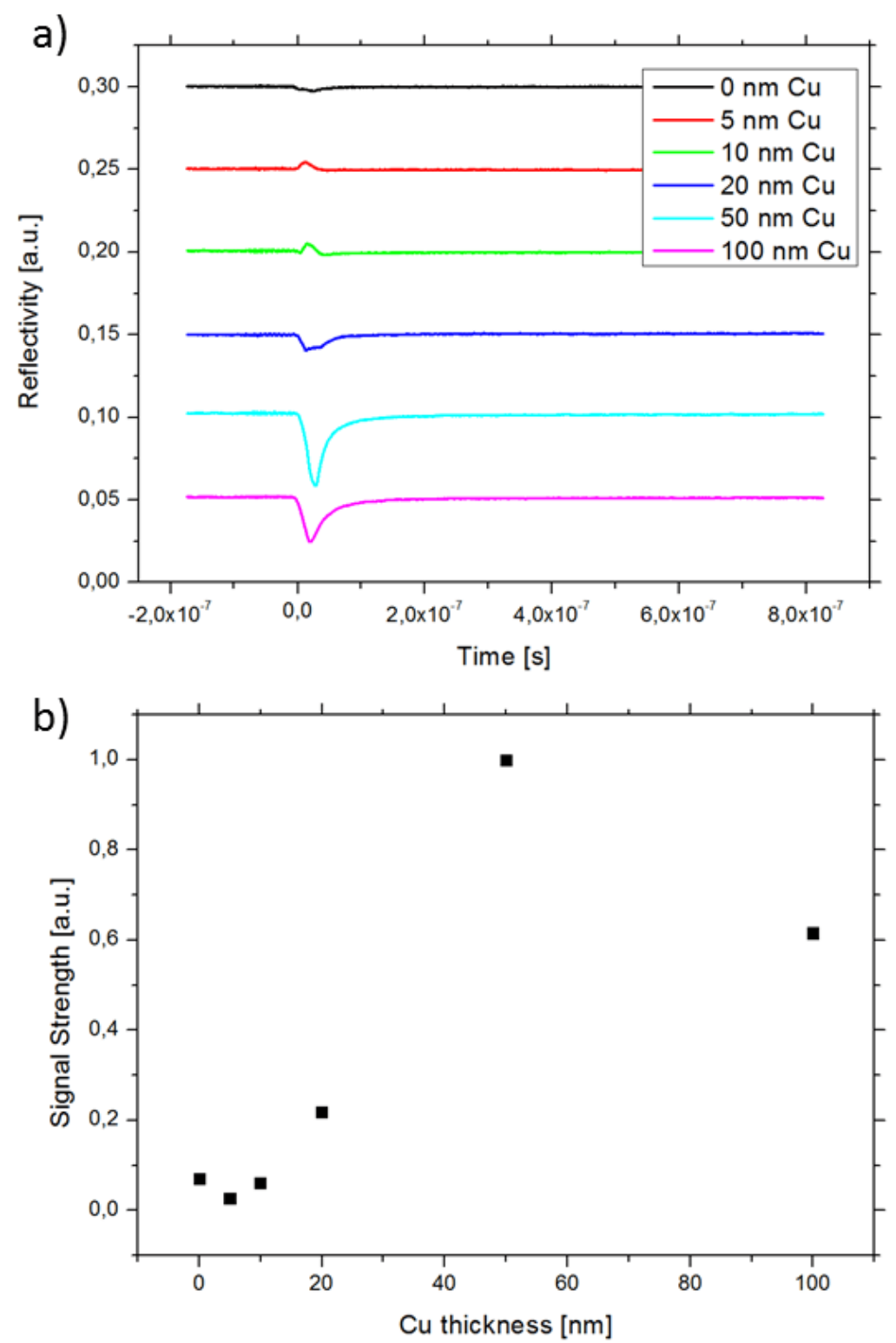

Figure 5.3.: a) Time-resolved reflectivity signal for several $\mathrm{Cu}$ toplayer thicknesses. b) Signal strength is greatest for transducer layers around $50 \mathrm{~nm}$, because of maximized pump power absorbtion and probe laser reflection while heat flow losses in the transducer layer are still small.

$\Delta T$ depends on the thermal conductivity $\mathcal{K}$, heat capacity $c$ and density $\rho$ of the investigated material. Those three thermophysical properties can be combined in the value of the thermal effusivity $e=\sqrt{\kappa c \rho}$. The higher this value $e$, the lower is the change in temperature at the surface and vice versa. Therefore, for different materials, which have different thermophysical properties, we obtain quite different curve progressions (see Figure 5.4). 


\section{Thermal conductivity measurement by transient thermoreflectometry using} high-fluence excimer laser pulses at $248 \mathrm{~nm}$ wavelength

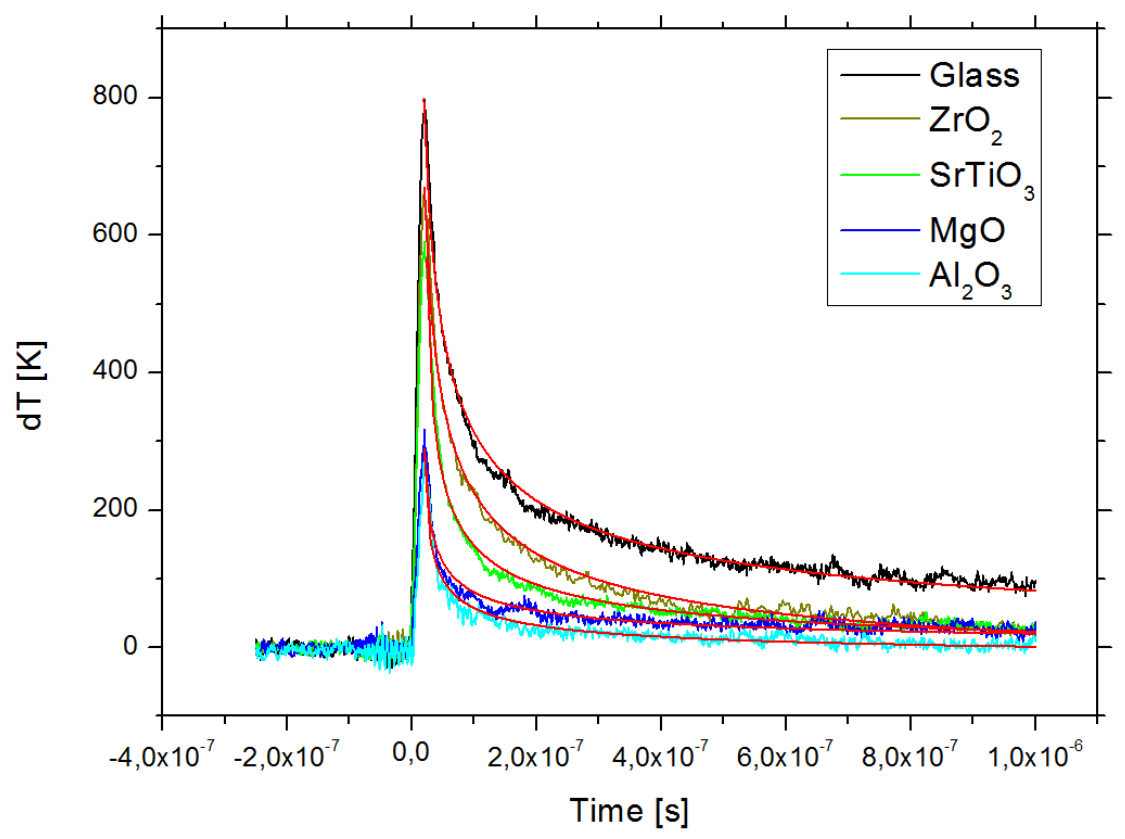

Figure 5.4.: Different oxidic materials were measured with the TTR method. In order to get information about thermal properties, the measurement data sets have been fitted to an approximated analytical solution of the heat conduction equation (plotted in red).

In order to extract the value of the thermal conductivity of the materials out of the measurements, one has to solve the heat conduction equation in one dimension [74]:

$$
\frac{\partial T}{\partial t}=D \nabla^{2} T
$$

Here, $D=\frac{\kappa}{c \rho}$ is the thermal diffusivity and the approximation of a one-dimensional heat transfer is reasonable due to the difference in pump and probe spot sizes. Equation 5.2 can be solved for instance by Laplace or Fourier transformation and yields the time- and space-dependent temperature field after absorption of a Dirac heat pulse of strength $Q$ at the surface of an infinite medium in the following way [74]:

$$
T(z, t)=\frac{Q}{e \sqrt{\pi t}} \exp \left(\frac{-z^{2}}{4 D t}\right)
$$


where $t$ is the time after the pump pulse, $z$ the distance below the surface and $T(0,0)=0$. Since we can only get information about the temperature at the surface, we have to consider $z=0$, which simplifies equation 5.3 in the form:

$$
T(z=0, t)=\frac{Q}{e \sqrt{\pi t}}
$$

According to this formula, the temperature at the surface would decay proportional to $1 / \sqrt{t}$, which is not a bad approximation to describe the experimental data, but only truly correct for infinite sample thicknesses, instantaneous excitations without a penetration depth and no other losses.

In order to account for the $\tau=20 \mathrm{~ns}$ broad laser pulse, which is assumed to be of a square-wave shape, Duhamel's theorem [184] can be applied leading to a convolution of the time distribution $\theta(t)$ of the laser pulse with the solution $T(z=0, t)$ for a Dirac pulse [183]:

$$
T_{D}(z=0, t)=\int_{0}^{t} \theta\left(t^{\prime}\right) \cdot T\left(z=0, t-t^{\prime}\right) d t^{\prime}=\frac{2 Q}{\tau e \sqrt{\pi}}(\sqrt{t}-\sqrt{t-\tau})
$$

Equation 5.5 can now be used as a fit function for the experimental values measured by TTR. The fitting curves for the here-mentioned data sets are also included in Figure 5.4 leading at first to a value for the thermal effusivity $e$. From this, the thermal conductivity $\kappa=\frac{e^{2}}{\rho c}$ can be determined by using literature values for the density $\rho$ and the heat capacity $c$ of the corresponding material. The hereby determined thermal conductivity values are shown in Table 5.1 in comparison to literature values.

Table 5.1.: Comparison of thermal conductivities between literature and experimental values obtained by the TTR-method [243-247].

\begin{tabular}{lcc}
\hline Material & Literature $\kappa[\mathrm{W} / \mathrm{mK}]$ & Measured $\kappa[\mathrm{W} / \mathrm{mK}]$ \\
\hline Glass & $1.2-1.4(300 \mathrm{~K}-1100 \mathrm{~K})$ & $1.5 \pm 0.17$ \\
$\mathrm{ZrO}_{2}$ & $1.7-2(300 \mathrm{~K}-1300 \mathrm{~K})$ & $1.9 \pm 0.2$ \\
$\mathrm{SrTiO}_{3}$ & $15-10(200 \mathrm{~K}-400 \mathrm{~K})$ & $11.6 \pm 1.3$ \\
$\mathrm{MgO}$ & $24-30(400 \mathrm{~K}-50 \mathrm{~K})$ & $32.0 \pm 4.0$ \\
$\mathrm{Al}_{2} \mathrm{O}_{3}$ & $46(300 \mathrm{~K})$ & $42.5 \pm 4.1$ \\
\hline
\end{tabular}

Doubtless, some incertitudes arise for instance from laser power fluctuations (which can be reduced by averaging over a couple of experiments), inaccuracies of the laser fluence measurements as well as the $\mathrm{Cu}$ layer thickness, usage of literature 


\section{Thermal conductivity measurement by transient thermoreflectometry using}

high-fluence excimer laser pulses at $248 \mathrm{~nm}$ wavelength

values for the density and heat capacity, approximations of the underlying theory, such as heat losses, sample thickness effects and an approximated laser pulse shape. Moreover, the substrate temperature is increased above room temperature at least for a short time during and after the laser pulse, where a temperaturedependent thermal conductivity should be applied. Thus, the expected uncertainty depends on several effects and can be estimated to be around $10 \%$. But nevertheless, it becomes apparent from Table 1 that all the experimental values determined by our TTR measurements lie relatively close to the literature data, although a relatively simple experimental set-up and analysis were performed.

One should note that this TTR technique can also be applied for thermal conductivity measurement of different kinds of multilayer films. Since the pumpprobe scheme excites and measures only at the surface and integrates due to the long pulse length over the underlying material, one can, in the case of multilayers consisting of two materials with individual layer thicknesses $d_{1}$ and $d_{2}$ and total thickness $d_{t o t}$, come up with a 'quasi-material' approach, where the contribution of a bilayer is seen as one structural unit. Then, this quasi-material is described by an averaged density $\rho_{\text {avg }}=\rho_{1} \frac{d_{1}}{d_{\text {tot }}}+\rho_{2} \frac{d_{2}}{d_{\text {tot }}}$, heat capacity $c_{\text {avg }}=\frac{\rho_{1} \frac{d_{1}}{t_{\text {tot }}} c_{1}+\rho_{2} \frac{d_{2}}{\text { tot }_{2}} c_{2}}{\rho_{1} \frac{d_{1}}{d_{\text {tot }}}+\rho_{2} \frac{d_{2}}{\frac{t_{\text {tot }}}{t_{\text {tot }}}}}$ and thermal conductivity of $\kappa_{a v g}=\frac{d_{t o t}}{\frac{d_{1}}{\kappa_{1}}+\frac{d_{2}}{\kappa_{2}}+\frac{n}{G_{i}}}$ that depends on the conductivities of both materials 1 and 2, as well as on the interface thermal conductance $G_{i}$ and number of interfaces $n$ [148]. First TTR experiments on multilayer structures consisting of oxides with metal blocking layers have already shown that this model can successfully be applied and a significant decrease in cross-plane thermal conductivity of the multilayers compared to the pure oxides occurs due to the contribution of the interface resistance (to be published elsewhere).

\subsection{Conclusion}

In summary we have shown that the TTR experiment using the combination of an UV laser at $248 \mathrm{~nm}$ for heating and a $643 \mathrm{~nm}$ laser for probing is a comparatively handy method for measuring thermal conductivities with a low sample preparation effort and high measurement throughput capabilities. Our approach featuring comparatively long laser pulses in the UV regime, allows neglecting shorter timescale processes, such as electron-phonon interaction and phonon dynamics, which take place on fs and ps timescales, while concentrating 
on the much slower thermal processes and thus allowing the determination of thermal conductivities in different materials with in most cases sufficiently high accuracy.

\section{Acknowledgment}

The authors acknowledge financial support by the "Deutsche Forschungsgemeinschaft" (SFB 1073 in project A02). 



\section{Minimized thermal conductivity in highly stable thermal barrier $\mathrm{W} / \mathrm{ZrO}_{2}$ multilayers}

Florian Döring ${ }^{1}$, Anna Major ${ }^{1}$, Christian Eberl ${ }^{1}$, Hans-Ulrich Krebs ${ }^{1}$

1 Institut für Materialphysik, University of Göttingen, Friedrich-Hund-Platz 1, 37077 Göttingen, Germany

Applied Physics A 122:872, pp 1-5 (2016)

doi:10.1007/s00339-016-0405-0

Accepted for publication on 29 August 2016

Published online 06 September 2016 as Rapid Communication

\subsection{Abstract}

Nanoscale thin film multilayer materials are of great research interest since their large number of interfaces can strongly hinder phonon propagation and lead to a minimized thermal conductivity. When such materials provide a sufficiently small thermal conductivity and feature in addition also a high thermal stability they would be possible candidates for high temperature applications such as thermal barrier coatings. For this article, we have used pulsed laser deposition in order to fabricate thin multilayers out of the thermal barrier material $\mathrm{ZrO}_{2}$ in combination with $\mathrm{W}$, which has both a high melting point and high density. Layer thicknesses were designed such that bulk thermal conductivity is governed by the low value of $\mathrm{ZrO}_{2}$, while ultrathin $\mathrm{W}$ blocking layers provide a high number of interfaces. By this phonon scattering, reflection and shortening of mean free path lead to a 
significant reduction in overall thermal conductivity even below the already low value of $\mathrm{ZrO}_{2}$. In addition to this, x-ray reflectivity measurements were conducted showing strong Bragg peaks even after annealing such multilayers at $1300 \mathrm{~K}$. Those results identify $\mathrm{W} / \mathrm{ZrO}_{2}$ multilayers as desired thermally stable, low conductivity materials.

Keywords: Pulsed laser deposition, thermal conductivity, thermal stability, transient thermoreflectometry, nanoscale heat transport, thin film multilayer

\subsection{Introduction}

Thin film multilayers feature outstanding material properties for applications either in the field of optics, electronics or phononics [4,248]. Especially the thermal conductivity, which is an important parameter for a lot of possible applications, has been studied in detail in different nanostructures [81]. In thin multilayer films the thermal transport can be strongly tailored by interfaces due to scattering, reflection and mean free path shortening of phonons at interfaces $[75,91]$. As described by the acoustic- and diffuse mismatch model, transition of phonons from one material into another can be strongly prohibited by the difference of the densities of phononic states at each side of an interface $[59,86,209]$. By modern thin film methods one can fabricate multilayers of different materials such as metals, oxides, semiconductors or even polymers that feature a very high interface density, leading to a tremendous decrease of thermal conductivity [39]. Particularly, pulsed laser deposition (PLD) allows the fabrication of such multilayers consisting of different material classes $[47,48]$. By combining hard and heavy metals such as $\mathrm{W}$ together with oxidic ceramics, one can prepare thin films, where thermal conductivity from hot electrons is prohibited by insulating layers while phononic thermal conducitivity gets suppressed by the dissimilar material properties at the interfaces. In the simplest picture derived from the acoustic mismatch theory, the probability of energy transmission $\alpha_{1 \rightarrow 2}$ from material 1 with its density $\rho_{1}$, sound velocity $v_{1}$ and therefore its acoustic impedance $Z_{1}=\rho_{1} v_{1}$ to material 2 with its impedance $Z_{2}=\rho_{2} v_{2}$ is given by [86]:

$$
\alpha_{1 \rightarrow 2}=\frac{4 Z_{1} Z_{2}}{\left(Z_{1}+Z_{2}\right)^{2}}
$$


In the $\mathrm{W} / \mathrm{ZrO}_{2}$ system, the high density of $\mathrm{W}$ provides for the difference in acoustic impedances across the interfaces, leading to a reduced phonon transmission probability and therefore to a reduced thermal conductivity in a multilayer system. For the oxidic part of the multilayer, we chose the thermal barrier material $\mathrm{ZrO}_{2}$ since it has a low thermal conductivity and is thermally very stable with a melting point above $2700^{\circ} \mathrm{C}$ [249]. In combination with $\mathrm{W}$ with its even higher melting point of above $3400^{\circ} \mathrm{C}$, we expect a thermally stable multilayer with a strongly decreased thermal conductivity [39,250-252]. The aim of this paper is to prepare such thin film multilayers consisting of $\mathrm{W}$ and $\mathrm{ZrO}_{2}$ by PLD and to investigate them in terms of thermal conductivity and thermal stability. For thermal conductivity measurements a transient thermoreflectometry (TTR) technique is used in order to measure samples with different interface densities. For thermal stability measurements, multilayers of $\mathrm{W}$ and $\mathrm{ZrO}_{2}$ were annealed at different temperatures and afterwards characterized by $x$-ray reflectivtiy (XRR) measurements, where the occurrence of strong Bragg peaks indicates an intact multilayer structure.

\subsection{Experimental}

$\mathrm{W} / \mathrm{ZrO}_{2}$ multilayers were pulsed laser deposited from commercially available bulk targets onto Si substrates using a typical KrF-Laser $(248 \mathrm{~nm}$ wavelength, $10 \mathrm{~Hz}$ repetition rate, $20 \mathrm{~ns}$ pulse duration) in a standard setup [51,199]. The growth temperature in the ultrahigh vacuum chamber $\left(10^{-8} \mathrm{mbar}\right)$ was around $300 \mathrm{~K}$ and the target-to-substrate distance was chosen to be $7 \mathrm{~cm}$. Layer thicknesses were measured by in-situ rate measurements with an oscillating quartz crystal (Inficon SQM 160) and ex-situ via XRR measurements (Bruker D8 Discover with $\mathrm{Cu}-\mathrm{K} \alpha$-radiation) also yielding interface roughnesses from fitting simulated XRR curves on experimental ones using LEPTOS 7 (Bruker). Transmission electron microscopy images were taken using a FEI Titan and thermal conductivity data were measured by a TTR method, where the time-dependent change in reflectivity after laser pulse heating is measured and fitted with an analytical solution of the one-dimensional heat equation. For annealing we used a vacuum oven (KnürrHeinzinger) combined with a thermocouple (Eurotherm). 


\subsection{Results and discussion}

For fabricating high quality multilayers of $\mathrm{W}$ and $\mathrm{ZrO}_{2}$, deposition conditions had to be used, where the deposition rates are sufficiently high and droplet densities on the substrate surface are low. For $W$, a laser fluence of around $3 \mathrm{~J} / \mathrm{cm}^{2}$ leads to a deposition rate of $4 \mathrm{~nm} / 1000$ pulses, while the droplet density remains low, since the laser fluence is close to the deposition threshold. In the case of $\mathrm{ZrO}_{2}, \mathrm{a}$ laser fluence of $2.2 \mathrm{~J} / \mathrm{cm}^{2}$ was chosen, which is much higher than the deposition threshold. This results in deposition rates of around $34 \mathrm{~nm} / 1000$ pulses and almost no droplets at all, since formation of cones at the target is heavily suppressed by the high laser fluence. Under these conditions, $\mathrm{W} / \mathrm{ZrO}_{2}$ multilayers with different single layer thicknesses were grown with smooth layers as can be seen exemplarily in the high-resolution transmission electron micrograph of Fig. 6.1. Here, it should be noticed that the $\mathrm{ZrO}_{2}$ layers are amorphous after deposition. In order to obtain

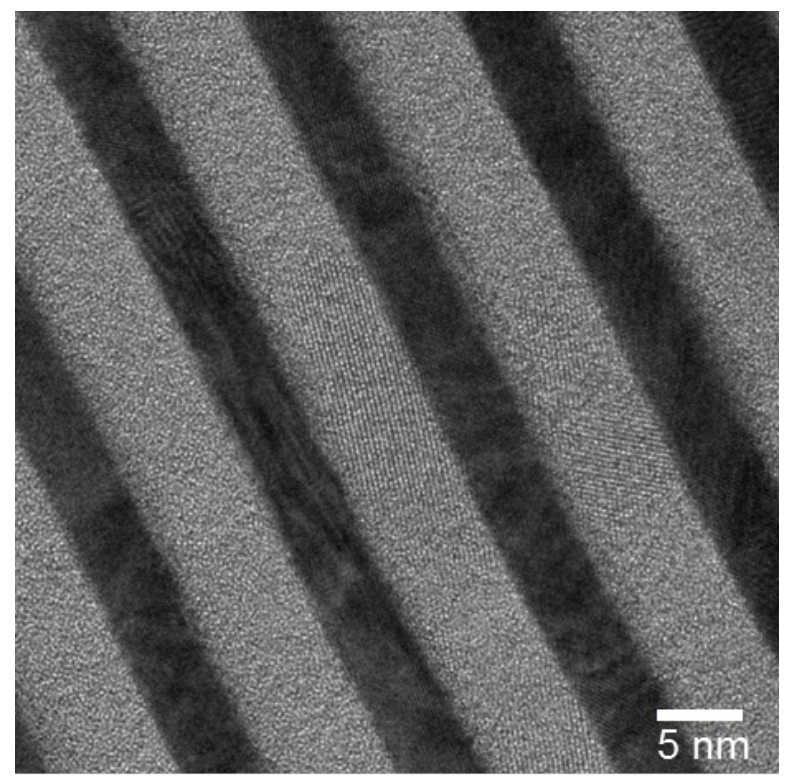

Figure 6.1.: High-resolution transmission electron micrograph of a $\mathrm{W} / \mathrm{ZrO}_{2}$ thin-film multilayer fabricated by pulsed laser deposition. The darker layers consist of $\mathrm{W}$ and the brighter ones of $\mathrm{ZrO}_{2}$

information about the structure and thermal stability of the $\mathrm{W} / \mathrm{ZrO}_{2}$ multilayers, they were measured by XRR and analyzed using the LEPTOS 7 program. At this, the periodicity of the layers can be shown by Bragg-peaks, while Kiessigfringes in the spectrum are an indication of a smooth surface. Typically, the interface roughness was determined in the range of $0.3-0.5 \mathrm{~nm}$. Afterwards, the multilayers were annealed for 1 hour at higher temperatures and analyzed again. 
This approach was proceeded until the maximum temperature of the oven was reached at around $1300 \mathrm{~K}$. Selected XRR measurements of a multilayer sample with $6 \mathrm{~nm}$ individual layer thickness after annealing at different temperatures are depicted in Fig. 6.2. Apart from a crystallization effect of $\mathrm{ZrO}_{2}$, the multilayer structure remains unchanged and keeps its layered structure even up to $1300 \mathrm{~K}$, which represents a very high thermal stability in comparison to other thin film systems $[253,254]$. For investigating the thermal conductivity in such multilayers,

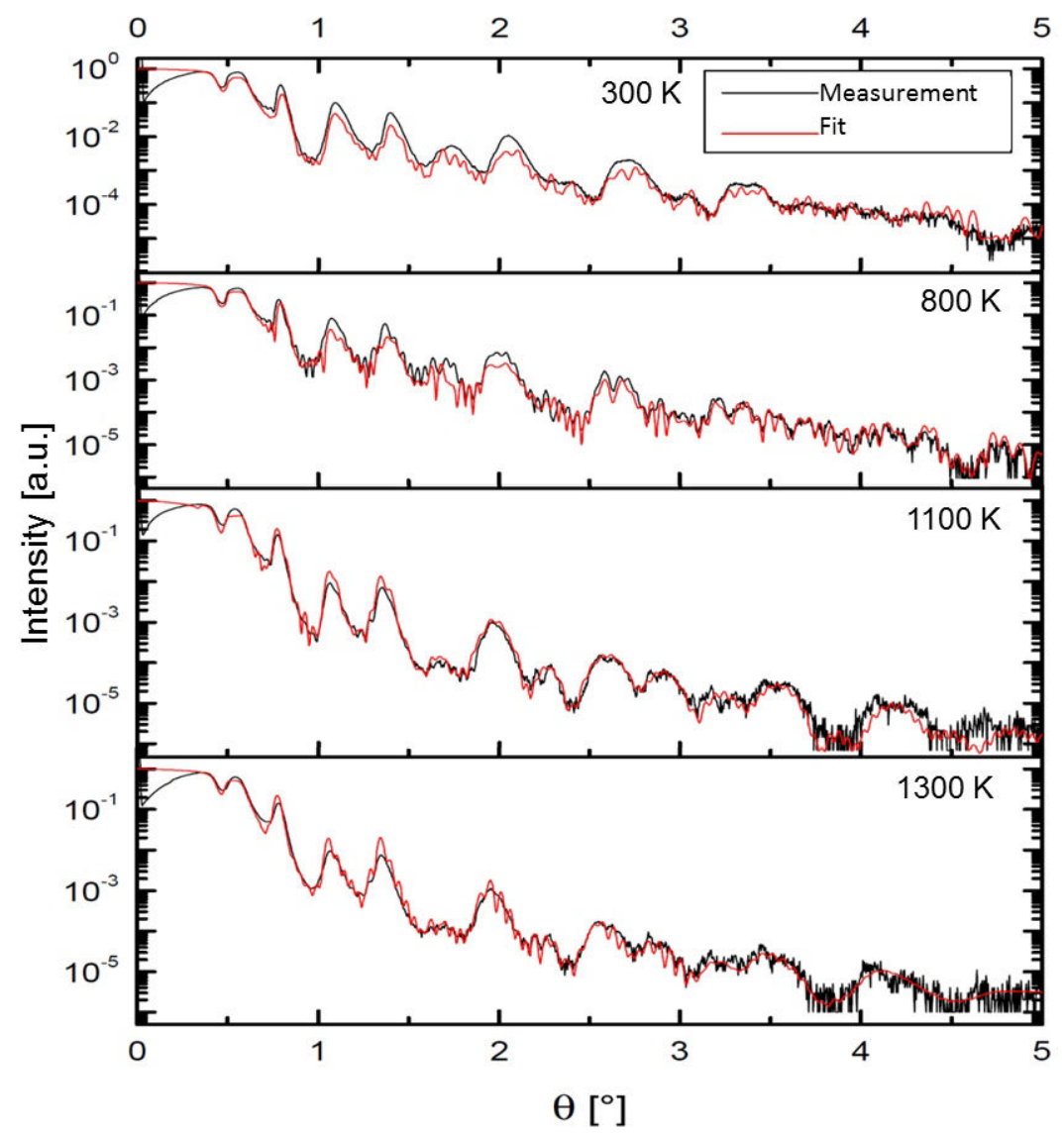

Figure 6.2.: $\mathrm{X}$-ray reflectivity measurements of a $\mathrm{W} / \mathrm{ZrO}_{2}$ multilayer with individual layer thicknesses of about $6 \mathrm{~nm}$. The structure at $300 \mathrm{~K}$ shows clear Bragg-peaks due to its layered structure. This multilayer structure is retained even after annealing up to $1300 \mathrm{~K}$. The fitting has been done with the program LEPTOS 7 using a simplex fitting algorithm.

different samples were pulsed laser deposited with overall thicknesses between 800 and $1000 \mathrm{~nm}$. For comparison, a $1 \mu \mathrm{m}$ thick $\mathrm{ZrO}_{2}$ film was fabricated, since this material is known to have an especially low thermal conductivity of around $1.9 \mathrm{~W} / \mathrm{mK}$ and is widely used for thermal barrier applications [244, 255]. For a further reduction of the thermal conductivity, thin $\mathrm{W}$ blocking layers were integrated in the $\mathrm{ZrO}_{2}$ structure resulting in a multilayer with a high number of 
periods $N=85$ with single layer thicknesses $d_{\mathrm{ZrO}_{2}}=8.6 \mathrm{~nm}$ and $d_{W}=1.6 \mathrm{~nm}$ for $\mathrm{ZrO}_{2}$ and $\mathrm{W}$, respectively. Thus, in this multilayer with total thickness $D$ of about $870 \mathrm{~nm}, 170$ interfaces were employed. In order to increase the interface density n even more, a multilayer with $N=170$ periods of $4.4 \mathrm{~nm} \mathrm{ZrO}_{2}$ and $0.6 \mathrm{~nm}$ thin $\mathrm{W}$ blocking layers was fabricated that exhibits 340 interfaces inside its $850 \mathrm{~nm}$ thickness. For comparing these layered $\mathrm{W} / \mathrm{ZrO}_{2}$ structures with an intermixed $\mathrm{W}$ $\mathrm{ZrO}_{2}$ alloy structure, another sample was fabricated, where the number of laser pulses on each target was so low that the resulting film became totally intermixed due to the energetic particles occurring during PLD [144,145]. The thickness of this sample was about $900 \mathrm{~nm}$ and consisted almost equally of $\mathrm{W}$ and $\mathrm{ZrO}_{2}$ in terms of nominal thicknesses. All samples show a greater overall thickness than the thermal penetration depth of the laser in this experiment, thus potential thickness effects on the thermal conductivity can only be attributed to the thicknesses of the individual layers and not to the sample thickness. XRR measurements on the multilayers as well as on the alloy sample are shown in Fig. 6.3 together with corresponding simulations, which give information about layer thicknesses and interface roughnesses. It can be clearly seen that the multilayers exhibit sharp Bragg peaks due to the layered structure. From the high number of Bragg peaks and their relative sharpness it can be concluded that the layer thicknesses are pretty uniform, but never identical within the multilayers. In contrast, for the homogeneous alloy no Bragg peaks and thus no interfaces exist. All these samples have been coated with a $50 \mathrm{~nm}$ thick $\mathrm{Cu}$-layer that serves as a transducer layer for the optical pump-probe scheme utilized for TTR measurements. For this method, the $\mathrm{KrF}$ laser is used in order to pump laser energy into the surface and hence heating it up within its $\tau=20 \mathrm{~ns}$ pulse time. The reflectivity of the surface layer changes with temperature according to the Fresnel formulas, which is expressed in the thermoreflectivity coefficient of the $\mathrm{Cu}$ top layer [241]. $\mathrm{Cu}$ is the material of choice for this application since it has a high absorption at the wavelength of the pump beam $(248 \mathrm{~nm})$, a sufficiently high reflection $R$ at the wavelength of the probe beam $(643 \mathrm{~nm})$ and a high thermoreflectivity coefficient (with an absolute value of $C \approx 8.5 \cdot 10^{-5} \mathrm{~K}^{-1}$ ) [242], that allows to obtain a measurable reflectivity change $\Delta R$ after laser heating (temperature increase $\Delta T$ ) according to the first order approximation [178]:

$$
\frac{\Delta R}{R}=\frac{1}{R} \frac{\partial R}{\partial T} \Delta T=C \Delta T
$$




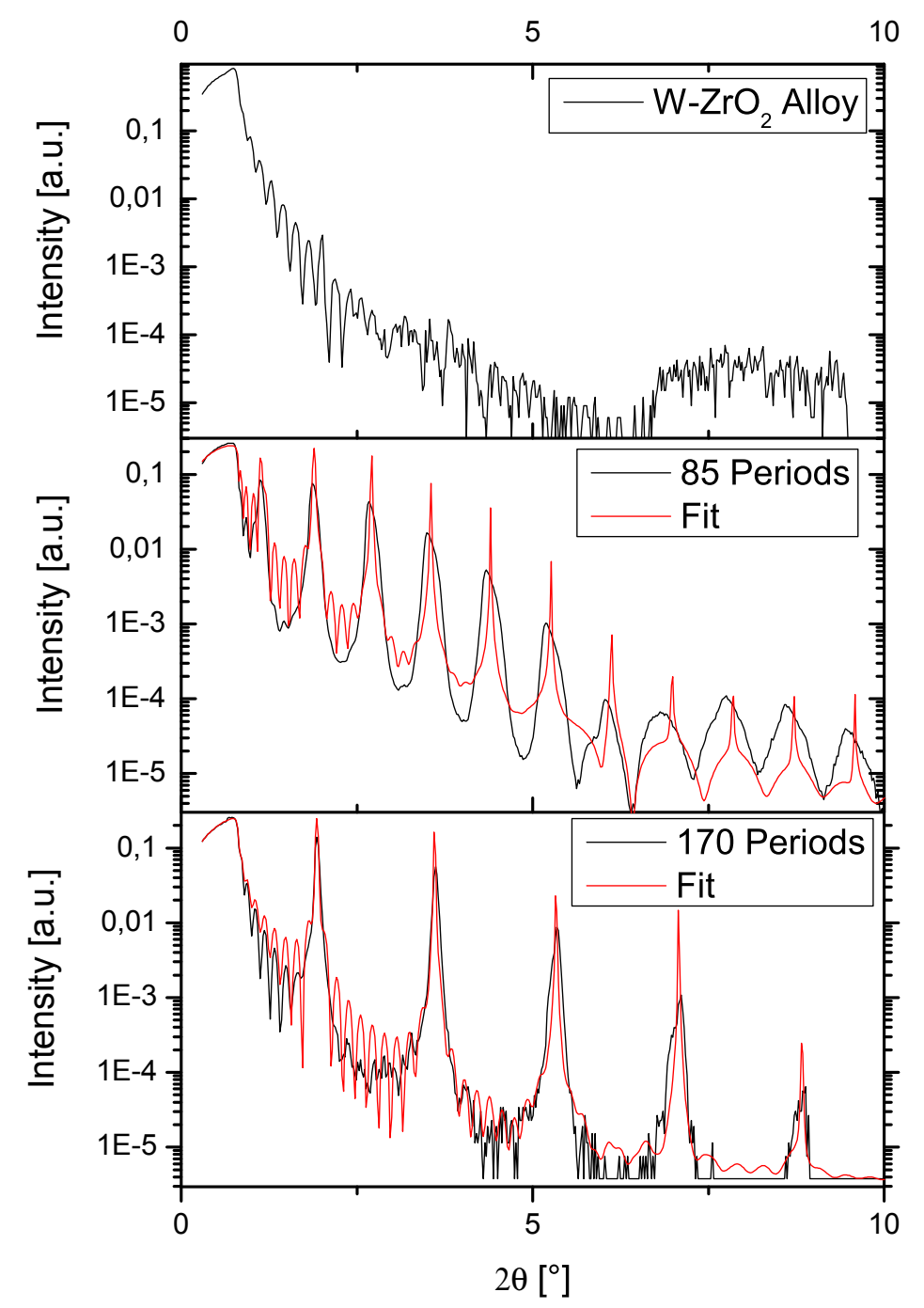

Figure 6.3.: X-ray reflectivity measurements of different $\mathrm{W} / \mathrm{ZrO}_{2}$ compounds. The first one shows an intermixed $\mathrm{W}-\mathrm{ZrO}_{2}$ alloy (proved by the absence of Bragg peaks) with almost identical nominal thicknesses of $\mathrm{W}$ and $\mathrm{ZrO}_{2}$. The second one shows a multilayer structure of 85 periods with $8.6 \mathrm{~nm} \mathrm{ZrO}_{2}$ and $1.6 \mathrm{~nm} \mathrm{~W}$, while the last one depicts a multilayer with 170 periods of $4.4 \mathrm{~nm} \mathrm{ZrO}_{2}$ and $0.6 \mathrm{~nm} \mathrm{~W}$. Fitting has been done with the program LEPTOS 7 using a simplex fitting algorithm.

After the pump pulse with strength $Q$ that is approximated by a square wave shape, the surface temperature decreases with time, depending on the thermal 
conductivity $\kappa$, density $\rho$ and heat capacity $c$ of the underlying material in the form [183]:

$$
T_{D}(z=0, t)=\int_{0}^{t} \theta\left(t^{\prime}\right) \cdot T\left(z=0, t-t^{\prime}\right) d t^{\prime}=\frac{2 Q}{\tau e \sqrt{\pi}}(\sqrt{t}-\sqrt{t-\tau})
$$

This is tracked via the time-dependent surface reflectivity change with a 643 $\mathrm{nm}$ continuous wave laser and a fast Si-photodiode. Details concerning such a measurement will be published elsewhere. Since the diameter of the pump pulse is much larger than the diameter of the probe pulse, the thermal transport can be seen as one-dimensional and perpendicular to the layers, allowing above analytical solution of the heat equation as long as the samples are thicker than the thermal penetration depth in order to avoid substrate effects [74]. The data sets for the different samples as well as their corresponding fits are plotted in Fig. 6.4. One can clearly see that for 340 interfaces the signal is strongest after laser pulse excitation meaning that heat accumulation at the surface is strongest and as a consequence, the thermal conductivity is lowest. But also the sample with 170 interfaces exhibits a smaller thermal conductivity than the pure $\mathrm{ZrO}_{2}$ sample, meaning that even a combination of a well conducting material as $\mathrm{W}\left(\kappa_{W} \sim 170 \mathrm{~W} / \mathrm{mK}\right)$ with an insulator as $\mathrm{ZrO}_{2}\left(\kappa_{\mathrm{ZrO}_{2}} \sim 2 \mathrm{~W} / \mathrm{mK}\right)$ can decrease the overall thermal conductivity of the whole material below the value of the insulating $\mathrm{ZrO}_{2}$ due to the interface effects. The combined conductivity $\kappa_{M L}$ of a $\mathrm{W} / \mathrm{ZrO}_{2}$ multilayer consisting of $N$ periods $\Lambda=d_{\mathrm{W}}+d_{\mathrm{ZrO}_{2}}$ with single layer thicknesses $d_{\mathrm{W}}$ and $d_{\mathrm{ZrO}}$ for $\mathrm{W}$ and $\mathrm{ZrO}_{2}$, respectively, and total thickness of the multilayer $D=N \Lambda$, can be described by the following equation as a resistor chain [148]:

$$
\kappa_{M L}=\frac{1}{\frac{x}{\kappa_{W}}+\frac{1-x}{\kappa_{Z r O}}+\frac{n}{G_{i}}} .
$$

This equation includes the contributions of the individual layers (relative layer thickness of the $\mathrm{W}$ layer is defined by $x=N d_{W} / D=d_{W} / \Lambda$ ) and the interface conductance $G_{i}$ with the interface density $n=2 N / D=2 / \Lambda$. The thermal conductivities of all the measured samples as well as the bulk values of $\mathrm{W}$ and $\mathrm{ZrO}_{2}$ are plotted in Fig. 6.5. First, one should notice that the measured value of the pure $\mathrm{ZrO}_{2}$ film agrees well with the literature bulk value. In the case of a homogeneous 


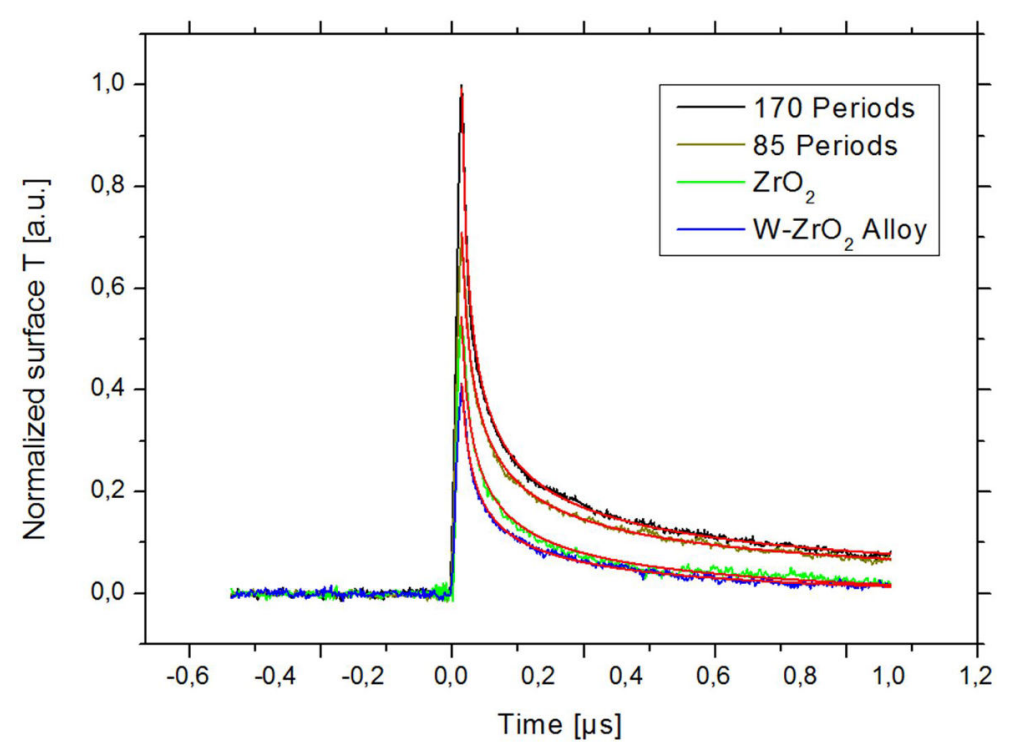

Figure 6.4.: Results of transient thermoreflectometry measurements on different multilayer samples as well as on a $\mathrm{ZrO}_{2}$ sample and an intermixed $\mathrm{W}-\mathrm{ZrO}_{2}$ alloy (normalized to the strongest temperature increase in the multilayer with 170 periods). Additionally, analytical solutions of the one-dimensional heat equation are fitted on the respective measurement curves (lines in red).

$\mathrm{W}-\mathrm{ZrO}_{2}$ alloy, where no boundary resistances exist, the above equation of the combined conductivity $\kappa_{m i x}$ without boundary resistances simplifies to

$$
\kappa_{\text {mix }}=\frac{1}{\frac{x}{\kappa_{W}}+\frac{1-x}{\kappa_{Z r O_{2}}}} .
$$

This strongly simplified equation, which neglects atomistic scattering processes and uses only bulk values of $\mathrm{W}$ and $\mathrm{ZrO}_{2}$, is included in Fig. 6.5 as a dashed line. Interestingly, the measured value of the intermixed $\mathrm{W}-\mathrm{ZrO}_{2}$ alloy sample lies very close to this line compared to other work on thermal conductivity of some other alloyed materials [256]. This indicates that its conductivity can be described in this special case as a mixture of the thermal conductivities through $\mathrm{ZrO}_{2}$ and $\mathrm{W}$, and interfaces play no significant role. In contrast to this, the thermal conductivities of both multilayer samples are significantly reduced, although the embedded W layers obey a high thermal conductivity. This is a clear indication that the interfaces in these layered samples must play a significant role. The role of the interfaces can be expressed by phonon scattering (due to interface roughness) and localization (due to reflection) but also by suppression of coherent phonon propagation modes due to detuning of individual layer thicknesses, which is likely to happen during 


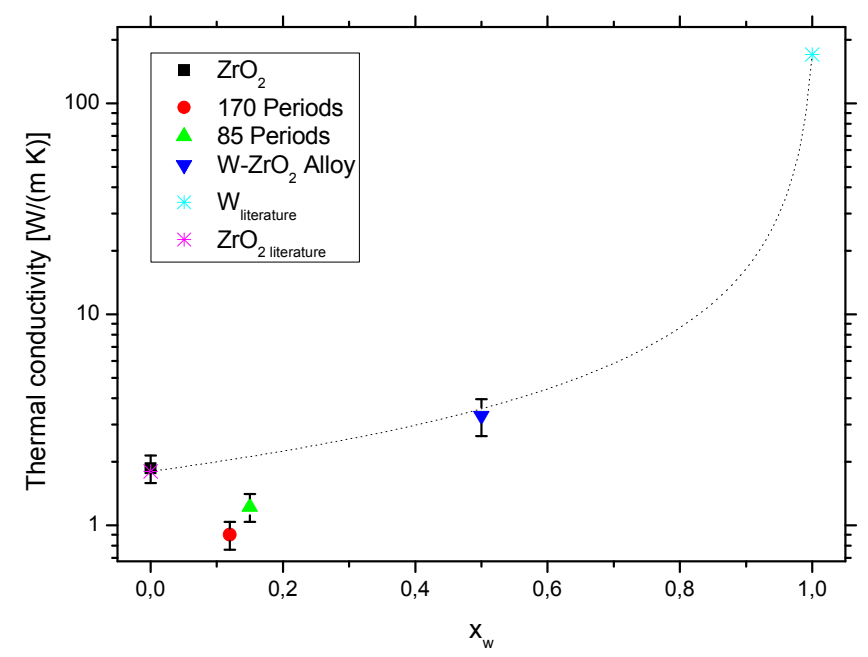

Figure 6.5.: Results of thermal conductivity measurements of a single $900 \mathrm{~nm} \mathrm{ZrO}_{2}$ layer, the two $\mathrm{W} / \mathrm{ZrO}_{2}$ multilayers with same overall thickness, but different interface density, and a fully intermixed $\mathrm{W}-\mathrm{ZrO}_{2}$ alloy. For comparison, a calculation for the thermal conductivity of interface-less compound mixtures of $\mathrm{ZrO}_{2}$ and $\mathrm{W}$ is drawn as a dashed line. Obviously, the multilayer samples feature a significantly lower thermal conductivity due to their high interface density.

the growth process $[164,165]$. All these effects are based upon the high interface density and lead to a reduction of the thermal conductivity, which can even be lower than the amorphous limit [9]. Due to the (experimental) knowledge of the interface density $\mathrm{n}$ and layer thicknesses, the thermal boundary conductance $G_{i}$ of the $\mathrm{W} / \mathrm{ZrO}_{2}$ interfaces can be estimated from the measurements of $\kappa_{M L}$ to a value of about $G_{i} \approx 6.3 \cdot \frac{10^{8} \mathrm{~W}}{m^{2} \mathrm{~K}}$. Thus, it can be concluded that the strong decrease of the overall thermal conductivity $\kappa_{M L}$ of both $\mathrm{W} / \mathrm{ZrO}_{2}$ multilayers even to a value of below $1 \mathrm{~W} / \mathrm{mK}$ is dominated by the high density of interfaces.

\subsection{Conclusion}

In summary, it has been shown that the laser deposited $\mathrm{W} / \mathrm{ZrO}_{2}$ multilayers fulfill two major requirements for high temperature applications such as thermal barrier coatings. They are thermally stable even at elevated temperatures up to $1300 \mathrm{~K}$ and their thermal conductivity can be significantly reduced (to a value of below $1 \mathrm{~W} / \mathrm{mK}$ ) compared to the bulk values of the individual components due to the high number of interfaces within the multilayers. 


\section{Acknowledgment}

We would like to thank Burkhard Roos for his support with the high-resolution TEM. This work was supported by the Deutsche Forschungsgemeinschaft (SFB 1073, project A02). 



\section{Further unpublished results}

Because not all TTR measurements could be published in time, the three most important additional projects were nevertheless included in this thesis as they further increase the scope of this work.

As this thesis was written in the surrounding of the CRC 1073 "atomic scale control of energy conversion", different successful collaborations have been established. Not only PLD-grown samples of the author have been measured in other groups, as already described in the previous chapters, but also thin films of other groups have been measured by the author with the newly established TTR method. Therefore, this addendum shall be used to show thermal conductivity measurements first on samples fabricated by atomic layer deposition (ALD) consisting mainly of $\mathrm{Al}_{2} \mathrm{O}_{3}$ and Pt produced by Christian Volkmann in the group of Sven Schneider. Second, a new polymer called PAzoPMA fabricated by RAFT polymerization by Dennis Hübner in the group of Phillipp Vana is measured. Finally, in order to complete the picture of this thesis, recent TTR results on the thermal conductivity of laser deposited W/PC multilayers prepared by the author are presented. Those multilayers and their corresponding measurements represent a final highlight of this thesis showing the lowest thermal conductivity of all materials in this work, which can be seen as a confirmation of the predicted results in chapter 4 .

\subsection{Thermal conductivity of atomic layer deposited thin films of $\mathrm{Pt}$ and $\mathrm{Al}_{2} \mathrm{O}_{3}$}

As a cooperation within the CRC 1073, the TTR method of this thesis has been used in order to measure thin film thermal conductivities of materials, which were prepared by ALD in another group. Christian Volkmann set up and implemented a new ALD reactor in the group of Sven Schneider with which he fabricated thin films consisting of $\mathrm{Pt}$ and $\mathrm{Al}_{2} \mathrm{O}_{3}$. Since the growth rates with the ALD method are comparatively small, only a very limited amount of 
samples could be measured. However, already those showed a strong reduction in thermal conductivity compared to a crystalline $\mathrm{Al}_{2} \mathrm{O}_{3}$ substrate, which was used as comparison. All in all, three samples have been investigated with TTR. The sample ALD 1, which is a structure consisting of $400 \mathrm{~nm} \mathrm{Al}_{2} \mathrm{O}_{3}$ atomic layer deposited onto $200 \mathrm{~nm} \mathrm{SiO}$, the sample ALD 2, which represents a multilayer of $\mathrm{Pt}$ and $\mathrm{Al}_{2} \mathrm{O}_{3}$ that is shown in figure 7.1, as well as the reference sample consisting of crystalline $\mathrm{Al}_{2} \mathrm{O}_{3}$.

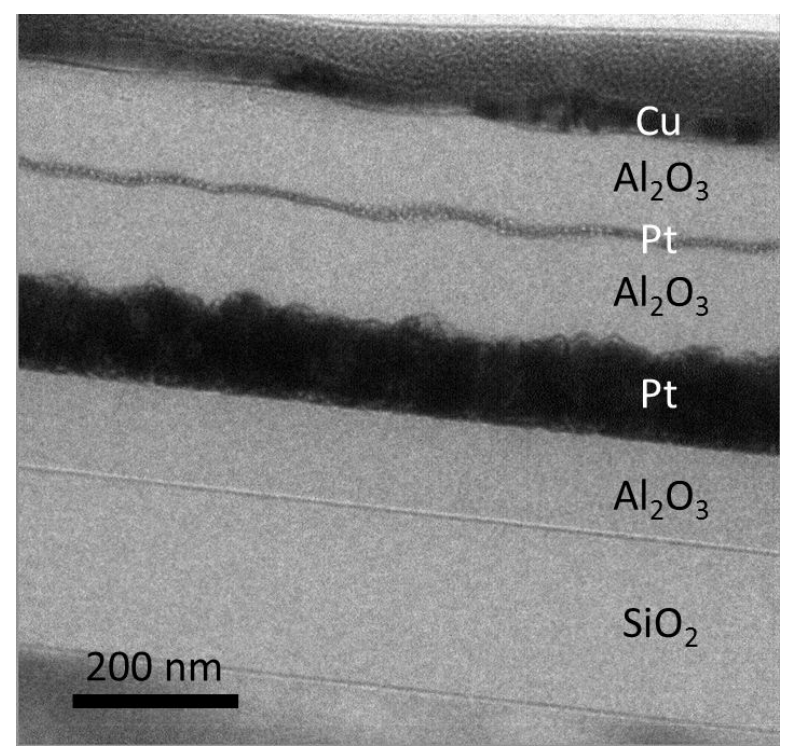

Figure 7.1.: Transmission electron micrograph of a cross-section through the sample ALD 2 , which consist mainly of $\mathrm{Pt}$ and $\mathrm{Al}_{2} \mathrm{O}_{3}$. The individual layers are marked within the figure. It can be clearly seen that the Pt layers are very rough and seem to consist of clusters with several nm diameter.

In contrast to the reference sample, the atomic layer deposited $\mathrm{Al}_{2} \mathrm{O}_{3}$ is amorphous, which could be determined in electron diffraction experiments using a TEM by the author with the help of Timo Wuttke, as well as in X-ray diffraction experiments conducted by the author.

TTR experiments have been prepared by depositing again $50 \mathrm{~nm} \mathrm{Cu}$ on top of all three samples using PLD. Afterwards, thermal conductivities were measured by TTR. The measurement curves and their corresponding fits are shown in figure 7.2. As expected, the crystalline $\mathrm{Al}_{2} \mathrm{O}_{3}$ reference curve shows the lowest heating effect at the surface and conducts thermal energy efficiently away. The evaluation of TTR data gave the result of a thermal conductivity around $46 \mathrm{~W} / \mathrm{mK}$, which is close to the literature value of $42.5 \mathrm{~W} / \mathrm{mK}$ [247]. In contrast to this, the samples produced with ALD show a very much lower thermal conductivity. Already sample ALD 1 had a measured thermal conductivity of only $2.8 \mathrm{~W} / \mathrm{mK}$, which 
is in marked contrast to the pure $\mathrm{Al}_{2} \mathrm{O}_{3}$. This at the first sight contradictory result can be understood by considering the fact that the $\mathrm{Al}_{2} \mathrm{O}_{3}$ in this sample grew amorphously by ALD, which reduces the thermal conductivity as explained in chapter 2. Derived from the TTR result, one can approximate the thermal conductivity of the amorphous $\mathrm{Al}_{2} \mathrm{O}_{3}$ fabricated by this ALD method to a value around $6 \mathrm{~W} / \mathrm{mK}$. Moreover, apart from the $400 \mathrm{~nm} \mathrm{Al}_{2} \mathrm{O}_{3}$ also a $200 \mathrm{~nm} \mathrm{SiO}_{2}$ layer was part of this sample. Due to the fact that $\mathrm{SiO}_{2}$ has a very low thermal conductivity of only about $1.3 \mathrm{~W} / \mathrm{mK}$, the whole thermal conductivity deviates so much from the crystalline $\mathrm{Al}_{2} \mathrm{O}_{3}$.

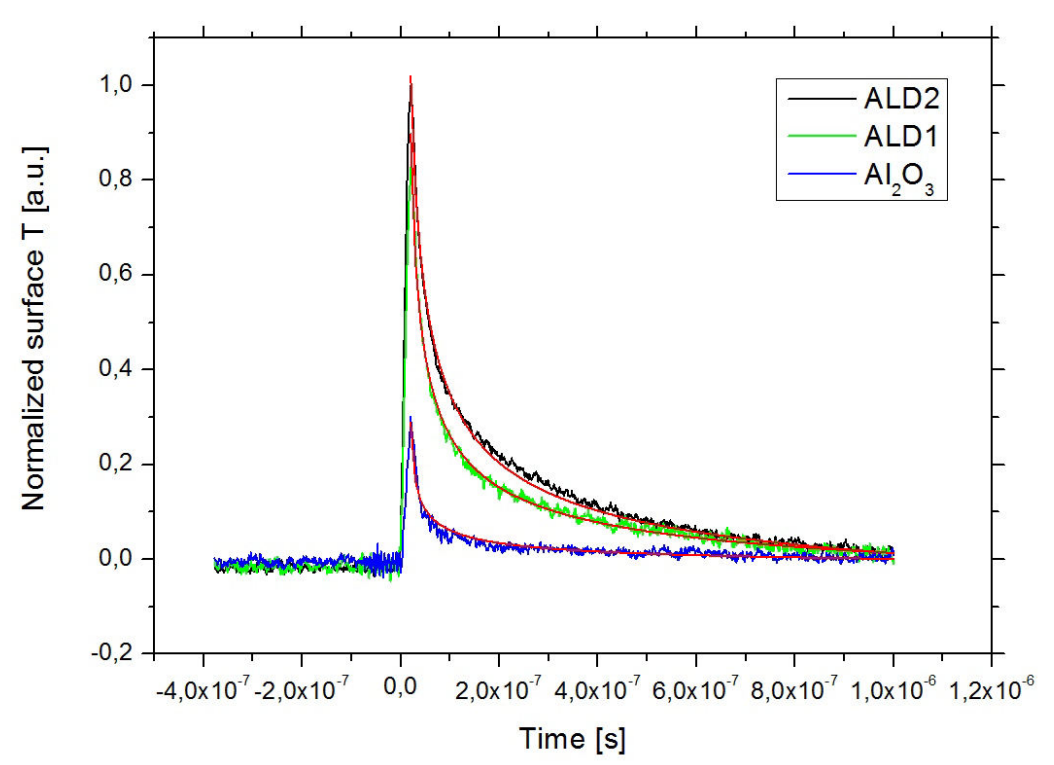

Figure 7.2.: Results of thermal conductivity measurements of three samples consisting mainly or partially of $\mathrm{Al}_{2} \mathrm{O}_{3}$. In the case of the pure and crystalline $\mathrm{Al}_{2} \mathrm{O}_{3}$, the deposited heat is decayed quickly at the surface. In contrast to this, the heating effect in both samples prepared by ALD is stronger, which can be attributed to the fact that $\mathrm{Al}_{2} \mathrm{O}_{3}$ in this case is amorphous and that a $200 \mathrm{~nm}$ thick $\mathrm{SiO}_{2}$ layer is also measured. Comparing both ALD samples, the one with Pt layers shows a lower thermal conductivity probably because of the great diffuse mismatch between $\mathrm{Pt}$ and $\mathrm{Al}_{2} \mathrm{O}_{3}$.

Comparing this result to the measurements of the sample ALD 2, we see a further reduction effect of thermal conductivity to a value of about $1.6 \mathrm{~W} / \mathrm{mK}$ although Pt has been added, which has a significantly higher thermal conductivity of ca. $72 \mathrm{~W} / \mathrm{mK}$ [257]. This effect could again be explained by thermal boundary resistances at the interfaces. Due to the great roughness of the layers, probably the diffuse mismatch model is the best to describe phonon propagation across the interface as was discussed in chapter 2 [86]. Here, the difference in phonon density of states in the materials at each side of the interface is most important and a great 
difference leads to a low interface conductance. Regarding the phonon density of states in $\mathrm{Pt}$, we see two maxima ca. at $2.7 \mathrm{THz}$ and $5.5 \mathrm{THz}$. For frequencies above $6 \mathrm{THz}$ the phonon density of states becomes zero in Pt [258]. In contrast to this, the phonon density of states for $\mathrm{Al}_{2} \mathrm{O}_{3}$ is non-zero even up to $27 \mathrm{THz}$ with its highest peak around $23 \mathrm{THz}$ [259]. From this small overlap in phonon density of states between the two materials, it becomes apparent that phonons in wide frequency ranges in $\mathrm{Al}_{2} \mathrm{O}_{3}$ cannot propagate in $\mathrm{Pt}$ and that phonons with characteristic frequencies in $\mathrm{Pt}$ cannot excite a broad spectrum of phonons in $\mathrm{Al}_{2} \mathrm{O}_{3}$. In literature, boundary resistance for the related $\mathrm{Al}_{2} \mathrm{O}_{3} / \mathrm{Pb}$ system was quantified by $1.8 \cdot 10^{-6} \mathrm{~m}^{2} \mathrm{~K} / \mathrm{W}$, which is also plausible for the $\mathrm{Al}_{2} \mathrm{O}_{3} / \mathrm{Pt}$ system and correlates well with the results of the TTR experiment [156]. Since in this sample only two Pt layers were present, the effect of interface thermal boundaries could not be strong enough to serve alone for the thermal conductivity reduction.

Another reason for the overall low thermal conductivity could be the existence of the $\mathrm{SiO}_{2}$ layer as mentioned above, but also a reduced thermal conductivity of the Pt layers that seem to form unnatural clusters, visible in the transmission electron micrograph 7.1. Here, possible contaminations during the ALD process or precursor remnants could lead to a reduction of the thermal conductivity, density or heat capacity of Pt below the original bulk value, which would contribute to the overall reduction in measured thermal conductivity.

\subsection{TTR-measurements on the first PAzoPMA polymer}

In the sense of measuring low thermal conductivity materials, also TTRmeasurements on polymers have been desired, which are more complicated to conduct due to their comparatively easy destructibility with high fluence laser pulses. Apart from measurements on pulsed laser deposited polymer layers of which the most important ones shall be presented later, also a polymer sample from the group of Phillipp Vana, fabricated by Dennis Hübner, has been measured. This special polymer is called PAzoPMA and the group around Philip Vana was the first to succeed in synthesizing it [260]. After careful deposition of the $50 \mathrm{~nm} \mathrm{Cu}$ transducer layer for TTR as explained in chapter 5, by the author, this specialized material could be investigated with the TTR method. Therefore, especially low laser energies were used in order not to destroy the sample. The result of this measurement as well as a corresponding fit is shown in figure 7.3. 


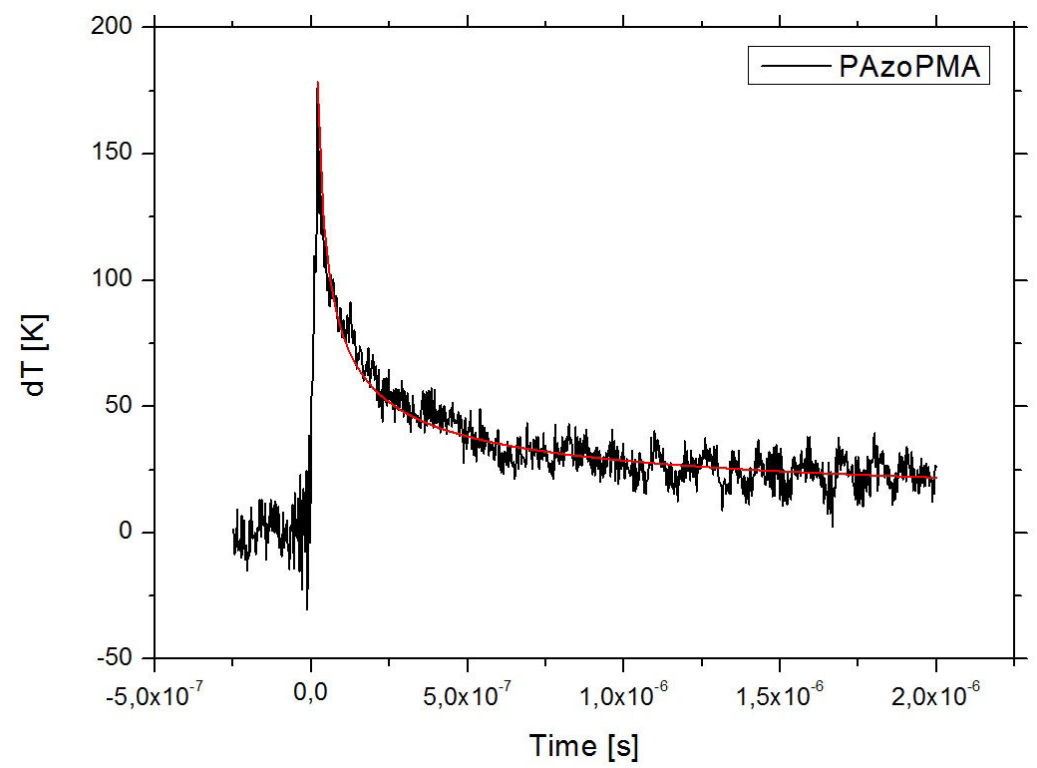

Figure 7.3.: The TTR method has been used for thermal conductivity measurements on a PAzoPMA sample. The result is a typical thermal conductivity of a polymer of $0.2 \mathrm{~W} / \mathrm{mK}$ but has to be treated with caution since PAzoPMA is a relatively unknown polymer with therefore undescribed properties.

The time-resolved reflectivity spectrum shows a curve shape, which could be evaluated according to the routine of chapter 5. Approximating the density and heat capacity of this new polymer with the bulk values of the well-known polymer PMMA, one gets a thermal conductivity of ca. $0.2 \mathrm{~W} / \mathrm{mK}$ [261]. This result is very plausible, since it is a typical value for a polymer [261,262].

The specialty of this PAzoPMA is that it is UV light-responsive, making it possible to change its isomerization and thereby switch its molecular ordering. This could possibly provide a future path to light-induced active control of thermal conductivity making future TTR measurements on such materials very promising.

\subsection{Thermal conductivity measurements on W/PC multilayers}

Finally, TTR measurements on W/PC multilayer were important for this thesis. According to the theory presented in chapter 2 those are supposed to have a very low thermal conductivity due to the great acoustic mismatch at the interfaces. Moreover, first experimental and theoretical evidence for thermal conductivity values below $0.1 \mathrm{~W} / \mathrm{mK}$ were found already in chapter 4 , which should be 
confirmed by TTR-measurements. Therefore, high effort has been expended in order to fabricate three samples consisting of the very different materials $\mathrm{W}$ and PC. For this experiment, the interface density should be maximized, meaning that complex studies on minimal layer thicknesses in the W/PC multilayer system had to be conducted. The result was a sample with overall thickness of $1.2 \mu \mathrm{m}$ consisting of 800 interfaces between $2.5 \mathrm{~nm}$ thick PC layers and only $0.5 \mathrm{~nm}$ thick W layers, which represents an interface density of $0.67 \mathrm{~nm}^{-1}$. For comparison, a second $\mathrm{W} / \mathrm{PC}$ multilayer sample of almost the same overall thickness, but only of half the amount of interfaces was pulsed laser deposited. In order to obtain information about the thermal conductivity of pulsed laser deposited PC, a third sample, which was a monolayer of ca. $1.2 \mu \mathrm{m}$ PC was fabricated and measured.

After adding the $\mathrm{Cu}$-top layer, all three samples could be measured by TTR giving the curves shown with their fits in figure 7.4. The outcome of these measurements

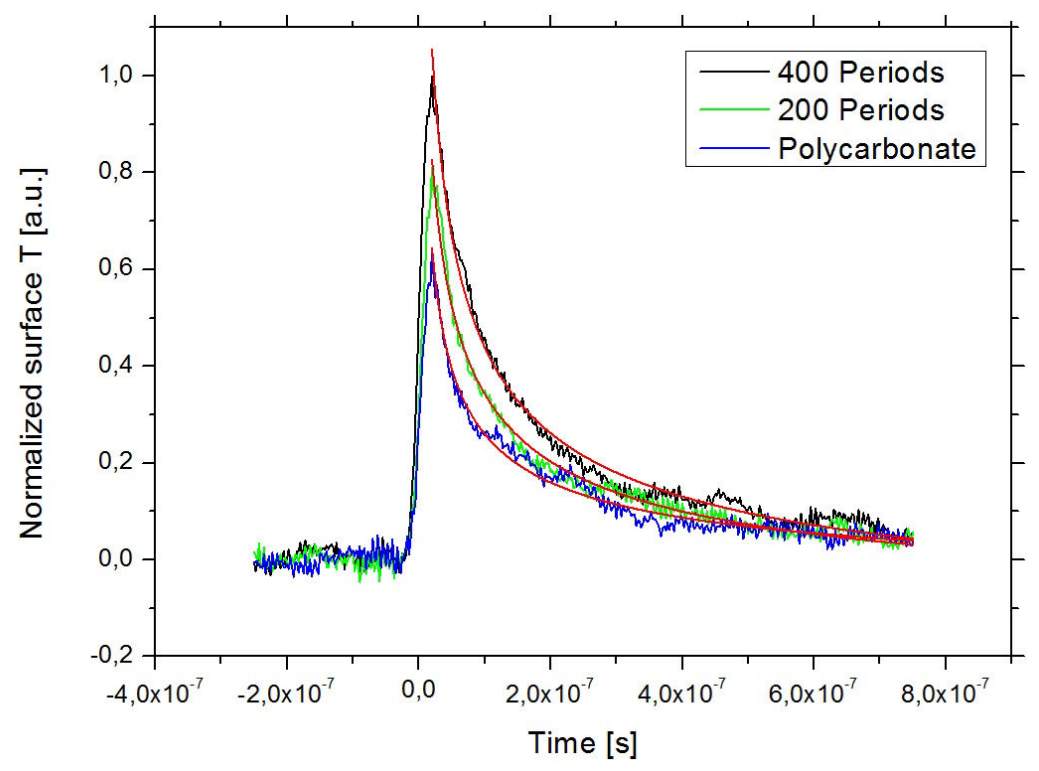

Figure 7.4.: Pulsed laser deposited PC as well as two W/PC multilayers with 200 and 400 periods, respectively have been measured by TTR. While already PC has a very low thermal conductivity, multilayers of W and PC could decrease this value significantly due to their thermal boundary resistances.

showed for the pulsed laser deposited PC sample a value of $\kappa_{P C-P L D} \approx 0.4 \mathrm{~W} / \mathrm{mK}$ which is significantly higher than the literature value for bulk PC $\kappa_{P C} \approx 0.2 \mathrm{~W} / \mathrm{mK}$ $[214,263]$. The reason for this obvious deviation could be a change in polymeric properties during laser ablation, which changes the microstructure for instance by an increase of cross-linking [264]. Due to this structural change, the pulsed laser deposited polymer becomes harder than its original target material, which 
could be described in this work in chapter 4 and 3. There, it was found that the E-modulus of laser deposited polymer utilized for this experiment has a value of $E_{P C-P L D} \approx 7 \mathrm{GPa}$ in contrast to the bulk value of $E_{P C} \approx 2 \mathrm{GPa}$ [265]. This increase in hardness by a factor of about three could also be the reason for the higher thermal conductivity of pulsed laser deposited PC. Utilizing a rough estimation according to which the phonon velocity scales with $v \propto \sqrt{E}$, the thermal conductivity described with equation 2.5, namely $\kappa=\frac{1}{3} \mathrm{cvl}$, would also scale with $\kappa \propto \sqrt{E}$ [266]. This estimation results in a thermal conductivity for laser deposited PC of about $\kappa_{P C-P L D} \approx 0.37 \mathrm{~W} / \mathrm{mK}$, which is very close to the measured value.

Continuing the analysis of the two W/PC multilayers with the adjusted bulk value for laser deposited PC, one gets a strong reduction of thermal conductivity below the value of PC already in the sample with an interface density of $0.33 \mathrm{~nm}^{-1}$. Here, a value of $0.16 \mathrm{~W} / \mathrm{mK}$ was measured, which is also lower than the literature value for unmodified PC and thereby an amazing result. However, the second W/PC multilayer sample could surpass this result, since here the individual layer thicknesses were reduced even more, resulting in an interface density that was twice as high. This sample showed a thermal conductivity of only $0.09 \mathrm{~W} / \mathrm{mK}$, which is an ultra-low value for fully dense materials and which is, to the knowledge of the author, undercut only by the thermal conductivity $\kappa\left(W S e_{2}\right)=0.05 \mathrm{~W} / \mathrm{mK}$ of the famous mono-atomically ordered WSe $\mathrm{W}_{2}$ crystal [142].

Combining the measurements on both $\mathrm{W} / \mathrm{PC}$ multilayer samples, one can estimate the thermal boundary conductance to a value of around $G_{W / P C} \approx 8 \cdot 10^{7} \mathrm{~W} /\left(\mathrm{m}^{2} \mathrm{~K}\right)$ which is higher, but still in the same order of magnitude as expected in chapter 4. The reason for this enlarged value could be again the modification of the polymer, making the acoustic mismatch lower due to the increase of PC hardness accompanied by the increase in sound velocity. Nonetheless, this is still a quite low value for interfacial thermal conductance and much lower than the value for $\mathrm{W} / \mathrm{Al}_{2} \mathrm{O}_{3}$, which is ca. $2.6 \cdot 10^{8} \mathrm{~W} /\left(\mathrm{m}^{2} \mathrm{~K}\right)$ or $\mathrm{W} / \mathrm{ZrO}_{2}$, which is ca. $6.3 \cdot 10^{8} \mathrm{~W} /\left(\mathrm{m}^{2} \mathrm{~K}\right)[39]$.

All in all, these measurements on $\mathrm{W} / \mathrm{PC}$ prove that it is a material combination suitable for fabrication of ultra-low thermal conductivity multilayer materials. The challenges of manufacturing those with high quality and low layer thicknesses have been faced in this thesis. Thus, such structures were presented here for the first time as well as measured for the first time concerning their thermal conductivity with an excellent result. 



\section{Conclusion}

This present thesis combines research published in four different peer-reviewed papers. It represents work in the fields of phononics and thermal conductivity research, as well as materials physics in terms of fabrication and characterization of nanoscale multilayers, but also measurement method development and application. The backbone of this work are pulsed laser deposited thin films, especially multilayers. Those nanoscale structures have been investigated mainly in terms of cross-plane thermal conductivity and therefore also concerning phonon transport and their structure. The pronounced goal was to minimize the thermal conductivity in multilayer systems. Hence, gathering understanding about phononic heat transport was important. In order to achieve that goal, investigations had to be conducted covering all the necessary methods, like pulsed laser deposition (PLD), transient thermoreflectometry (TTR), X-ray reflectivity, X-ray diffraction, transmission and scanning electron microscopy as well as ultrafast optical pumpprobe spectroscopy, which was performed in a collaboration with Henning Ulrichs and Markus Münzenberg.

The starting point from simple bilayers towards more complex multilayers is explained in chapter 3, where phonon localization in thin, layered structures was found. For this endeavor, thin films consisting of $\mathrm{Cu}$ and polycarbonate (PC) or W and $\mathrm{Si}$ were prepared by PLD, because they exhibit a large acoustic mismatch. They were compared in terms of phonon transport with sputtered material combinations, namely applied functional materials for heat-assisted magnetic recording and ultrathin metal-oxide multilayers. It was shown that especially high acoustic mismatch material combinations exhibit strong reflection and re-reflection of phonons at interfaces, by which phonon modes were localized as standing waves within the layers. In the $\mathrm{Cu} / \mathrm{PC}$ system, where phonon transmission probabilities across the interfaces were found to be very low, standing phonon waves with a long life-time of up to $500 \mathrm{ps}$ could be detected. This is a sophisticated approach towards phonon mean free path reduction by boundary scattering and reflection and can be seen as an experimental validation of different phonon transport theories, like 
the acoustic or diffuse mismatch model $[59,60,86]$, but also as a very descriptive example for phonon localization that explains, how heat transport can be hindered. In addition to this, it was achieved to tune the frequency of the standing waves over a wide range by using different layer thicknesses. Here, phonon frequencies up to $360 \mathrm{GHz}$ were found, which can almost be seen as thermal frequencies. By further reducing the lattice period, frequencies above $1 \mathrm{THz}$ might also be accessible in the future, making such structures even more important for thermal transport suppression.

All in all, this first work identified PLD as an ideal method for preparing phonon confining thin films. Especially the ability of combining very thin layers of different materials, such as hard and heavy metals with soft and lightweight polymers is outstanding and very important for new concepts of thermal insulation.

As outlined in chapter 4, such material combinations could be grown with high quality after an improvement of the PLD-setup by the author. The materials of choice were $\mathrm{W}$ and PC and to the knowledge of the author, this thesis is the first to describe W/PC multilayers because of the challenges in producing such. Since W is a well-established material, even for PLD, mostly the PC had to be investigated before starting multilayer preparation. It could be shown that laser deposited PC remains its original molecular bonds over a wide range of laser fluences from $30 \mathrm{~mJ} / \mathrm{cm}^{2}$ up to $250 \mathrm{~mJ} / \mathrm{cm}^{2}$ as confirmed by NEXAFS measurements in collaboration with Klaus Mann and Matthias Müller from the Laser Laboratorium Göttingen. However, measurements of PC-hardness showed a significant increase in hardness with increasing laser fluence, making this polymer adjustable in its mechanical properties. After finding also a minimum in roughness with laser fluence, multilayers could be prepared with small individual layer thickness, low roughness and high acoustic mismatch, making such material combination an appropriate candidate for further phononic experiments.

Using fs-pump-probe reflectivity measurements, first, on periodic multilayers of $\mathrm{W}$ and PC, strong phonon localization and phonon mode confinement could be confirmed as expected according to band structure calculations. This result matches different findings on phononic crystals in literature and again increases their scope by manipulating high frequency phonons above $300 \mathrm{GHz}$ in a new material combination $[229,232]$. From the pump-probe measurements in this work it became clear that coherent phonon modes with appropriate frequencies can pass through a periodic multilayer as Bloch-like eigenmodes. Thereof, the idea resulted to terminate the propagation with small deviations from the periodic structure. 
Hence, aperiodic structures were designed that filter even more phonon modes, which lead to the total absence of detectable transmitting coherent phonons.

These experimental results could be taken as an explanation for a reduction effect in thermal conductivity of randomly stacked multilayers that was found in theoretical calculations in literature $[164,165]$.

In order to measure thermal conductivities in this thesis, an own measurement setup had to be conceived and implemented. Because of the high spectrum of samples that can be produced by PLD, a new and versatile implementation of the well-known transient thermoreflectance (TTR) scheme was realized, which was described and tested in chapter 5. For this novel implementation, a pulsed $\mathrm{KrF}$ excimer laser was chosen for heating that runs at a wavelength of $248 \mathrm{~nm}$ and with pulse durations of $20 \mathrm{~ns}$. The change in temperature after this heating pulse is measured via a $643 \mathrm{~nm}$ continuous-wave laser that probes the surface reflectivity. In order to obtain a usable measurement signal and optimize the signal to noise ratio, different metal transducers with different thicknesses were tested for this method with the result that $50 \mathrm{~nm}$ of $\mathrm{Cu}$ are suited best to measure the reflectivity change.

This time-dependent change in reflectivity was then translated into a timedependent change in temperature via the coefficient of thermoreflectometry. In order to extract thermal conductivity data from the measured change in temperature, the heat equation was solved with respect to the given parameters of the heating laser. The resulting approximated analytical formalism was utilized for data collection and interpretation. For measuring thin film thermal conductivities, which was one goal of this thesis, a special treatment for multilayers was developed as a quasi-material approach to density, heat capacity and thermal conductivity that takes into account diffusive heat transport as a resistor chain. This here developed procedure was used in order to determine the thermal conductivity of several different bulk materials as well as various thin film systems. An overview of the results is depicted in figure 8.1, where the most important measurements of this thesis are plotted in comparison with important literature values. Since the here presented implementation with such a laser combination is unique in the literature, scarcely any literature values or descriptions for this setup existed. After great efforts in designing, implementing, testing and improving this method, a comparatively handy, versatile method with high throughput capabilities has been established that represents a novel contribution to the field of thermal conductivity measurement techniques. 


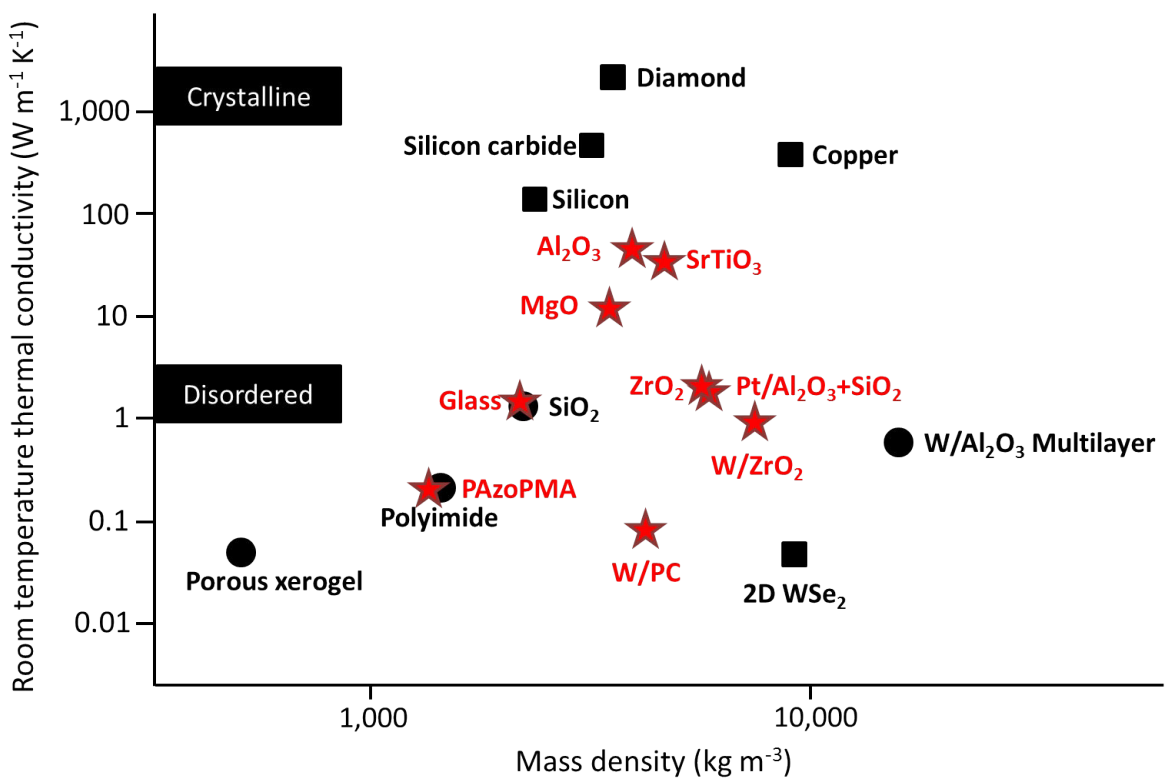

Figure 8.1.: Comparison of thermal conductivity values that were measured in this thesis (red stars) with some representative literature values (black squares and circles) at room temperature. Of special interest are the data points for $\mathrm{W} / \mathrm{ZrO}_{2}$ and $\mathrm{W} / \mathrm{PC}$ in comparison to the ultra-low literature values of $\mathrm{W} / \mathrm{Al}_{2} \mathrm{O}_{3}$ and the two-dimensionally layered $\mathrm{WSe}_{2}[39,142]$. Concerning $\mathrm{W} / \mathrm{ZrO}_{2}$, this material combination has a much higher thermal stability than $\mathrm{W} / \mathrm{Al}_{2} \mathrm{O}_{3}$ while being in the same thermal conductivity region. In the case of nanoscale $\mathrm{W} / \mathrm{PC}$ multilayers that were presented first in this thesis a tremendously low value of thermal conductivity was achieved even without the need of monoatomically stacking of layers. (Figure adapted from [35])

One of the most important measurements with this TTR setup within the scope of this thesis is described in chapter 6. Here, this method is applied to laser deposited multilayers of $\mathrm{W}$ and $\mathrm{ZrO}_{2}$. This material combination has been chosen on the one hand because $\mathrm{ZrO}_{2}$ is a prominent thermal barrier material and features a low thermal conductivity and on the other hand because both materials have a very high melting point and are therefore thermally very stable. In order to decrease the thermal conductivity of $\mathrm{ZrO}_{2}$, it has been combined together with $\mathrm{W}$ in nanoscale multilayers. As described in chapter 2, such material combination poses strong resistance to heat flow because of the boundary resistances at every interface. For the fabrication of such multilayers, layer thicknesses have been designed in order to benefit from the intrinsic low thermal conductivity of $\mathrm{ZrO}_{2}$, while $\mathrm{W}$ (that features a bulk thermal conductivity, which is almost two orders of magnitude higher than that of $\mathrm{ZrO}_{2}$ ) was only used for ultra-thin blocking layers. In this way, the bulk thermal conductivity is dominated by the low value of $\mathrm{ZrO}_{2}$, while $\mathrm{W}$ layers with thicknesses down to $0.6 \mathrm{~nm}$ have been fabricated as phonon blocking interlayers. 
By this approach, the thermal conductivity could be decreased below $1 \mathrm{~W} / \mathrm{mK}$, which is significantly below the already low value of $\mathrm{ZrO}_{2}$ and also lower than a measured alloy value in this system. Moreover, it could be shown by repeated XRR measurements after annealing at high temperatures that the laser deposited $\mathrm{W} / \mathrm{ZrO}_{2}$ multilayers show a high thermal stability even up to $1300 \mathrm{~K}$. This means that this material combination fulfills the two most important requirements for many high-temperature applications, namely a high thermal stability together with a low thermal conductivity.

This result is very important because many low thermal conductivity multilayer materials do not feature this outstandingly high thermal stability, while for highly stable thermal barrier materials there is plenty of space for improvement by nanostructuring these in terms of multilayers. One decade ago Costescu et al. have written in their world-famous Science paper: 'Applications of nanolaminates as thermal barriers at temperatures higher than $1000^{\circ} \mathrm{C}$ would require the development of material interfaces that satisfy the conflicting demands of low thermal conductance and exceptional thermal stability' [39]. These conflicting demands have been fulfilled in the multilayers fabricated and investigated in this thesis.

Another highlight of this thesis is shown in chapter 7, where first TTR measurements on the switchable polymer PAzoPMA, atomic layer deposited $\mathrm{Pt} / \mathrm{Al}_{2} \mathrm{O}_{3}$ thin films and pulsed laser deposited W/PC multilayers are presented. Here, especially the measurements on $\mathrm{W} / \mathrm{PC}$ multilayers are of great importance for this work. This material combination was not only fabricated for the first time in this thesis, but also investigated in terms of its cross-plane thermal conductivity for the first time with the newly established TTR method. This alone is already a strong result, because this material combination can only be grown with PLD in this quality and measuring its thermal conductivity is highly nontrivial because of the easy destructibility of the polymer, which excludes some measurement methods, for instance the 3-omega method. Apart from this, the W/PC multilayers produced here featured an ultra-low thermal conductivity value of $0.09 \mathrm{~W} / \mathrm{mK}$, which constitutes a peculiar result, since it lies in the same order of magnitude as the unprecedented value of the monoatomically layered WSe ${ }_{2}$ crystal [142].

All in all, this thesis has managed to reach its goal of reducing thermal conductivity in laser deposited multilayers. This is shown in figure 8.1, were the gained results of this thesis are compared with other outstanding material combinations as well as important bulk values.

In order to achieve this result, phonon transport in bilayers and multilayers was 
investigated and phonon propagation was studied for the first time in the high acoustic mismatch material combination $\mathrm{W} / \mathrm{PC}$, where strong confinement in periodic and especially in aperiodic multilayers could be shown. With a novel TTR measurement method that has been implemented in the scope of this thesis, the reduction of thermal conductivity in highly stable thermal barrier $\mathrm{W} / \mathrm{ZrO}_{2}$ multilayers as well as in ultra-low thermal conductivity W/PC multilayers could be demonstrated.

Altogether, this thesis represents advancement in thermal conductivity research and provides a foundation for possible future thermal barrier applications. 


\section{Bibliography}

[1] Kittel Charles: Introduction to Solid State Physics. 8th ed. Hoboken, NJ : Wiley 2005

[2] Неснт Eugene: Optics. 4th ed. Reading, Mass : Addison-Wesley 2002

[3] Zangwill Andrew: Modern Electrodynamics. Cambridge : Cambridge University Press 2013

[4] Maldovan Martin: Sound and Heat Revolutions in Phononics. Nature 503 (2013) November, S. 209-217

[5] Chen Gang: Nanoscale Energy Transport and Conversion: A Parallel Treatment of Electrons, Molecules, Phonons, and Photons. Oxford ; New York : Oxford University Press 2005 (MIT-Pappalardo series in mechanical engineering)

[6] Nipko J. C., Loong C.-K., Balkas C. M., Davis R. F.: Phonon Density of States of Bulk Gallium Nitride. Applied Physics Letters 73 (1998), S. 34

[7] Chen Gang: Phonon Heat Conduction in Nanostructures. International Journal of Thermal Sciences 39 (2000) April, S. 471-480

[8] Narayana Supradeep, Sato Yuki: Heat Flux Manipulation with Engineered Thermal Materials. Physical Review Letters 108 (2012) Mai

[9] Popa Bogdan-Ioan, Zigoneanu Lucian, Cummer Steven A.: Experimental Acoustic Ground Cloak in Air. Phys. Rev. Lett. 106 (2011) Juni, S. 253901

[10] Deymier Pierre A.: Introduction to Phononic Crystals and Acoustic Metamaterials. In: Deymier Pierre A. (Hrsg.): Acoustic Metamaterials and Phononic Crystals Bd. 173. Berlin, Heidelberg : Springer Berlin Heidelberg 2013 S. 1-12

[11] Kushwaha M. S., Halevi P., Dobrzynski L., Djafari-Rouhani B.: Acoustic Band Structure of Periodic Elastic Composites. Phys. Rev. Lett. 71 (1993) September, S. 20222025

[12] Venkatasubramanian Rama, Sirvola Edward, Colpitts Thomas, O'Quinn Brooks: Thin-Film Thermoelectric Devices with High Room-Temperature Figures of Merit. Nature 413 (2001) Oktober, S. 597-602 
[13] Harman T. C., Taylor P. J., Walsh M. P., LaForge B. E.: Quantum Dot Superlattice Thermoelectric Materials and Devices. Science 297 (2002) September, S. 2229

[14] Cheng Ying, Yang Fan, Xu Jian Y., Liu Xiao J.: A Multilayer Structured Acoustic Cloak with Homogeneous Isotropic Materials. Applied Physics Letters 92 (2008)

[15] An Kyongjun, Ravichandran Kakkaveri S., Dutton Rollie E., Semiatin S. L.: Microstructure, Texture, and Thermal Conductivity of Single-Layer and Multilayer Thermal Barrier Coatings of Y2O3-Stabilized ZrO2 and Al2O3 Made by Physical Vapor Deposition. Journal of the American Ceramic Society 82 (2004) Dezember, S. 399-406

[16] Zhang Shu, Xia Chunguang, Fang Nicholas: Broadband Acoustic Cloak for Ultrasound Waves. Physical Review Letters 106 (2011) Januar

[17] Liang B., Guo X. S., Tu J., Zhang D., Cheng J. C.: An Acoustic Rectifier. Nature Materials 9 (2010) Dezember, S. 989-992

[18] Boechler N., Theocharis G., Daraio C.: Bifurcation-Based Acoustic Switching and Rectification. Nat Mater 10 (2011) September, S. 665-668

[19] Meric Inanc, Han Melinda Y., Young Andrea F., Ozyilmaz Barbaros, Kim Philip, ShePARd Kenneth L.: Current Saturation in Zero-Bandgap, Top-Gated Graphene FieldEffect Transistors. Nat Nano 3 (2008) November, S. 654-659

[20] Авнат A.: Low Temperature Latent Heat Thermal Energy Storage: Heat Storage Materials. Solar Energy 30 (1983), S. 313-332

[21] Khudhair Amar M., Farid Mohammed M.: A Review on Energy Conservation in Building Applications with Thermal Storage by Latent Heat Using Phase Change Materials. Energy Conversion and Management 45 (2004) Januar, S. 263-275

[22] Pernot G., Stoffel M., Savic I., Pezzoli F., Chen P., Savelli G., Jacquot A., Schumann J., Denker U., Mönch I., Deneke Ch., Schmidt O. G., Rampnoux J. M., Wang S., Plissonnier M., Rastelli A., Dilhaire S., Mingo N.: Precise Control of Thermal Conductivity at the Nanoscale through Individual Phonon-Scattering Barriers. Nature Materials 9 (2010) Juni, S. 491-495

[23] Koblinger O., Mebert J., Dittrich E., Döttinger S., Eisenmenger W., Santos P. V., Ley L.: Phonon Stop Bands in Amorphous Superlattices. Physical Review B 35 (1987) Juni, S. 9372-9375

[24] Heremans Joseph P.: Thermoelectricity: The Ugly Duckling. Nature 508 (2014) April, S. $327-328$ 
[25] HTTPS://WWW.BMWI.DE/BMWI/REDAKTION/PDF/E/ENERGIESTATISTIKENGRAFIKEN,PROPERTY=PDF,BEREICH $=$ BMWI2012,SPRACHE=DE,RWB=TRUE.PDF: Energiedaten: Gesamtausgabe. 2014 (Arbeitsgemeinschaft Energiebilanzen (AGEB), Bundesverband der Energie- und Wasserwirtschaft (BDEW))

[26] Schmidt M, Schwertfeger F: Applications for Silica Aerogel Products. Journal of NonCrystalline Solids 225 (1998) April, S. 364-368

[27] Tyagi Vineet V., Buddhi D.: PCM Thermal Storage in Buildings: A State of Art. Renewable and Sustainable Energy Reviews 11 (2007) August, S. 1146-1166

[28] Schutz J.B: Properties of Composite Materials for Cryogenic Applications. Cryogenics 38 (1998) Januar, S. 3-12

[29] Nuuguna J., Pielichowski K.: Polymer Nanocomposites for Aerospace Applications: Properties. Advanced Engineering Materials 5 (2003) November, S. 769-778

[30] Baetens Ruben, Jelle Bjørn P., Gustavsen Arild: Aerogel Insulation for Building Applications: A State-of-the-Art Review. Energy and Buildings 43 (2011) April, S. 761769

[31] Nolas George S., Sharp Jeffrey, Goldsmid H. J.: Thermoelectrics Basic Principles and New Materials Developments. Berlin, Heidelberg : Springer Berlin Heidelberg 2001

[32] Tritt Terry M., Subramanian M. A.: Thermoelectric Materials, Phenomena, and Applications: A Bird's Eye View. MRS Bulletin 31 (2006) März, S. 188-198

[33] Padture N. P.: Thermal Barrier Coatings for Gas-Turbine Engine Applications. Science 296 (2002) April, S. 280-284

[34] Kittel Charles, Fong C. Y.: Quantum Theory of Solids. 2nd rev. print. New York : Wiley 1987

[35] Goodson K. E.: Ordering Up the Minimum Thermal Conductivity of Solids. Science 315 (2007) Januar, S. 342

[36] Granta, CES Edupack: Material and Process Selection Charts. 2009

[37] Pan Wei, Phillpot Simon R., Wan Chunlei, Chernatynskiy Aleksandr, Qu Zhixue: Low Thermal Conductivity Oxides. MRS Bulletin 37 (2012) Oktober, S. 917-922

[38] Kim Woochul, Zide Joshua, Gossard Arthur, Klenov Dmitri, Stemmer Susanne, Shakouri Ali, Majumdar Arun: Thermal Conductivity Reduction and Thermoelectric Figure of Merit Increase by Embedding Nanoparticles in Crystalline Semiconductors. Physical Review Letters 96 (2006) Februar 
[39] Costescu R. M., Cahill D. G., Fabreguette F. H., Sechrist Z. A., George S. M.: Ultra-Low Thermal Conductivity in W/A2O3 Nanolaminates. Science 303 (2004) Februar, S. 989

[40] Maldovan Martin: Phonon Wave Interference and Thermal Bandgap Materials. Nature Materials 14 (2015) Juni, S. 667-674

[41] Venkatasubramanian Rama: Lattice Thermal Conductivity Reduction and Phonon Localizationlike Behavior in Superlattice Structures. Phys. Rev. B 61 (2000) Januar, S. 3091-3097

[42] Tamura Shin-ichiro, Tanaka Yukihiro, Maris Humphrey J.: Phonon Group Velocity and Thermal Conduction in Superlattices. Physical Review B 60 (1999) Juli, S. 2627-2630

[43] Balandin Alexander, Wang Kang L.: Significant Decrease of the Lattice Thermal Conductivity due to Phonon Confinement in a Free-Standing Semiconductor Quantum Well. Physical Review B 58 (1998) Juli, S. 1544-1549

[44] Regner Keith T., Sellan Daniel P., Su Zonghui, Amon Cristina H., McGaughey Alan J., Malen Jonathan A.: Broadband Phonon Mean Free Path Contributions to Thermal Conductivity Measured Using Frequency Domain Thermoreflectance. Nature Communications 4 (2013) März, S. 1640

[45] Tang G. H., Zhao Y., Zhai G. X., Bi C.: Phonon Boundary Scattering Effect on Thermal Conductivity of Thin Films. Journal of Applied Physics 110 (2011), S. 046102

[46] Vossen John L. (Hrsg.), Kern Werner (Hrsg.): Thin Film Processes II. Boston : Academic Press 1991

[47] Chrisey Douglas B. (Hrsg.), Hubler G. K. (Hrsg.): Pulsed Laser Deposition of Thin Films. New York: J. Wiley 1994

[48] Eason Robert (Hrsg.): Pulsed Laser Deposition of Thin Films: Applications-Led Growth of Functional Materials. Hoboken, N.J : Wiley-Interscience 2007

[49] Hublen Graham K.: Pulsed Laser Deposition. MRS Bulletin 17 (1992) Februar, S. 26-29

[50] Lowndes Douglas H., Geohegan D. B., Puretzky A. A., Norton D. P., Rouleau C. M.: Synthesis of Novel Thin-Film Materials by Pulsed Laser Deposition. Science 273 (1996) August, S. 898

[51] Krebs Hans-Ulrich, Weisheit Martin, Faupel Jörg, Süske Erik, Scharf Thorsten, Funse Christian, Störmer Michael, Sturm Kai, Seibt Michael, Kijewski Harald, Nelke Dorit, Panchenko Elena, Buback Michael: Pulsed Laser Deposition (PLD) A Versatile Thin Film Technique. In: Kramer Bernhard (Hrsg.): Advances in Solid State Physics. Berlin, Heidelberg : Springer Berlin Heidelberg 2003 S. 505-518 
[52] Krebs Hans-Ulrich, Bremert Olaf: Pulsed Laser Deposition of Thin Metallic Alloys. Applied Physics Letters 62 (1993), S. 2341

[53] Lunney James G.: Pulsed Laser Deposition of Metal and Metal Multilayer Films. Applied Surface Science 86 (1995) Februar, S. 79-85

[54] Oszwaldowski M., Berus T., Rzeszutek J., Sidorchuk P., Polit J., Sheregit E., Szade J.: Pulsed Laser Deposition of II-VI Semiconductor Thin Films and their Layered Structures. Journal of Alloys and Compounds 371 (2004) Mai, S. 164-167

[55] Dijkkamp D., Venkatesan T., Wu X. D., Shaheen S. A., Jisrawi N., Min-Lee Y. H., McLean W. L., Croft M.: Preparation of Y-Ba-Cu Oxide Superconductor Thin Films Using Pulsed Laser Evaporation from High Tc Bulk Material. Applied Physics Letters 51 (1987), S. 619

[56] Bubb Daniel M., Horwitz J. S., Callahan J. H., McGill R. A., Houser E. J., Chrisey D. B., Papantonakis M. R., Haglund R. F., Galicia M. C., Vertes A.: Resonant Infrared Pulsed-Laser Deposition of Polymer Films Using a Free-Electron Laser. Journal of Vacuum Science \& Technology A: Vacuum, Surfaces, and Films 19 (2001), S. 2698

[57] Schlenkrich Felix, Seyffarth Susanne, Fuchs Britta, Krebs Hans-Ulrich: Pulsed Laser Deposition of Polymer-metal Nanocomposites. Applied Surface Science 257 (2011) April, S. 5362-5365

[58] Tanaka Yukihiro, Tomoyasu Yoshinobu, Tamura Shin-ichiro: Band Structure of Acoustic Waves in Phononic Lattices: Two-Dimensional Composites with Large Acoustic Mismatch. Physical Review B 62 (2000) September, S. 7387-7392

[59] Peterson R.E., Anderson A.C.: Acoustic-Mismatch Model of the Kaptiza Resistance. Physics Letters A 40 (1972) Juli, S. 317-319

[60] Swartz E. T., Pohl R. O.: Thermal Resistance at Interfaces. Applied Physics Letters 51 (1987), S. 2200

[61] Grahn H.T., Maris H.J., Tauc J.: Picosecond Ultrasonics. IEEE Journal of Quantum Electronics 25 (Dec./1989), S. 2562-2569

[62] Sabbah A. J., Riffe D. M.: Femtosecond Pump-Probe Reflectivity Study of Silicon Carrier Dynamics. Physical Review B 66 (2002) Oktober

[63] Righini F., Bussolino G. C., Rosso A., Roberts R. B.: Thermal Conductivity by a Pulse-Heating Method: Theory and Experimental Apparatus. International Journal of Thermophysics 11 (1990) Juli, S. 629-641

[64] Cahill David G.: Thermal Conductivity Measurement from 30 to 750 K: The $3 \omega$ Method. Review of Scientific Instruments 61 (1990), S. 802 
[65] Jauregui J., Matthias E.: Thermal Diffusivities Measured by Photodisplacement Detection of Transient Thermal Gratings. Applied Physics A Solids and Surfaces 54 (1992) Januar, S. $35-39$

[66] Parker W. J., Jenkins R. J., Butler C. P., Аввотt G. L.: Flash Method of Determining Thermal Diffusivity, Heat Capacity, and Thermal Conductivity. Journal of Applied Physics 32 (1961), S. 1679

[67] Paddock Carolyn A., Eesley Gary L.: Transient Thermoreflectance from Thin Metal Films. Journal of Applied Physics 60 (1986), S. 285

[68] Paddock Carolyn A., Eesley Gary L.: Transient Thermoreflectance from Metal Films. Optics Letters 11 (1986) Mai, S. 273

[69] Touzelbaev M. N., Zhou P., Venkatasubramanian R., Goodson K. E.: Thermal Characterization of Bi[sub 2]Te[sub 3]/Sb[sub 2]Te[sub 3] Superlattices. Journal of Applied Physics 90 (2001), S. 763

[70] Andrew N. Smith, John L. Hostetler,: Thermal Boundary Resistance Measurements Using A Transient Thermoreflectance Technique. Microscale Thermophysical Engineering 4 (2000) Januar, S. 51-60

[71] Jagannadham K.: Effect of Interfacial Interactions on the Thermal Conductivity and Interfacial Thermal Conductance in Tungsten-graphene Layered Structure. Journal of Vacuum Science \& Technology A: Vacuum, Surfaces, and Films 32 (2014) September, S. 051101

[72] Panzer M. A., Zhang G., Mann D., Hu X., Pop E., Dai H., Goodson K. E.: Thermal Properties of Metal-Coated Vertically Aligned Single-Wall Nanotube Arrays. Journal of Heat Transfer 130 (2008), S. 052401

[73] Käding O. W., SKurk H., Goodson K. E.: Thermal Conduction in Metallized SiliconDioxide Layers on Silicon. Applied Physics Letters 65 (1994), S. 1629

[74] Carslaw H. S., Jaeger J. C.: Conduction of Heat in Solids. 2nd ed. Oxford [Oxfordshire] : New York : Clarendon Press ; Oxford University Press 1986

[75] Döring F., Eberl C., Schlenkrich S., Schlenkrich F., Hoffmann S., Liese T., Krebs H. U., Pisana S., Santos T., Schuhmann H., Seibt M., Mansurova M., Ulrichs H., Zbarsky V., MünZenberg M.: Phonon Localization in Ultrathin Layered Structures. Applied Physics A 119 (2015) April, S. 11-18 
[76] Döring Florian, Ulrichs Henning, Pagel Sinja, Müller Markus, Mansurova Maria, Müller Matthias, Eberl Christian, Erichsen Torben, Huebner Dennis, VanA Philipp, Mann Klaus, Münzenberg Markus, Krebs Hans-Ulrich: Confinement of Phonon Propagation in Laser Deposited Tungsten/Polycarbonate Multilayers. New Journal of Physics 18 (2016) September, S. 092002

[77] DöRING Florian, KRebs Hans-Ulrich: Thermal Conductivity Measurement by Transient Thermoreflectometry Using High-Fluence Excimer Laser Pulses at $248 \mathrm{Nm}$ Wavelength. High Temperatures-High Pressures Accepted August 11, 2016

[78] Döring Florian, Major Anna, Eberl Christian, Krebs Hans-Ulrich: Minimized Thermal Conductivity in Highly Stable Thermal Barrier W/ZrO2 Multilayers. Applied Physics A 122 (2016) Oktober

[79] Cheng K. C., FujII T.: Heat in History Isaac Newton and Heat Transfer. Heat Transfer Engineering 19 (1998) Januar, S. 9-21

[80] Narasimhan T. N.: Fourier's Heat Conduction Equation: History, Influence, and Connections. Reviews of Geophysics 37 (1999) Februar, S. 151-172

[81] Cahill David G., Ford Wayne K., Goodson Kenneth E., Mahan Gerald D., Majumdar Arun, Maris Humphrey J., Merlin Roberto, Phillpot Simon R.: Nanoscale Thermal Transport. Journal of Applied Physics 93 (2003), S. 793-818

[82] Ho C. Y., Powell R. W., Liley P. E.: Thermal Conductivity of the Elements: A Comprehensive Review. Journal of Physical and Chemical Reference Data 3 (1974)

[83] Ho C. Y., Powell R. W., Liley P. E.: Thermal Conductivity of the Elements. Journal of Physical and Chemical Reference Data 1 (1972), S. 279-421

[84] Lee S.-M., Cahill David G., Allen Thomas H.: Thermal Conductivity of Sputtered Oxide Films. Phys. Rev. B 52 (1995) Juli, S. 253-257

[85] Costescu Ruxandra M., Wall Marcel A., Cahill David G.: Thermal Conductance of Epitaxial Interfaces. Phys. Rev. B 67 (2003) Februar, S. 054302

[86] Swartz E. T., Pohl R. O.: Thermal Boundary Resistance. Rev. Mod. Phys. 61 (1989) Juli, S. 605-668

[87] Carey V. P., Chen G., Grigoropoulos C., Kaviany M., Majumdar A.: A Review of Heat Transfer Physics. Nanoscale and Microscale Thermophysical Engineering 12 (2008), S. 1-60

[88] E. Patrick, C. John: Introduction to Nanoscale Thermal Conduction. In: BeLmiloud Aziz (Hrsg.): Heat Transfer - Mathematical Modelling, Numerical Methods and Information Technology. InTech Februar 2011 
[89] Einstein A.: Die Plancksche Theorie der Strahlung und die Theorie der spezifischen Wärme. Annalen der Physik 327 (1906), S. 180-190

[90] Glassbrenner C. J., Slack Glen A.: Thermal Conductivity of Silicon and Germanium from 3ifmmode else $^{\circ}$ K to the Melting Point. Phys. Rev. 134 (1964) Mai, S. A1058-A1069

[91] Vineis Christopher J., Shakouri Ali, Majumdar Arun, Kanatzidis Mercouri G.: Nanostructured Thermoelectrics: Big Efficiency Gains from Small Features. Advanced Materials 22 (2010) September, S. 3970-3980

[92] Callaway Joseph: Model for Lattice Thermal Conductivity at Low Temperatures. Physical Review 113 (1959) Februar, S. 1046-1051

[93] PeIERls Rudolf: Zur Kinetischen Theorie Der Wärmeleitung in Kristallen. Annalen der Physik 395 (1929), S. 1055-1101

[94] Ziman J. M.: Electrons and Phonons: The Theory of Transport Phenomena in Solids. Oxford : New York : Clarendon Press ; Oxford University Press 2001 (Oxford classic texts in the physical sciences)

[95] Ren Shang Y., Dow John D.: Thermal Conductivity of Superlattices. Physical Review B 25 (1982) März, S. 3750-3755

[96] Casimir HBG: Note on the Conduction of Heat in Crystals. Physica 5 (1938), S. 495-500

[97] Debye P.: Zur Theorie der spezifischen Wärmen. Annalen der Physik 344 (1912), S. 789-839

[98] Klemens P. G., Flügge S. (Hrsg.): Handbuch Der Physik. Bd. 14. Berlin : SpringerVerlag 1956

[99] Klemens P. G.: Solid State Physics. New York: Academic Press Inc. 1958 (7)

[100] Slack Glen A.: Thermal Conductivity of $\mathrm{MgO}, \mathrm{Al} 2 \mathrm{O} 3$, Mg Al $2 \mathrm{O} 4$, and Fe $3 \mathrm{O} 4$ Crystals from $3^{\circ}$ to $300^{\circ} \mathrm{K}$. Physical Review 126 (1962) April, S. 427-441

[101] Slack Glen A., Galginaitis S.: Thermal Conductivity and Phonon Scattering by Magnetic Impurities in CdTe. Physical Review 133 (1964) Januar, S. A253-A268

[102] Zou Jie, Balandin Alexander: Phonon Heat Conduction in a Semiconductor Nanowire. Journal of Applied Physics 89 (2001), S. 2932

[103] Simkin M.V., Mahan G.D.: Umklapp Scattering and Heat Conductivity of Superlattices. In: Symposium Z -Thermoelectric Materials 2000 - The Next Generation Materials for Small-Scale Refrigeration and Power Generation Applications Bd. 6262000 (MRS Proceedings) S. Z9.5 (2 pages) 
[104] Ratsifaritana C. A., Klemens P. G.: Scattering of Phonons by Vacancies. International Journal of Thermophysics 8 (1987), S. 737-750

[105] Klemens P. G.: The Scattering of Low-Frequency Lattice Waves by Static Imperfections. Proceedings of the Physical Society. Section A 68 (1955), S. 1113

[106] Rowe D. M., Shukla V. S., Savvides N.: Phonon Scattering at Grain Boundaries in Heavily Doped Fine-Grained Silicon-Germanium Alloys. Nature 290 (1981) April, S. 765766

[107] Schelling P. K., Phillpot S. R., Keblinski P.: Kapitza Conductance and Phonon Scattering at Grain Boundaries by Simulation. Journal of Applied Physics 95 (2004), S. 6082-6091

[108] Cahill David, Bullen Andrew, Lee Seung-Min: Interface Thermal Conductance and the Thermal Conductivity of Multilayer Thin Films. High Temperatures-High Pressures 32 (2000), S. 135-142

[109] Hicks L. D., Dresselhaus M. S.: Effect of Quantum-Well Structures on the Thermoelectric Figure of Merit. Phys. Rev. B 47 (1993) Mai, S. 12727-12731

[110] Tien Chang L. (Hrsg.), Majumdar Arunava (Hrsg.), Gerner F. M. (Hrsg.): Microscale Energy Transport. Washington, D.C : Taylor \& Francis 1998 (Series in chemical and mechanical engineering)

[111] Johnson Jeremy A., Maznev A. A., Cuffe John, Eliason Jeffrey K., Minnich Austin J., Kehoe Timothy, Torres Clivia M. S., Chen Gang, Nelson Keith A.: Direct Measurement of Room-Temperature Nondiffusive Thermal Transport Over Micron Distances in a Silicon Membrane. Phys. Rev. Lett. 110 (2013) Januar, S. 025901

[112] Esaki Leo, Tsu Ray: Superlattice and Negative Differential Conductivity in Semiconductors. I B M J RES DEVELOP 14 (1970), S. 61-65

[113] Schuller Ivan K.: New Class of Layered Materials. Physical Review Letters 44 (1980) Juni, S. 1597-1600

[114] Parkin S. S. P., More N., Roche K. P.: Oscillations in Exchange Coupling and Magnetoresistance in Metallic Superlattice Structures: Co/Ru, Co/Cr, and $\mathrm{Fe} / \mathrm{Cr}$. Physical Review Letters 64 (1990) Mai, S. 2304-2307

[115] Andres Ronald P., Bielfeld Jeffrey D., Henderson Jason I., Janes David B., others: Self-Assembly of a Two-Dimensional Superlattice of Molecularly Linked Metal Clusters. Science 273 (1996), S. 1690 
[116] Osterhoff Markus, Eberl Christian, Döring Florian, Wilke Robin N., Wallentin Jesper, Krebs Hans-Ulrich, Sprung Michael, SAlditt Tim: Towards Multi-Order Hard $X$-Ray Imaging with Multilayer Zone Plates. Journal of Applied Crystallography 48 (2015) Februar, S. 116-124

[117] Chen G., Tien C. L., Wu X., Smith J. S.: Thermal Diffusivity Measurement of GaAs/AlGaAs Thin-Film Structures. Journal of Heat Transfer 116 (1994) Mai, S. 325-331

[118] Waschke Christian, Roskos Hartmut G., Schwedler Ralf, Leo Karl, Kurz Heinrich, KöHLER Klaus: Coherent Submillimeter-Wave Emission from Bloch Oscillations in a Semiconductor Superlattice. Physical Review Letters 70 (1993) Mai, S. 3319-3322

[119] Collier C. P., Vossmeyer T., Heath J. R.: Nanocrystal Superlattices. Annual Review of Physical Chemistry 49 (1998) Oktober, S. 371-404

[120] Liang Bin, Yuan Bo, Cheng Jian-chun: Acoustic Diode: Rectification of Acoustic Energy Flux in One-Dimensional Systems. Physical Review Letters 103 (2009) September

[121] Lu Yan-Qing, Wan Zhi-Liang, Wang Quan, XI Yuan-Xin, Ming Nai-Ben: ElectroOptic Effect of Periodically Poled Optical Superlattice LiNbO[sub 3] and Its Applications. Applied Physics Letters 77 (2000), S. 3719

[122] Wei Y., Gin A., Razeghi M., Brown G. J.: Advanced InAs/GaSb Superlattice Photovoltaic Detectors for Very Long Wavelength Infrared Applications. Applied Physics Letters 80 (2002), S. 3262

[123] Capasso Federico: Band-Gap Engineering via Graded Gap, Superlattice, and Periodic Doping Structures: Applications to Novel Photodetectors and Other Devices. Journal of Vacuum Science \& Technology B: Microelectronics and Nanometer Structures 1 (1983), S. 457

[124] Faist Jerome, Capasso Federico, Sivco Deborah L., Sirtori Carlo, Hutchinson Albert L., Chо Alfred Y., отнеRs: Quantum Cascade Laser. Science 264 (1994), S. 553-556

[125] Whitlow L. W., Hirano T.: Superlattice Applications to Thermoelectricity. Journal of Applied Physics 78 (1995), S. 5460

[126] Chowdhury Ihtesham, Prasher Ravi, Lofgreen Kelly, Chrysler Gregory, Narasimhan Sridhar, Mahajan Ravi, Koester David, Alley Randall, VenkataSUbRAMANIAN Rama: On-Chip Cooling by Superlattice-Based Thin-Film Thermoelectrics. Nature Nanotechnology 4 (2009) April, S. 235-238 
[127] Venkatasubramanian R., Colpitts T., Watko E., Lamvik M., El-Masry N.: MOCVD of Bi2Te3, Sb2Te3 and their Superlattice Structures for Thin-Film Thermoelectric Applications. Journal of Crystal Growth 170 (1997) Januar, S. 817-821

[128] Nolas G. S., Morelli D. T., Tritt Terry M.: SKUTTERUDITES: A Phonon-GlassElectron Crystal Approach to Advanced Thermoelectric Energy Conversion Applications. Annual Review of Materials Science 29 (1999) August, S. 89-116

[129] VININg Cronin B.: An Inconvenient Truth about Thermoelectrics. Nature Materials 8 (2009) Februar, S. 83-85

[130] Franz R., Wiedemann G.: Ueber die Wärme-Leitungsfähigkeit der Metalle. Annalen der Physik und Chemie 165 (1853), S. 497-531

[131] Hicks L. D., Harman T. C., Sun X., Dresselhaus M. S.: Experimental Study of the Effect of Quantum-Well Structures on the Thermoelectric Figure of Merit. Physical Review B 53 (1996) April, S. R10493-R10496

[132] Hicks L. D., Dresselhaus M. S.: Use of Quantum-Well Superlattices to Obtain a High Figure of Merit from Nonconventional Thermoelectric Materials. MRS Proceedings 326 (1993) Januar

[133] Wright D. A.: Thermoelectric Properties of Bismuth Telluride and Its Alloys. Nature 181 (1958) März, S. 834-834

[134] Yao Takafumi: Thermal Properties of AlAs/GaAs Superlattices. Applied Physics Letters 51 (1987), S. 1798

[135] Capinski W. S., Maris H. J., Ruf T., Cardona M., Ploog K., Katzer D. S.: ThermalConductivity Measurements of GaAs/AlAs Superlattices Using a Picosecond Optical Pumpand-Probe Technique. Physical Review B 59 (1999) März, S. 8105-8113

[136] Lee S.-M., Cahill David G., Venkatasubramanian Rama: Thermal Conductivity of Si-Ge Superlattices. Applied Physics Letters 70 (1997), S. 2957

[137] Borca-Tasciuc Theodorian, Liu Weili, Liu Jianlin, Zeng Taofang, Song David W., Moore Caroline D., Chen Gang, Wang Kang L., Goorsky Mark S., Radetic Tamara, OTHERs: Thermal Conductivity of Symmetrically Strained Si/Ge Superlattices. Superlattices and microstructures 28 (2000), S. 199-206

[138] Yu X. Y., Chen G., Verma A., Smith J. S.: Temperature Dependence of Thermophysical Properties of GaAs/AlAs Periodic Structure. Applied Physics Letters 67 (1995), S. 3554 
[139] Huxtable Scott T., Abramson Alexis R., Tien Chang-Lin, Majumdar Arun, LaBounty Chris, Fan Xiaofeng, Zeng Gehong, Bowers John E., ShaKouri Ali, Croкe Edward T.: Thermal Conductivity of Si/SiGe and SiGe/SiGe Superlattices. Applied Physics Letters 80 (2002), S. 1737

[140] S.-M. Lee George Matamis David G. C: Thin-Film Materials And Minimum Thermal Conductivity. Microscale Thermophysical Engineering 2 (1998) Februar, S. 31-36

[141] Ju Y. S., Hung M.-T., Carey M. J., Cyrille M.-C., Childress J. R.: Nanoscale Heat Conduction across Tunnel Junctions. Applied Physics Letters 86 (2005), S. 203113

[142] Chiritescu Catalin, Cahill David G., Nguyen Ngoc, Johnson David, Bodapati Arun, Keblinski Pawel, Zschack Paul: Ultralow Thermal Conductivity in Disordered, Layered WSe $e_{2}$ Crystals. Science 315 (2007) Januar, S. 351

[143] Lee S.-M., Cahill David G.: Heat Transport in Thin Dielectric Films. Journal of Applied Physics 81 (1997), S. 2590

[144] Eberl Christian, Liese Tobias, Schlenkrich Felix, Döring Florian, Hofsäss Hans, KRebs Hans-Ulrich: Enhanced Resputtering and Asymmetric Interface Mixing in W/Si Multilayers. Applied Physics A 111 (2013) Mai, S. 431-437

[145] Fähler Sebastian, Krebs Hans-Ulrich: Calculations and Experiments of Material Removal and Kinetic Energy during Pulsed Laser Ablation of Metals. Applied Surface Science 96-98 (1996) April, S. 61-65

[146] Krebs H-U: Characteristic Properties of Laser-Deposited Metallic Systems. International Journal of Non-Equilibrium Processing(UK) 10 (1997), S. 3-24

[147] Floro J. A., Hearne S. J., Hunter J. A., Kotula P., Chason E., Seel S. C., Thompson C. V.: The Dynamic Competition between Stress Generation and Relaxation Mechanisms during Coalescence of Volmer-Weber Thin Films. Journal of Applied Physics 89 (2001), S. 4886

[148] Simkin MV, Mahan GD: Minimum Thermal Conductivity of Superlattices. Physical Review Letters 84 (2000), S. 927

[149] Nath Prem, Chopra K.L.: Thermal Conductivity of Copper Films. Thin Solid Films 20 (1974) Januar, S. 53-62

[150] Budd H., Vannimenus J.: Thermal Boundary Resistance. Phys. Rev. Lett. 26 (1971) Juni, S. $1637-1640$

[151] Kapitza P. L.: Zh. Eksp. Teor. Fiz 11 (1941)

[152] Khalatnikov I. M.: Teploobmen Mezhdu Tverdym Telom I Geliem II. Sov. Phys. JETP 22 (1952), S. 687-704 
[153] Young D. A., Maris H. J.: Lattice-Dynamical Calculation of the Kapitza Resistance between Fcc Lattices. Phys. Rev. B 40 (1989) August, S. 3685-3693

[154] Pettersson Sune, Mahan G. D.: Theory of the Thermal Boundary Resistance between Dissimilar Lattices. Phys. Rev. B 42 (1990) Oktober, S. 7386-7390

[155] Persson B. N. J., Ueba H.: Heat Transfer between Graphene and Amorphous SiO 2. Journal of Physics: Condensed Matter 22 (2010), S. 462201

[156] Stoner R. J., Maris H. J.: Kapitza Conductance and Heat Flow between Solids at Temperatures from 50 to 300 K. Phys. Rev. B 48 (1993) Dezember, S. 16373-16387

[157] Hyldgaard Per, Mahan G. D.: Phonon Superlattice Transport. Phys. Rev. B 56 (1997) November, S. 10754-10757

[158] Cahill David G., Braun Paul V., Chen Gang, Clarke David R., Fan Shanhui, Goodson Kenneth E., Keblinski Pawel, King William P., Mahan Gerald D., Majumdar Arun, Maris Humphrey J., Phillpot Simon R., Pop Eric, Shi Li: Nanoscale Thermal Transport. II. 2003-2012. Applied Physics Reviews 1 (2014) März, S. 011305

[159] ŻAK D., NAKWASKi W.: Thermal Resistance of GaAs/AlAs Superlattices Used in Modern Light-Emitting Diodes. Opto-Electronics Review 22 (2014) Januar

[160] Hahn Konstanze R., Puligheddu Marcello, Colombo Luciano: Thermal Boundary Resistance at Si/Ge Interfaces Determined by Approach-to-Equilibrium Molecular Dynamics Simulations. Physical Review B 91 (2015) Mai

[161] Narayanamurti V., Störmer H. L., Chin M. A., Gossard A. C., Wiegmann W.: Selective Transmission of High-Frequency Phonons by a Superlattice: The "Dielectric" Phonon Filter. Physical Review Letters 43 (1979) Dezember, S. 2012-2016

[162] Barker A. S., Merz J. L., Gossard A. C.: Study of Zone-Folding Effects on Phonons in Alternating Monolayers of GaAs-AlAs. Physical Review B 17 (1978) April, S. 3181-3196

[163] Ravichandran Jayakanth, Yadav Ajay K., Cheaito Ramez, Rossen Pim B., Soukiassian Arsen, Suresha S. J., Duda John C., Foley Brian M., Lee Che-Hui, Zhu Ye, Lichtenberger Arthur W., Moore Joel E., Muller David A., Schlom Darrell G., Hopkins Patrick E., Majumdar Arun, Ramesh Ramamoorthy, Zurbuchen Mark A.: Crossover from Incoherent to Coherent Phonon Scattering in Epitaxial Oxide Superlattices. Nature Materials 13 (2013) Dezember, S. 168-172

[164] Wang Yan, Huang Haoxiang, Ruan Xiulin: Decomposition of Coherent and Incoherent Phonon Conduction in Superlattices and Random Multilayers. Physical Review B 90 (2014) Oktober 
[165] Wang Yan, Gu Chongjie, Ruan Xiulin: Optimization of the Random Multilayer Structure to Break the Random-Alloy Limit of Thermal Conductivity. Applied Physics Letters 106 (2015) Februar, S. 073104

[166] Maldovan Martin: Narrow Low-Frequency Spectrum and Heat Management by Thermocrystals. Physical Review Letters 110 (2013) Januar

[167] Wu Z. L., Reichling M., Hu X.-Q., Balasubramanian K., Guenther K. H.: Absorption and Thermal Conductivity of Oxide Thin Films Measured by Photothermal Displacement and Reflectance Methods. Applied Optics 32 (1993) Oktober, S. 5660

[168] Cahill David G.: Analysis of Heat Flow in Layered Structures for Time-Domain Thermoreflectance. Review of Scientific Instruments 75 (2004) Dezember, S. 5119-5122

[169] Кон Yee K., Singer Suzanne L., Kim Woochul, Zide Joshua M. O., Lu Hong, Cahill David G., Majumdar Arun, Gossard Arthur C.: Comparison of the $3 \omega$ Method and Time-Domain Thermoreflectance for Measurements of the Cross-Plane Thermal Conductivity of Epitaxial Semiconductors. Journal of Applied Physics 105 (2009), S. 054303

[170] Burzo M.G., Komarov P.L., RaAd P.E.: Optimized Thermo-Reflectance System for Measuring the Thermal Properties of Thin-Films and their Interfaces IEEE 2006 S. 87-94

[171] Komarov Pavel L., Burzo Mihai G., Kaytaz Gunhan, RaAd Peter E.: Transient Thermo-Reflectance Measurements of the Thermal Conductivity and Interface Resistance of Metallized Natural and Isotopically-Pure Silicon. Microelectronics Journal 34 (2003) Dezember, S. 1115-1118

[172] Miyake Syugo, Kita Takashi, Miyake Aya, Ikeda Ken-ichi, Takamatsu Hiroyuki: Analysis of Thermoreflectance Signals and Characterization of Thermal Conductivity of Metal Thin Films. Review of Scientific Instruments 80 (2009), S. 124901

[173] Figure Created by Dr. Henning Ulrichs.

[174] Bechtel J. H.: Heating of Solid Targets with Laser Pulses. Journal of Applied Physics 46 (1975), S. 1585

[175] Wolfe R.: The Theory of the Reflectivity of Metals. Proceedings of the Physical Society. Section A 68 (1955), S. 121

[176] Zhang ChungWei, Bi KeDong, Wang JianLi, Ni ZhongHua, Chen YunFei: Measurement of Thermal Boundary Conductance between Metal and Dielectric Materials Using Femtosecond Laser Transient Thermoreflectance Technique. Science China Technological Sciences 55 (2012) April, S. 1044-1049

[177] BeER: Bestimmung der Absorption des rothen Lichts in farbigen Flüssigkeiten. Annalen der Physik und Chemie 162 (1852), S. 78-88 
[178] Farzaneh M., Maize K., Lüerssen D., Summers J. A., Mayer P. M., Raad P. E., Pipe K. P., Shakouri A., Ram R. J., Hudgings Janice A.: CCD-Based Thermoreflectance Microscopy: Principles and Applications. Journal of Physics D: Applied Physics 42 (2009), S. 143001

[179] Willardson R. K., Beer Albert C.: Infrared Detectors. 2: [...]. New York, NY : Acad. Press 1977 (Semiconductors and semimetals). - OCLC: 58621086

[180] Guidotti Daniel, Wilman John G.: Novel and Nonintrusive Optical Thermometer. Applied Physics Letters 60 (1992), S. 524

[181] Wang Yuxin, Park Ji Y., Koh Yee K., Cahill David G.: Thermoreflectance of Metal Transducers for Time-Domain Thermoreflectance. Journal of Applied Physics 108 (2010), S. 043507

[182] Cielo P.: Pulsed Photothermal Evaluation of Layered Materials. Journal of Applied Physics 56 (1984), S. 230

[183] Balageas D. L., Krapez J. C., Cielo P.: Pulsed Photothermal Modeling of Layered Materials. Journal of Applied Physics 59 (1986), S. 348

[184] Degiovanni Alain: Diffusivité et Méthode Flash. Revue générale de thermique 185 (1977), S. 420-442

[185] Prokнorov A. M. (Hrsg.): Laser Heating of Metals. Bristol ; Philadelphia : A. Hilger 1990 (The Adam Hilger series on optics and optoelectronics)

[186] Luckyanova M. N., Garg J., Esfarjani K., Jandl A., Bulsara M. T., Schmidt A. J., Minnich A. J., Chen S., Dresselhaus M. S., Ren Z., Fitzgerald E. A., Chen G.: Coherent Phonon Heat Conduction in Superlattices. Science 338 (2012) November, S. 936-939

[187] Gilmore David G. (Hrsg.): Spacecraft Thermal Control Handbook. 2nd ed. El Segundo, Calif : Aerospace Press 2002

[188] Limarga Andi M., Clarke David R.: Characterization of Electron Beam Physical Vapor-Deposited Thermal Barrier Coatings Using Diffuse Optical Reflectance. International Journal of Applied Ceramic Technology 6 (2009) Mai, S. 400-409

[189] Josell D., Cezairliyan A., Bonevich J. E.: Thermal Diffusion Through Multilayer Coatings: Theory and Experiment. International Journal of Thermophysics 19 (1998), S. 525-535

[190] Thomsen C., Strait J., Vardeny Z., Maris H. J., Tauc J., Hauser J. J.: Coherent Phonon Generation and Detection by Picosecond Light Pulses. Physical Review Letters 53 (1984) September, S. 989-992 
[191] MerLIN R.: Generating Coherent THz Phonons with Light Pulses. Solid State Communications 102 (1997) April, S. 207-220

[192] Schoenlein R. W., Lin W. Z., Fujimoto J. G., Eesley G. L.: Femtosecond Studies of Nonequilibrium Electronic Processes in Metals. Physical Review Letters 58 (1987) April, S. $1680-1683$

[193] Brorson S. D., Kazeroonian A., Moodera J. S., Face D. W., Cheng T. K., Ippen E. P., Dresselhaus M. S., Dresselhaus G.: Femtosecond Room-Temperature Measurement of the Electron-Phonon Coupling Constant $\gamma$ in Metallic Superconductors. Physical Review Letters 64 (1990) April, S. 2172-2175

[194] Ezzahri Y., Grauby S., Rampnoux J. M., Michel H., Pernot G., Claeys W., Dilhaire S., Rossignol C., Zeng G., Shakouri A.: Coherent Phonons in Si / Si Ge Superlattices. Physical Review B 75 (2007) Mai

[195] Djafari-Rouhani B., Dobrzynski L., Duparc O. H., Camley R. E., Maradudin A. A.: Sagittal Elastic Waves in Infinite and Semi-Infinite Superlattices. Physical Review B 28 (1983) August, S. 1711-1720

[196] Windt David L.: IMD—Software for Modeling the Optical Properties of Multilayer Films. Computers in Physics 12 (1998), S. 360

[197] Eberl Christian, Döring Florian, Liese Tobias, Schlenkrich Felix, Roos Burkhard, Hahn Matthias, Hoinkes Thomas, Rauschenbeutel Arno, Osterhoff Markus, Salditt Tim, Krebs Hans-Ulrich: Fabrication of Laser Deposited High-Quality Multilayer Zone Plates for Hard X-Ray Nanofocusing. Applied Surface Science 307 (2014) Juli, S. 638-644

[198] RöDER Johanna, LIESE Tobias, KRebs Hans-Ulrich: Material-Dependent Smoothing of Periodic Rippled Structures by Pulsed Laser Deposition. Journal of Applied Physics 107 (2010), S. 103515

[199] Döring F., Robisch A.L., Eberl C., Osterhoff M., Ruhlandt A., Liese T., Schlenkrich F., Hoffmann S., Bartels M., Salditt T., Krebs H.U.: Sub-5 Nm Hard X-Ray Point Focusing by a Combined Kirkpatrick-Baez Mirror and Multilayer Zone Plate. Optics Express 21 (2013) August, S. 19311

[200] Elsayed-Ali H. E., Norris T. B., Pessot M. A., Mourou G. A.: Time-Resolved Observation of Electron-Phonon Relaxation in Copper. Physical Review Letters 58 (1987) März, S. 1212-1215

[201] Taflove Allen (Hrsg.): Advances in Computational Electrodynamics: The FiniteDifference Time-Domain Method. Boston: Artech House 1998 
[202] Van Slyke S. A., Chen C. H., Tang C. W.: Organic Electroluminescent Devices with Improved Stability. Applied Physics Letters 69 (1996), S. 2160

[203] Mittal K. L. (Hrsg.): Metallized Plastics: Fundamentals and Applications. New York : Marcel Dekker 1998 (Plastics engineering)

[204] Toda Minoru, Thompson Mitchell: Novel Multi-Layer Polymer-Metal Structures for Use in Ultrasonic Transducer Impedance Matching and Backing Absorber Applications. IEEE Transactions on Ultrasonics, Ferroelectrics and Frequency Control 57 (2010) Dezember, S. 2818-2827

[205] Toda Minoru, Thompson Mitch: Detailed Investigations of Polymer/Metal Multilayer Matching Layer and Backing Absorber Structures for Wideband Ultrasonic Transducers. IEEE Transactions on Ultrasonics, Ferroelectrics, and Frequency Control 59 (2012) Februar, S. 231-242

[206] Affinito John, Martin Peter, Gross Mark, Coronado Chris, Greenwell Eric: Vacuum Deposited Polymer/Metal Multilayer Films for Optical Application. Thin Solid Films 270 (1995) Dezember, S. 43-48

[207] Tsumura A., Koezuka H., Ando T.: Macromolecular Electronic Device: Field-Effect Transistor with a Polythiophene Thin Film. Applied Physics Letters 49 (1986), S. 1210

[208] Koezuka H., Tsumura A.: Field-Effect Transistor Utilizing Conducting Polymers. Synthetic Metals 28 (1989) Januar, S. 753-760

[209] Prasher Ravi: Acoustic Mismatch Model for Thermal Contact Resistance of van Der Waals Contacts. Applied Physics Letters 94 (2009), S. 041905

[210] O'Connell Paul A., Wang Jian, Ishola T. A., McKenna Gregory B.: Exceptional Property Changes in Ultrathin Films of Polycarbonate: Glass Temperature, Rubbery Stiffening, and Flow. Macromolecules 45 (2012) März, S. 2453-2459

[211] Lassner Erik, Schubert Wolf-Dieter: Tungsten: Properties, Chemistry, Technology of the Element, Alloys and Chemical Compounds. New York : Springer 1999. OCLC: 466157044

[212] Deymier Pierre A. (Hrsg.): Acoustic Metamaterials and Phononic Crystals. Berlin ; New York : Springer 2013 (Springer series in solid-state sciences). - OCLC: ocn827925764

[213] Niemiec J M., Schultheisz C R., Schutte C, McKenna G B.: SPE ANTEC Tech. Pap. 41 (1995), 2402-2408 S.

[214] Osswald Tim A., Hernández-Ortiz Juan P.: Polymer Processing: Modeling and Simulation. Munich ; Cincinnati : Hanser Publishers 2006. - OCLC: ocm64288947 
[215] Peth Christian, Barkusky Frank, Mann Klaus: Near-Edge X-Ray Absorption Fine Structure Measurements Using a Laboratory-Scale XUV Source. Journal of Physics D: Applied Physics 41 (2008) Mai, S. 105202

[216] Majumdar Arun, Reddy Pramod: Role of Electron-phonon Coupling in Thermal Conductance of Metal-nonmetal Interfaces. Applied Physics Letters 84 (2004), S. 4768

[217] Hickson R.I., Barry S.I., Mercer G.N., Sidhu H.S.: Finite Difference Schemes for Multilayer Diffusion. Mathematical and Computer Modelling 54 (2011) Juli, S. 210220

[218] Perrin Bernard: Investigation of Short-Time Heat Transfer Effects by an Optical Pump-Probe Method. In: Volz Sebastian (Hrsg.): Microscale and Nanoscale Heat Transfer Bd. 107. Springer Berlin Heidelberg 2006 S. 333-359

[219] Little W. A.: The Transport Of Heat Between Dissimilar Solids At Low Temperatures. Canadian Journal of Physics 37 (1959) März, S. 334-349

[220] Chrisey D. B., Piqué A., McGill R. A., Horwitz J. S., Ringeisen B. R., Bubb D. M., Wu P. K.: Laser Deposition of Polymer and Biomaterial Films. Chemical Reviews 103 (2003) Februar, S. 553-576

[221] Dhez O, Ade H, Urquhart S.G: Calibrated NEXAFS Spectra of Some Common Polymers. Journal of Electron Spectroscopy and Related Phenomena 128 (2003) Januar, S. 85-96

[222] Fuchs Britta, Schlenkrich Felix, Seyffarth Susanne, Meschede Andreas, Rotzoll Robert, Vana Philipp, Grossmann Peter, Mann Klaus, Krebs Hans-Ulrich: Hardening of Smooth Pulsed Laser Deposited PMMA Films by Heating. Applied Physics A 98 (2010) März, S. 711-715

[223] Novikov V. N., Soкоlov A. P.: Poisson's Ratio and the Fragility of Glass-Forming Liquids. Nature 431 (2004) Oktober, S. 961-963

[224] Ulrichs Henning, Meyer Dennis, Müller Markus, Mansurova Maria, Döring Florian: Numerical Calculation of Laser-Induced Thermal Diffusion and Elastic Dynamics 2016 S. 040004

[225] Guo Yunlong, Morozov Anatoli, Schneider Dirk, Chung Jae W., Zhang Chuan, Waldmann Maike, Yao Nan, Fytas George, Arnold Craig B., Priestley Rodney D.: Ultrastable Nanostructured Polymer Glasses. Nature Materials 11 (2012) Februar, S. 337343

[226] Weishaupt K., Pietralla M.: The Elastic Behaviour of Polycarbonate in the Glassy State Determined by Brillouin Scattering. Journal of Materials Science 30 (1995), S. 5457-5460 
[227] Lippert Thomas: Laser Application of Polymers. In: Lippert Thomas. K. (Hrsg.): Polymers and Light Bd. 168. Berlin, Heidelberg : Springer Berlin Heidelberg 2004 S. 51-246

[228] Schneider Dirk, Liaqat Faroha, El Boudouti El H., El Hassouani Youssef, Djafari-Rouhani Bahram, Tremel Wolfgang, Butt Hans-Jürgen, Fytas George: Engineering the Hypersonic Phononic Band Gap of Hybrid Bragg Stacks. Nano Letters 12 (2012) Juni, S. 3101-3108

[229] Alonso-Redondo Elena, Huesmann Hannah, El Boudouti El-Houssaine, Tremel Wolfgang, Djafari-Rouhani Bahram, Butt Hans-Juergen, Fytas George: Phoxonic Hybrid Superlattice. ACS Applied Materials \& Interfaces 7 (2015) Juni, S. 12488-12495

[230] Gomopoulos N., Maschke D., Кон C. Y., Thomas E. L., Tremel W., Butt H.-J., Fytas G.: One-Dimensional Hypersonic Phononic Crystals. Nano Letters 10 (2010) März, S. $980-984$

[231] Saini G., Pezeril T., Torchinsky D.H., Yoon J., Kooi S.E., Thomas E.L., Nelson K.A.: Pulsed Laser Characterization of Multicomponent Polymer Acoustic and Mechanical Properties in the Sub-GHz Regime. Journal of Materials Research 22 (2007) März, S. 719-723

[232] Schneider Dirk, Liaqat Faroha, El Boudouti El H., El Abouti Ossama, Tremel Wolfgang, Butt Hans-Jürgen, DJafari-Rouhani Bahram, Fytas George: DefectControlled Hypersound Propagation in Hybrid Superlattices. Physical Review Letters 111 (2013) Oktober

[233] Walker P. M., Sharp J. S., Akimov A. V., Kent A. J.: Coherent Elastic Waves in a One-Dimensional Polymer Hypersonic Crystal. Applied Physics Letters 97 (2010), S. 073106

[234] Rytov SaM: Acoustical Properties of a Thinly Laminated Medium. Soviet Physics Acoustics 2 (1956), S. 68-80

[235] Matsuda Osamu, Larciprete Maria C., Li Voti Roberto, Wright Oliver B.: Fundamentals of Picosecond Laser Ultrasonics. Ultrasonics 56 (2015) Februar, S. 3-20

[236] Pietrak Karol, Wiśniewski Tomasz S.: Methods for Experimental Determination of Solid-Solid Interfacial Thermal Resistance with Application to Composite Materials. J. Power Technologies 94 (2014), S. 270-285

[237] Norris Pamela M., Caffrey Andrew P., Stevens Robert J., Klopf J. M., McLeskey James T., Sмiтн Andrew N.: Femtosecond Pump-probe Nondestructive Examination of Materials (Invited). Review of Scientific Instruments 74 (2003), S. 400 
[238] J. L. Hostetler, A. N. Smith, And P: Thin-Film Thermal Conductivity And Thickness Measurements Using Picosecond Ultrasonics. Microscale Thermophysical Engineering 1 (1997) Juli, S. 237-244

[239] Schmidt Aaron J., Chen Xiaoyuan, Chen Gang: Pulse Accumulation, Radial Heat Conduction, and Anisotropic Thermal Conductivity in Pump-Probe Transient Thermoreflectance. Review of Scientific Instruments 79 (2008), S. 114902

[240] Ujihara Kikuo: Reflectivity of Metals at High Temperatures. Journal of Applied Physics 43 (1972), S. 2376-2383

[241] Clemens Bruce M., Eesley Gary L., Paddock Carolyn A.: Time-Resolved Thermal Transport in Compositionally Modulated Metal Films. Physical Review B 37 (1988) Januar, S. $1085-1096$

[242] Gengler Jamie J., Roy Sukesh, Jones John G., Gord James R.: Two-Color Time-Domain Thermoreflectance of Various Metal Transducers with an Optical Parametric Oscillator. Measurement Science and Technology 23 (2012) Mai, S. 055205

[243] van Der Tempel L.: Thermal Conductivity of a Glass: II. The Empirical Model. Glass Physics and Chemistry 28 (2002), S. 147-152

[244] Schlichting KW, Padture NP, Klemens PG: Thermal Conductivity of Dense and Porous Yttria-Stabilized Zirconia. Journal of materials science 36 (2001), S. 3003-3010

[245] Oh Dong-Wook, Ravichandran Jayakanth, Liang Chen-Wei, Siemons Wolter, Jalan Bharat, Brooks Charles M., Huijben Mark, Schlom Darrell G., Stemmer Susanne, Martin Lane W., Majumdar Arun, Ramesh Ramamoorthy, Cahill David G.: Thermal Conductivity as a Metric for the Crystalline Quality of SrTiOlsub 3] Epitaxial Layers. Applied Physics Letters 98 (2011), S. 221904

[246] Slifka A. J., Filla B. J., Phelps J.M.: Thermal Conductivity of Magnesium Oxide from Absolute, Steady-State Measurements. J. Res. Natl. Inst. Stand. Technol. 103 (1998), S. 357-363

[247] B. L. WANG, J. C. Han, S. Y. Du: Crack Problems For Functionally Graded Materials Under Transient Thermal Loading. Journal of Thermal Stresses 23 (2000) März, S. 143-168

[248] Volz Sebastian, Ordonez-Miranda Jose, Shchepetov Andrey, Prunnila Mika, Ahopelto Jouni, Pezeril Thomas, Vaudel Gwenaelle, Gusev Vitaly, Ruello Pascal, Weig Eva M., Schubert Martin, Hettich Mike, Grossman Martin, Dekorsy Thomas, Alzina Francesc, Graczykowski Bartlomiej, Chavez-Angel Emigdio, Sebastian Reparaz J., Wagner Markus R., Sotomayor-Torres Clivia M., Bock Wurst, Xiong Shiyun, Neogi Sanghamitra, Donadio Davide: Nanophononics: State of the Art and Perspectives. The European Physical Journal B 89 (2016) Januar 
[249] Sсотт H. G.: Phase Relationships in the Zirconia-Yttria System. Journal of Materials Science 10 (1975) September, S. 1527-1535

[250] Kurlov A. S., Gusev A. I.: Neutron and X-Ray Diffraction Study and Symmetry Analysis of Phase Transformations in Lower Tungsten Carbide W 2 C. Physical Review B 76 (2007) November

[251] Cao X.Q., Vassen R., Stoever D.: Ceramic Materials for Thermal Barrier Coatings. Journal of the European Ceramic Society 24 (2004) Januar, S. 1-10

[252] Chen G.: Thermal Conductivity and Ballistic-Phonon Transport in the Cross-Plane Direction of Superlattices. Physical Review B 57 (1998) Juni, S. 14958-14973

[253] Brunel M., Enzo S., Jergel M., Luby S., Majkova E., Vavra I.: Structural Characterization and Thermal Stability of W/Si Multilayers. Journal of Materials Research 8 (1993) Oktober, S. 2600-2607

[254] Wen S.P., Zong R.L., Zeng F., Gu Y.L., Gao Y., Pan F.: Thermal Stability of Microstructure and Mechanical Properties of Ni/Ru Multilayers. Surface and Coatings Technology 202 (2008) Februar, S. 2040-2046

[255] Vassen Robert, Jarligo Maria O., Steinke Tanja, Mack Daniel E., Stöver Detlev: Overview on Advanced Thermal Barrier Coatings. Surface and Coatings Technology 205 (2010) November, S. 938-942

[256] Garg Jivtesh, Bonini Nicola, Kozinsky Boris, Marzari Nicola: Role of Disorder and Anharmonicity in the Thermal Conductivity of Silicon-Germanium Alloys: A FirstPrinciples Study. Physical Review Letters 106 (2011) Januar

[257] Terada By Y., Онкubo Kenji, Mohri Tetsuo: Thermal Conductivities of Platinum Alloys at High Temperatures. Platinum Metals Review 49 (2005) Januar, S. 21-26

[258] Konshunov V. A.: Determination of the Phonon Density of States from the Thermodynamic Functions of a Crystal: Nickel, Palladium, and Platinum. Soviet Physics Journal 22 (1979) August, S. 903-905

[259] Wang Y, Wang J J., Wang W Y., Mei Z G., Shang S L., Chen L Q., Liu Z K.: A MixedSpace Approach to First-Principles Calculations of Phonon Frequencies for Polar Materials. Journal of Physics: Condensed Matter 22 (2010) Mai, S. 202201

[260] Huebner Dennis, Rossner Christian, Vana Philipp: Light-Induced Self-Assembly of Gold Nanoparticles with a Photoresponsive Polymer Shell. Polymer (2016) Mai

[261] Assael M. J., Botsios S., Gialou K., Metaxa I. N.: Thermal Conductivity of Polymethyl Methacrylate (PMMA) and Borosilicate Crown Glass BK7. International Journal of Thermophysics 26 (2005) September, S. 1595-1605 
[262] Anderson D. R.: Thermal Conductivity of Polymers. Chemical Reviews 66 (1966) Dezember, S. 677-690

[263] Weber Erik H., Clingerman Matthew L., King Julia A.: Thermally Conductive Nylon 6,6 and Polycarbonate Based Resins. I. Synergistic Effects of Carbon Fillers. Journal of Applied Polymer Science 88 (2003) April, S. 112-122

[264] SÜSKE Erik, Scharf Thorsten, KREBs Hans-Ulrich, Junkers Thomas, BubACK Michael: Mechanism of Poly(methyl Methacrylate) Film Formation by Pulsed Laser Deposition. Journal of Applied Physics 100 (2006), S. 014906

[265] Nobile M. R., Amendola E., Nicolais L., Acierno D., Carfagna C.: Physical Properties of Blends of Polycarbonate and a Liquid Crystalline Copolyester. Polymer Engineering and Science 29 (1989) Februar, S. 244-257

[266] Nolle A. W.: Acoustic Determination of the Physical Constants of Rubber-Like Materials. The Journal of the Acoustical Society of America 19 (1947) Januar, S. 291-291 


\section{A. Author contributions}

This present thesis consists of the following four research articles of which the author of the thesis is the first author:

1. F. Döring, C. Eberl, S. Schlenkrich, F. Schlenkrich, S. Hoffmann, T. Liese, H. U. Krebs, S. Pisana, T. Santos, H. Schuhmann, M. Seibt, M. Mansurova, H. Ulrichs, V. Zbarsky and M. Münzenberg: Phonon localization in ultrathin layered structures. Applied Physics A 119:11-18 (2015) doi:10.1007/s00339-015-9037-z

2. F. Döring, H. Ulrichs, S. Pagel, M. Müller, M. Mansurova, M. Müller, C. Eberl, T. Erichsen, D. Huebner, P. Vana, K. Mann, M. Münzenberg and H. U. Krebs: Confinement of phonon propagation in laser deposited Tungsten/Polycarbonate multilayers.

New Journal of Physics 18, 092002, pp 1-9 (2016)

doi: 10.1088/1367-2630/18/9/092002

3. F. Döring and H. U. Krebs:

Thermal conductivity measurement by transient thermoreflectometry using highfluence excimer laser pulses at $248 \mathrm{~nm}$ wavelength.

Accepted for publication on 11 August 2016 for High Temperatures-High Pressures

4. F. Döring, A. Major, C Eberl and H. U. Krebs:

Minimized thermal conductivity in highly stable thermal barrier $\mathrm{W} / \mathrm{ZrO}_{2}$ multilayers.

Applied Physics A 122:872, pp 1-5 (2016)

doi:10.1007/s00339-016-0405-0 
All four articles were accepted in international, peer-reviewed journals and therefore fulfill the criteria for a cumulative $\mathrm{PhD}$ thesis according to the ProPhys program within the Georg-August University School of Science (GAUSS) program. While all of them show original research by the author, the individual contributions for each paper shall be clarified in the following.

Number one constitutes a co-work of many different groups, where samples with distinct thin film techniques were produced and investigated. Here, the author improved the PLD setup and hence contributed to fabrication of the PLD samples. Moreover, the author contributed to the interpretation and discussion of the measured data obtained by ultrafast pump-probe spectroscopy together with H. Ulrichs and M. Münzenberg. Finally, the author concepted, drafted and written the article with the help of H. U. Krebs and H. Ulrichs.

In the second article, the author conceived the idea of utilizing W/PC multilayers and using them as periodic and aperiodic nanostructures for phonon confinement under supervision of H. U. Krebs. The author improved the PLD setup in order to fabricate both, the very different materials $W$ and PC and combine them in new nanoscale multilayers with high interface density. All samples investigated in this chapter were fabricated and analyzed using x-ray reflectivity by the author, who contributed to the interpretation and discussion of all of the data presented in this paper. A part of the data was collected by S. Pagel, a Bachelor student supervised by the author. The manuscript was written and revised mainly by the author with the help of H. U. Krebs and H. Ulrichs.

Under advice of H. U. Krebs, the author conceived, implemented, tested and improved the new TTR method presented in the third paper. The author performed all steps from choosing the necessary hardware via setting up and adjusting the method up to data collection, analysis and interpretation. This paper was written by the author with the support of H. U. Krebs.

Paper number four again only shows work of the author with two small exceptions. Thermal stability x-ray measurements were performed by A. Major during her master's thesis, which was supervised by the author and figure 6.1 shows a $\mathrm{W} / \mathrm{ZrO}_{2}$ sample grown by $\mathrm{C}$. Eberl. Apart from this, sample fabrication, analysis, thermal conductivity measurements and data interpretation was done by the author. Also the manuscript was composed and revised by the author with the help of H. U. Krebs. 


\section{B. List of Publications}

1. F Döring, A Major, C Eberl, HU Krebs: Minimized thermal conductivity in highly stable thermal barrier W/ZrO2 multilayers. Applied Physics A 122:872 (2016)

2. F Döring, H Ulrichs, S Pagel, M Müller, M Mansurova, M Müller, C Eberl, T Erichsen, D Huebner, P Vana, K Mann, M Münzenberg, HU Krebs: Confinement of phonon propagation in laser deposited tungsten/polycarbonate multilayers. New Journal of Physics 18, 092002 (2016)

3. F Döring and HU Krebs: Thermal conductivity measurement by transient thermoreflectometry using high-fluence excimer laser pulses at $248 \mathrm{~nm}$ wavelength. High Temperatures High Pressures, accepted (2016)

4. F Döring, C Eberl, S Schlenkrich, F Schlenkrich, S Hoffmann, T Liese, HU Krebs, S Pisana, T Santos, H Schuhmann, M Seibt, M Mansurova, H Ulrichs, V Zbarsky, M Münzenberg: Phonon localization in ultrathin layered structures. Applied Physics A 119, 1 (2015)

5. F Döring, AL Robisch, C Eberl, M Osterhoff, A Ruhlandt, $T$ Liese, F Schlenkrich, S Hoffmann, M Bartels, T Salditt, HU Krebs: Sub-5 nm hard x-ray point focusing by a combined Kirkpatrick-Baez mirror and multilayer zone plate. Optics Express 21, 16 (2013)

6. H Ulrichs, D Meyer, M Müller, M Mansurova, F Döring: Numerical calculation of laser-induced thermal diffusion and elastic dynamics. AIP Conf. Proc. 1763, 040004 (2016)

7. C Klamt, A Dittrich, B Jaquet, C Eberl, F Döring, HU Krebs: Largest possible deviations from stoichiometry transfer during pulsed laser deposition. Applied Physics A 122, 7 (2016)

8. A Dittrich, C Eberl, S Schlenkrich, F Schlenkrich, F Döring, HU Krebs: Drastic deviations from stoichiometry transfer during pulsed laser deposition. Applied Physics A 122, 4 (2016) 
9. M Osterhoff, C Eberl, F Döring, RN Wilke, J Wallentin, HU Krebs, M Sprung, T Salditt: Towards multi-order hard X-ray imaging with multilayer zone plates. Journal of applied crystallography 48, 1 (2015)

10. C Eberl, M Osterhoff, F Döring, HU Krebs: MZP design and fabrication for efficient hard $x$-ray nano-focusing and imaging. SPIE Optical Engineering+ Applications 958808-958808-9 (2015)

11. M Osterhoff, F Döring, C Eberl, R Wilke, J Wallentin, HU Krebs, M Sprung, T Salditt: Progress on multi-order hard x-ray imaging with multilayer zone plates. SPIE Optical Engineering+ Applications 95920E-95920E-7 (2015)

12. C Eberl, F Döring, T Liese, F Schlenkrich, B Roos, M Hahn, T Hoinkes, A Rauschenbeutel, M Osterhoff, T Salditt, HU Krebs: Fabrication of laser deposited high-quality multilayer zone plates for hard X-ray nanofocusing. Applied Surface Science 307 (2014)

13. C Eberl, T Liese, F Schlenkrich, F Döring, H Hofsäss, HU Krebs: Enhanced resputtering and asymmetric interface mixing in W/Si multilayers. Applied Physics A 111, 2 (2013)

14. M Osterhoff, M Bartels, F Döring, C Eberl, $T$ Hoinkes, $S$ Hoffmann, T Liese, V Radisch, A Rauschenbeutel, AL Robisch, A Ruhlandt, F Schlenkrich, T Salditt, HU Krebs: Two-dimensional sub-5-nm hard x-ray focusing with MZP. SPIE Optical Engineering+ Applications 884802-884802-8 (2013) 


\section{Acknowledgments}

First and foremost I would like to thank my advisor Prof. Dr. Hans-Ulrich (Uli) Krebs. It has been an honor for me to be his last PhD student and I could not imagine a better working environment than the one he created for his group. I appreciate all of his fantastic supervision and his great contribution of time whenever it was needed as well as his support and advice that exceeded by far the technical realm.

Besides my advisor, I would like to thank Prof. Dr. Hans Hofsäss for being cosupervisor and second assessor of my thesis and also Prof. Cynthia Volkert, Prof. Dr. Philipp Vana, Prof. Dr. Michael Seibt and Prof. Dr. Vasily Moshnyaga for being members of my examination board.

For the fruitful collaborations of this work, I would like to thank all co-authors of the presented papers, especially Dr. Henning Ulrichs and Prof. Dr. Markus Münzenberg for their contribution with fs pump-probe measurements.

Furthermore, I would like to thank the whole PLD group for the wonderful atmosphere on the second floor: A special thanks goes to my office mate and close friend Dr. Christian (Karatepapayarennschnitzelbietz) Eberl, with whom I had a lot of nice moments in the office as well as during free time, e.g. when we saved the laser, traveled Göttingen, DPG-conferences, Ischia and the Harz, defeated the (M)FIB and so on and so on...

I also would like to thank the members of our neighbor office (sometimes referred to as the so-called "Hühnerstall"), namely Bea Jaquet and Christina Klamt, as well as the former members Sinja Pagel, Anna Major, Leonie Buntrock and Arne Dittrich. It was always a pleasure with you and thanks for the delicious cakes as well as the one or other kicker point. ;)

Likewise, I am grateful for the time I had together with all the former members of the PLD group, namely Dr. Susanne Schlenkrich, Anja Westphal, Sara-Lisa Jäckle, Dr. Sarah Hoffmann-Urlaub, Stephanie Demuth, Benedikt Ernst, Dr. Felix Schlenkrich and Dr. Tobias Liese and I am glad that within the PLD group colleagues became friends so easily. 
Apart from my scientific colleagues I would like to thank all the technicians who helped me during my doctorate. At first I would like to give thanks to Conni Mewes and Michael Malchow, who could help with many issues quickly and directly. Alike, I am glad for the support of the "E-Werkstatt", especially for the help of Michael Tetzlaff, who lend a hand to save our laser several times. Moreover, I would like to thank Frank Köhler from the fine mechanics workshop for his help with diverse sample holders and other setup items as well as Matthias Hahn and Volker Radisch for their support with FIB, SEM and TEM.

All in all, I really liked the productive environment in the whole Institute for Materials Physics, where I had a nice time. Therefore, I would like to thank all members of the institute.

Furthermore, I would like to thank the SFB 1073 as well as the sdw not only for financial support, because without that, this thesis would not be possible, but also for ideational support in several workshops and seminars that helped me to gain experience far from physics.

But apart from work, I had many friends from near and far, whom I would like to thank for enriching my life during my studies and my dissertation. Whosoever feels addressed here will surely remember the one or other funny anecdote. Last but not least, my strongest gratitude can only be expressed for the unconditional support I received from my parents Ursula and Ernst, my brother Ferdinand and my tremendously fantastic wife Christin. Thank you so very much. $\odot$ 